\title{
Uma prova funcional analítica da limitação uniforme de atratores para uma família de problemas parabólicos em $\mathbb{R}^{2}$
}

Bianca Paolini Lorenzi

\author{
DisSERTAÇÃO APRESENTADA \\ AO \\ Instituto de MATEMÁtica E EstatísticA \\ DA \\ Universidade de SÃo Paulo \\ PARA \\ OBTENÇÃO DO TÍTULO \\ $\mathrm{DE}$ \\ Mestre em CiênCias
}

Programa: Matemática

Orientador: Prof. Dr. Antônio Luiz Pereira

Durante o desenvolvimento deste trabalho a autora recebeu auxílio financeiro do CNPq 



\section{Uma prova funcional analítica da limitação uniforme de atratores para uma família de problemas parabólicos em $\mathbb{R}^{2}$}

Esta versão da dissertação contém as correções e alterações sugeridas pela Comissão Julgadora durante a defesa da versão original do trabalho, realizada em 22/09/2017. Uma cópia da versão original está disponível no Instituto de Matemática e Estatística da Universidade de São Paulo.

Comissão Julgadora:

- Prof. Dr. Antônio Luiz Pereira (orientador) - IME-USP

- Prof. Dra. Gleiciane da Silva Aragão - UNIFESP

- Prof. Dra. Pricila da Silva Barbosa - Externo 



\section{Agradecimentos}

Ao meu orientador Prof. Dr. Antônio Luiz Pereira, que vem me acompanhando desde a Iniciação Científica, com incansável atenção e dedicação, sempre me incentivando a ir além e a me aperfeiçoar.

Aos colegas e professores que assistiram minhas exposições nos Seminários de Equações de Evolução pelas valiosas contribuições.

À Ana Kelly de Oliveira e à Pricila da Silva Barbosa pelo desprendimento em me ajudar.

Aos meus pais pelo suporte, paciência e exemplo de força e luta.

Ao Marcelo Veronez Tola pelo grande companheirismo e amor.

À minha família pelo incentivo, em especial, ao meu tio José Geraldo Lúcio e à minha tia Maria Terêsa Rocha Triñanes. 


\section{Resumo}

Lorenzi, B. P. (2017). Uma prova funcional analítica da limitação uniforme de atratores para uma família de problemas parabólicos em $\mathbb{R}^{2}$ (Dissertação de Mestrado). Instituto de Matemática e Estatística, Universidade de São Paulo, São Paulo.

Este trabalho tem como principal objetivo estudar as constantes que aparecem em desigualdades relacionadas a operadores setoriais e suas potências fracionárias. Demonstramos que tais constantes dependem essencialmente do setor e da constante na desigualdade do resolvente associados ao operador. Como uma aplicação desses resultados, fornecemos uma prova alternativa para a limitação uniforme dos atratores de uma classe de problemas parabólicos semilineares obtidos por perturbação suave de um domínio.

Palavras-chave: Operadores setoriais. Equações parabólicas. Perturbação do domínio. Atrator global. Limitação dos atratores. 


\section{Abstract}

Lorenzi, B. P. (2017). An analytic functional proof of the uniform limitation of attractors for a family of parabolic problems in $\mathbb{R}^{2}$ (Dissertação de Mestrado). Instituto de Matemática e Estatística, Universidade de São Paulo, São Paulo.

This work has as main purpose to study the constants that appear in inequalities related to sectorial operators and their fractional powers. We show that these constants depend essentially on the sector and the constant in the resolvent inequality associated with the operator. As an application of these results, we provide an alternative proof for the uniform bound of the attractors of a class of semilinear parabolic problems obtained by smooth perturbation of a domain.

Keywords: Sectorial operators. Parabolic equations. Perturbation of the domain. Global attractor. Limitation of the attractors. 


\section{Sumário}

\begin{tabular}{ll}
\hline Introdução & 1
\end{tabular}

\begin{tabular}{lll}
\hline 1 & Operadores setoriais & 3
\end{tabular}

1.1 Operadores setoriais e semigrupos analíticos $\ldots \ldots \ldots \ldots$. . . . . . . 4

1.2 Potências fracionárias de operadores $\ldots \ldots \ldots \ldots \ldots$. . . . . . . . . . 24

1.3 Um teorema de imersão $\ldots \ldots \ldots \ldots \ldots \ldots$. . . . . . . . . . . . . 44

1.4 Uma importante aplicação $\ldots \ldots \ldots$. . . . . . . . . . . . . . . . . . . 46

2 Limitação uniforme dos atratores para uma família de perturbações suaves de um domínio $\quad 51$

2.1 Introdução: o problema e a redução a um domínio fixo . . . . . . . . . . . . . . . 51

2.2 Setorialidade dos operadores perturbados em vários espaços . . . . . . . . . . . . 52

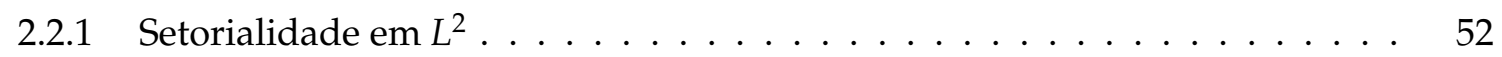

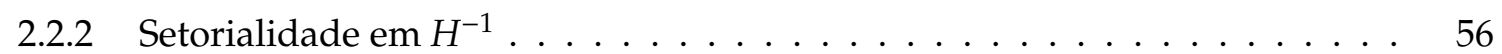

2.3 O problema abstrato em uma escala de espaços de Banach $\ldots \ldots \ldots \ldots$

2.4 Existência de soluções locais $\ldots \ldots \ldots \ldots$. . . . . . . . . . . . . . . . . 59

2.5 Funcional de Lyapunov e existência de solução global . . . . . . . . . . . . . . . . 65

2.6 Existência de um atrator global $\ldots \ldots \ldots \ldots$. . . . . . . . . . . . 69

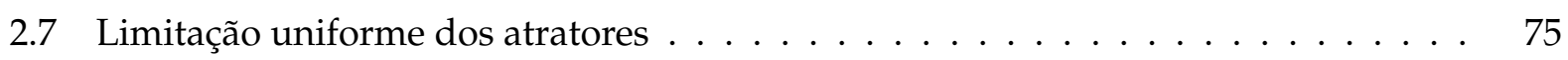

\begin{tabular}{lr}
\hline Referências Bibliográficas & 80
\end{tabular} 


\section{Introdução}

A motivação inicial desta monografia foi determinar exatamente do que dependem as constantes nas desigualdades envolvendo operadores setoriais e suas potências fracionárias. Um dos objetivos dessa análise seria obter resultados sobre a limitação uniforme de atratores de famílias de problemas parabólicos, fato essencial no estudo da continuidade dos atratores relativamente a perturbações no semigrupo. Sendo essa uma questão vital para a adequação da modelagem matemática de fenômenos utilizando semigrupos.

Para tanto, fizemos uma reescrita cuidadosa do primeiro capítulo de Henry (1981). Observamos que essas constantes dependem basicamente do setor e da constante na desigualdade do resolvente associados ao operador.

Resultou assim o primeiro capítulo desta dissertação no qual, definições, exemplos e as principais propriedades dos operadores setoriais e de suas potências fracionárias são abordados. Chamamos especial atenção do leitor para a importância do Teorema 1.3.4 em Henry (1981) nesse estudo. Este afirma que se $A$ é um operador setorial em um espaço de Banach $X$, então $-A$ é o gerador infinitesimal de um semigrupo analítico $\left\{e^{-A t}\right\}_{t \geq 0}$. As constantes que aparecem na sua demonstração são fundamentais para os nossos propósitos.

Finalizamos o primeiro capítulo com uma aplicação teórica dessa análise ao contexto dos problemas de Cauchy não lineares envolvendo operadores setoriais. Com base agora no terceiro capítulo de Henry (1981), mostramos, sob determinadas hipóteses, a limitação uniforme de um conjunto de atratores globais $\left\{\mathcal{A}_{\gamma}\right\}_{\gamma \in \Lambda}$ referentes aos problemas:

$$
\left\{\begin{array}{l}
\frac{d x}{d t}+A_{\gamma} x=f_{\gamma}(x), t>t_{0} \\
x\left(t_{0}\right)=x_{0} \in X_{\gamma}^{\alpha}
\end{array}\right.
$$

onde $\left\{A_{\gamma}\right\}_{\gamma \in \Lambda}$ é uma família de operadores setoriais em $X_{\gamma}^{\alpha}, 0 \leq \alpha<1$, com resolvente compacto, 
$D\left(A_{\gamma}\right) \supset D(A), \forall \gamma \in \Lambda$, sendo $\Lambda$ um espaço topológico e $A=A_{\gamma_{0}}, f_{\gamma}: U \rightarrow X, U$ um subconjunto aberto de $X_{\gamma}^{\alpha}$, é localmente Lipschitziana em $x$ e $f_{\gamma}$ aplica todos os subconjuntos fechados e limitados de $U$ em subconjuntos limitados de $X$.

Em um segundo momento, nos dedicamos a leitura de Barbosa, Pereira e Pereira (2016), o qual trata de uma família de problemas parabólicos semilineares obtidos por perturbações de classe $C^{1}$ do quadrado. A nossa principal meta era, usando a primeira parte do trabalho, obter uma limitação uniforme dos atratores em $L_{\infty}$.Dessa forma, torna-se possível, sem perda de generalidade, supor que as não linearidades são globalmente Lipschitzianas, em vez de adotar esta propriedade como hipótese adicional, como foi feito naquele artigo. Por razões técnicas, principalmente ligadas à comparação de normas em espaços fracionários, não conseguimos obter tal resultado na generalidade pretendida.

Partimos para o estudo do mesmo problema no caso mais simples de domínios suaves discutido em Pereira e Pereira (2007). O objetivo do segundo capítulo então é o de provar a limitação uniforme dos atratores para uma família de perturbações suficientemente regulares de $\Omega$, um aberto limitado do $\mathbb{R}^{2}$ com fronteira suave, segundo um viés funcional analítico. Arrieta, Carvalho e Rodríguez-Bernal (2000) demonstram a limitação uniforme dos atratores através de técnicas de comparação.

Destacamos um resultado que foi essencial para essa conclusão, o de podermos supor que toda perturbação $h$, com $h$ um difeomorfismo de ordem 3, é tal que a "normal perturbada"coincide com a normal usual desde que exijamos um pouco mais de regularidade do domínio e $\left\|h-i_{\Omega}\right\|_{\mathcal{C}^{3}\left(\Omega, \mathbb{R}^{2}\right)}$ seja pequena, onde $i_{\Omega}$ denota a identidade em $\Omega$.

Futuramente, gostaríamos de tentar aplicar essa abordagem para o mesmo problema em uma dimensão maior. As desigualdades de Nirenberg - Gagliardo, que garantem a inclusão dos espaços de potências fracionárias em subespaços de $L_{\infty}$ sob determinadas condições, parecem indicar que é necessário estudar o problema nos espaços $L_{p}$. 


\section{Capítulo 1}

\section{Operadores setoriais}

Neste capítulo, nas seções um, dois e três, realizamos um estudo minucioso sobre os operadores setoriais e suas potências fracionárias com base em Henry (1981) a fim de analisarmos a dependência das constantes que aparecem em desigualdades envolvendo tais operadores. Na quarta e última seção, aplicamos esse estudo para obter resultados importantes no contexto de problemas de Cauchy não lineares.

Utilizaremos as seguintes notações, sendo $\lambda \in \mathbb{C}, X$ um espaço normado, $A$ um operador linear e $v \geq 0$,

$\mathfrak{R}(\lambda)=$ parte real de $\lambda$;

$\mathfrak{J}(\lambda)=$ parte imaginária de $\lambda$;

$L(X)=$ espaço dos operadores lineares contínuos com domínio $X$ e imagem em $X$;

$D(A)=$ domínio de $A$;

$R(A)=$ imagem $\operatorname{de} A ;$

$\rho(A)=$ conjunto resolvente $\operatorname{de} A$;

$\sigma(A)=\operatorname{espectro} \operatorname{de} A ;$

$C^{v}(S, X)=$ espaço das funções $[v]$ vezes continuamente diferenciáveis de $S$, um subconjunto aberto de um espaço de Banach, em outro espaço de Banach $X$, onde a derivada de ordem [ $v$ ] satisfaz a condição de Holder com expoente $v-[v]$, se $v$ não é inteiro. A norma é dada por

$$
\|f\|_{C^{v}(S, X)}=\sum_{k=0}^{[v]} \sup _{S}\left\|D^{k} f\right\|+\sup _{x \neq y} \frac{\left\|D^{[v]} f(x)-D^{[v]} f(y)\right\|}{\|x-y\|^{\alpha}}
$$


$\operatorname{com} \alpha=v-[v]$ e o último termo é omitido quando $v$ é inteiro.

$C_{c}^{k}(S, X)=$ subespaço linear das funções em $C^{k}(S, X)$ que possuem suporte compacto em $S$ (isto é, $f(x)=0, \forall x \in S \backslash K$, com $K$ um compacto).

$L_{p}(\Omega, X)=$ espaços das funções $f$, de um espaço de medida $\Omega$ em um espaço de Banach $X$, mensuráveis com $|f|^{p}$ integrável,

$$
\|f\|_{L_{p}(\Omega, X)}=\left\{\int_{\Omega}\|f(t)\|_{X}^{p} d t\right\}^{\frac{1}{p}}
$$

para $1 \leq p<\infty$.

$L_{\infty}(\Omega, X)=$ espaço das funções essencialmente limitadas de $\Omega$ em $X$,

$$
\|f\|_{L_{\infty}(\Omega, X)}=\sup \operatorname{ess}\left\{\|f(t)\|_{X} \mid t \in \Omega\right\} .
$$

$W^{k, p}(\Omega, X)=$ espaço de Sobolev das funções $f \in L_{p}(\Omega, X)$ que possuem derivadas distribucionais de ordem $\leq k$ todas em $L_{p}$ (aqui $\Omega$ é um aberto do $\mathbb{R}^{n}$ ).

$$
\|f\|_{W^{k, p}(\Omega, X)}=\left\{\int_{\Omega} \sum_{j=0}^{k}\left\|f^{j}(t)\right\|^{p} d t\right\}^{\frac{1}{p}} .
$$

$H^{k}(\Omega, X)=W^{k, 2}(\Omega, X)$, que é um espaço de Hilbert quando X é um espaço de Hilbert.

\subsection{Operadores setoriais e semigrupos analíticos}

Nesta seção, apresentamos o conceito de setorialidade, exibimos alguns exemplos de operadores com tal propriedade e, por fim, estudamos o semigrupo analítico relacionado a este tipo de operador através de um resultado que será central em nosso trabalho.

Definição 1.1.1 Um operador linear A em um espaço de Banach X é dito um operador setorial se é um operador densamente definido, fechado e tal que para algum $\phi \in\left(0, \frac{\pi}{2}\right), M \geq 1$ e algum número real a, o setor:

$$
S_{a, \phi}=\{\lambda \in \mathbb{C}|\phi \leq| \arg (\lambda-a) \mid \leq \pi, \lambda \neq a\}
$$

está contido no resolvente de A e vale a desigualdade

$$
\left\|(\lambda I-A)^{-1}\right\| \leq \frac{M}{|\lambda-a|}, \forall \lambda \in S_{a, \phi}
$$




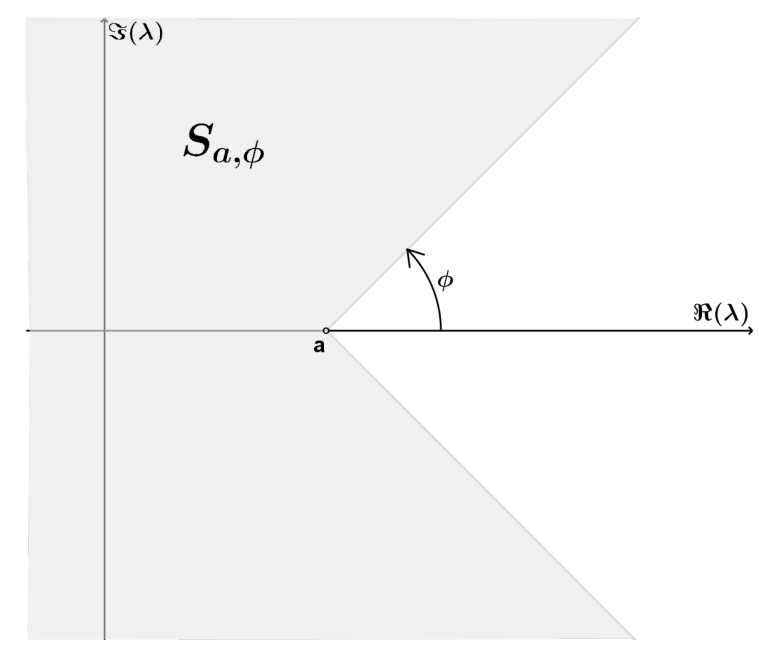

Figura 1.1: Setor $S_{a, \phi}$.

Vejamos a seguir alguns exemplos de operadores setoriais.

Exemplo 1.1.2 Seja A um operador linear limitado em um espaço de Banach X. Então, A é setorial.

De fato, como $A$ é limitado $(D(A)=X)$, temos que $A$ é densamente definido e fechado. Quanto ao setor para o operador $A$ e a correspondente desigualdade, precisaremos fazer alguns cálculos a fim de determiná-los:

i) Observamos que se $\lambda \in \mathbb{C}$ é tal que $|\lambda|>\|A\|$, então $\forall x \in X$,

$$
\begin{aligned}
\|(\lambda I-A) x\| & \geq\|\lambda|\|x\|-\|A x\|| \\
& \geq\|\lambda|\|x\|-\|A\|\|x\|| \\
& \geq(|\lambda|-\|A\|)\|x\|,
\end{aligned}
$$

o que signifca que o conjunto $\{\lambda \in \mathbb{C}\|\lambda \mid>\| A \|\} \subset \rho(A)$. Dessa forma, basta considerarmos, por exemplo, o vértice $a=-2\|A\|$ e o ângulo $\phi=\frac{\pi}{4}$ para obter um setor contido no $\rho(A)$, conforme Figura 1.2 


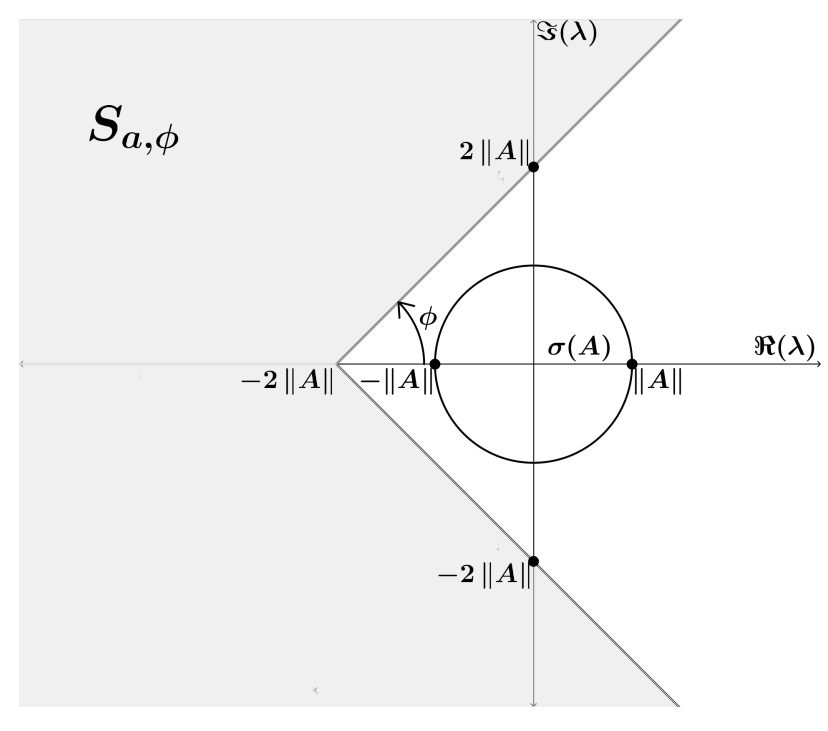

Figura 1.2: Possível setor para A.

ii) Para $\lambda$ tal como em i), podemos escrever o operador resolvente $(\lambda I-A)^{-1}$ como a série $\sum_{k=0}^{\infty} \frac{A^{k}}{\lambda^{k+1}}$. Isso porque, denotando $S_{n}$ a sua soma parcial $\sum_{k=0}^{n} \frac{A^{k}}{\lambda^{k+1}}$, se $n>m$ :

$$
\begin{aligned}
\left\|S_{n}-S_{m}\right\| & =\left\|\frac{A^{m+1}}{\lambda^{m+2}}+\cdots+\frac{A^{n}}{\lambda^{n+1}}\right\| \\
& \leq \frac{\|A\|}{|\lambda|}\left(\frac{\|A\|^{m}}{|\lambda|^{m+1}}+\cdots+\frac{\|A\|^{n-1}}{|\lambda|^{n}}\right),
\end{aligned}
$$

o que nos mostra que $S_{n}$ é uma sequência de Cauchy em $L(X)$ afinal, $\frac{\|A\|}{|\lambda|}<1$. Como $X$ é um espaço de Banach, $L(X)$ é completo, donde $S_{n}$ converge para algum operador $S \in L(X)$.

Agora, $(\lambda I-A) S_{n}=S_{n}(\lambda I-A)=I-\frac{A^{n}}{\lambda^{n}}$ e, essa expressão converge para $(\lambda I-A) S=S(\lambda I-A)=I$. Logo, $(\lambda I-A)^{-1}=\sum_{k=0}^{\infty} \frac{A^{k}}{\lambda^{k+1}}$.

iii) Notemos que para qualquer $\lambda \in S_{a, \phi}$, vale que $|\lambda| \geq \sqrt{2}\|A\|$ (trata-se da altura $\mathrm{h}$ do triângulo isóceles com os lados iguais medindo $2\|A\|$ conforme Figura 1.3), daí juntando essas considerações, encontramos:

$$
\begin{aligned}
\left\|\lambda(\lambda I-A)^{-1}\right\| & \leq|\lambda| \sum_{n=0}^{\infty} \frac{\|A\|^{n}}{|\lambda|^{n+1}} \\
& =\sum_{n=0}^{\infty}\left(\frac{\|A\|}{|\lambda|}\right)^{n} \\
& \leq \sum_{n=0}^{\infty}\left(\frac{\|A\|}{|\sqrt{2}\|A\||}\right)^{n} \\
& =\sum_{n=0}^{\infty}\left(\frac{1}{\sqrt{2}}\right)^{n} \\
& =2+\sqrt{2},
\end{aligned}
$$


ou seja, $\left\|(\lambda I-A)^{-1}\right\| \leq \frac{2+\sqrt{2}}{|\lambda|}$, para $\forall \lambda \in S_{a, \phi}$.

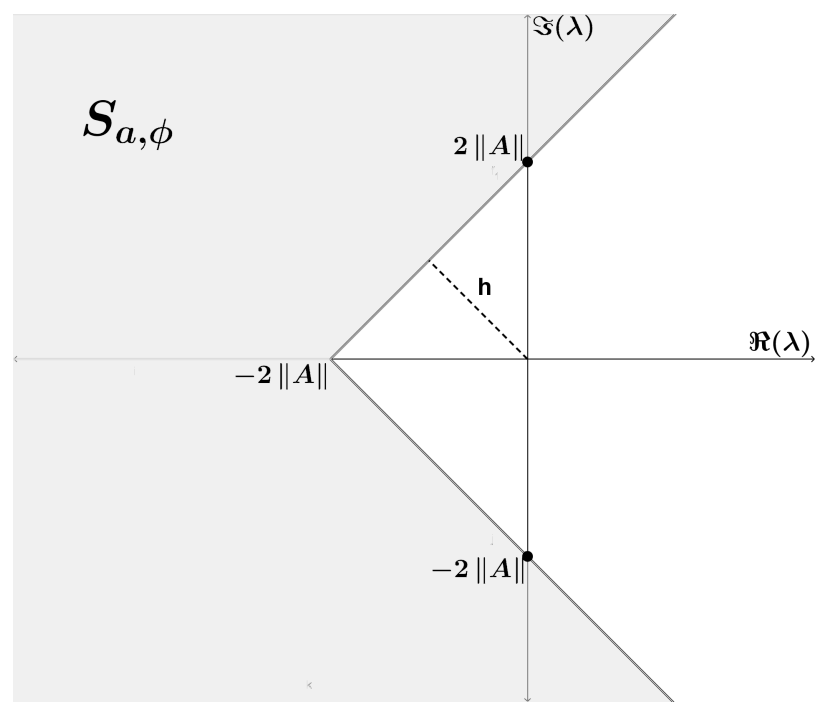

Figura 1.3: Setor $S_{a, \phi}$.

iv) Neste ponto, devemos adequar esta última desigualdade nos moldes daquela presente na Definição 1.1.1. Como para todo $\lambda \in S_{a, \phi}$, temos $|\lambda| \geq \sqrt{2}\|A\|$, segue que:

$$
\begin{aligned}
\frac{|\lambda-(-2\|A\|)|}{|\lambda|} & \leq\left|1+\frac{2\|A\|}{\lambda}\right| \\
& \leq 1+\frac{2\|A\|}{|\lambda|} \\
& \leq 1+\frac{2}{\sqrt{2}} \\
& =1+\sqrt{2} .
\end{aligned}
$$

Portanto,

$$
\left\|(\lambda I-A)^{-1}\right\| \leq \frac{(2+\sqrt{2})(1+\sqrt{2})}{|\lambda-(-2\|A\|)|}, \forall \lambda \in S_{a, \phi} .
$$

E, terminamos a demonstração de que o operador $A$ é setorial.

Exemplo 1.1.3 Seja A um operador densamente definido em um espaço de Hilbert $(H,<,>)$, limitado inferiormente e autoadjunto. Então, A é setorial.

Com efeito, sendo $A$ um operador densamente definido e autoadjunto, $A$ é fechado e seu espectro $\sigma(A)$ é um subconjunto do eixo real. Além disso, como $A$ é limitado inferiormente, isto é, existe $a \in \mathbb{R}$ tal que $<A x, x>\geq a\|x\|^{2}, \forall x \in D(A), \sigma(A)$ deve estar contido no intervalo $[a, \infty)$.

Assim, o setor $S_{a, \frac{\pi}{4}}=\left\{\lambda \in \mathbb{C}\left|\frac{\pi}{4} \leq\right| \arg (\lambda-a) \mid \leq \pi, \lambda \neq a\right\}$ está contido no resolvente de $A$, vide 
Figura 1.4 Resta então obtermos uma desigualdade como na Definição 1.1.1

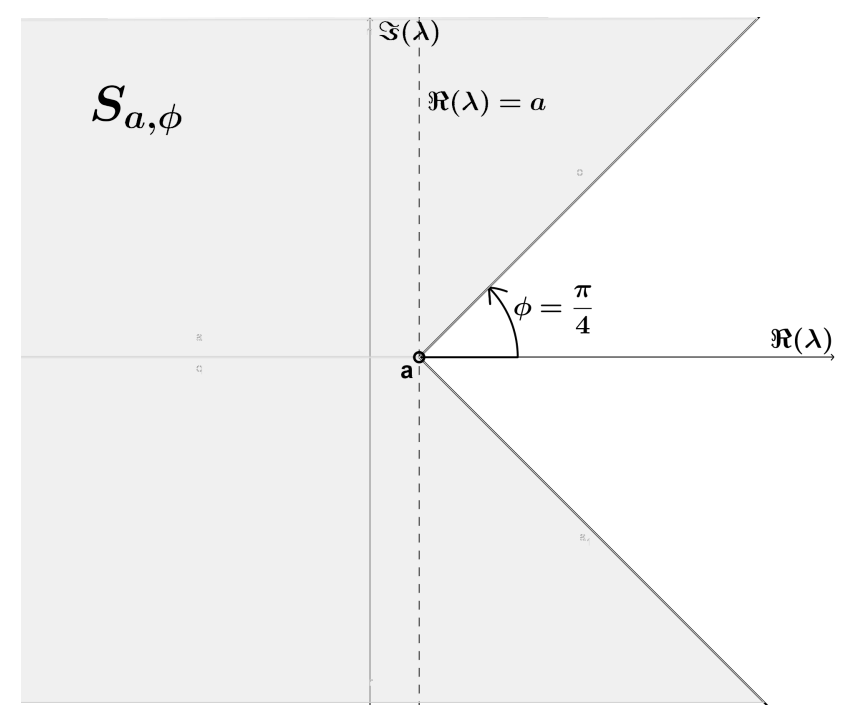

Figura 1.4: Possível setor para A.

Sejam $\lambda \in S_{a, \frac{\pi}{4}}$ e $\tilde{\lambda}=\lambda-a$. Notemos que $\mathfrak{R}(\lambda)<a$ implica $\mathfrak{R}(\tilde{\lambda})<0$ enquanto que $\mathfrak{R}(\lambda) \geq a$, $|\mathfrak{J}(\tilde{\lambda})| \geq|\mathfrak{R}(\tilde{\lambda})|$. Estudemos a seguir cada uma dessas possibilidades:

Caso 1: $\lambda=\tilde{\lambda}+a \operatorname{com} \Re(\tilde{\lambda})<0$.

$$
\begin{aligned}
\|(\lambda I-A) x\|^{2} & =\|(\tilde{\lambda} I-(A-a)) x\|^{2} \\
& =|\tilde{\lambda}|^{2}\|x\|^{2}+\|(A-a) x\|^{2}-2 \mathfrak{R}(<(A-a) x, \tilde{\lambda} x>) \\
& =|\tilde{\lambda}|^{2}\|x\|^{2}+\|(A-a) x\|^{2}-2 \mathfrak{R}(\tilde{\lambda})<(A-a) x, x> \\
& \geq|\tilde{\lambda}|^{2}\|x\|^{2},
\end{aligned}
$$

donde $\|(\lambda I-A)\| \geq|\tilde{\lambda}| \mathrm{e}$, consequentemente, $\left\|(\lambda I-A)^{-1}\right\| \leq \frac{1}{|\lambda-a|}$.

Caso $2: \lambda=\tilde{\lambda}+a \operatorname{com}|\mathfrak{J}(\tilde{\lambda})| \geq|\mathfrak{R}(\tilde{\lambda})|$.

$$
\begin{aligned}
\|(\lambda I-A) x\|^{2} & =\|(\tilde{\lambda} I-(A-a)) x\|^{2} \\
& =|\mathfrak{J}(\tilde{\lambda})|^{2}\|x\|^{2}+\|(\mathfrak{R}(\tilde{\lambda})-(A-a)) x\|^{2} \\
& \geq|\mathfrak{J}(\tilde{\lambda})|^{2}\|x\|^{2} \\
& \geq \frac{|\tilde{\lambda}|^{2}}{2}\|x\|^{2},
\end{aligned}
$$

onde usamos que $|\mathfrak{J}(\tilde{\lambda})| \geq|\mathfrak{R}(\tilde{\lambda})|$ implica $|\tilde{\lambda}|^{2}=\mathfrak{R}(\tilde{\lambda})^{2}+\mathfrak{J}(\tilde{\lambda})^{2} \leq 2 \mathfrak{J}(\tilde{\lambda})^{2}$. Segue que $\|(\lambda I-A)\|$ $\geq \frac{|\tilde{\lambda}|}{\sqrt{2}}$, ou ainda, $\left\|(\lambda I-A)^{-1}\right\| \leq \frac{\sqrt{2}}{|\lambda-a|}$. 
Finalmente, reunindo essas considerações, obtemos $\left\|(\lambda I-A)^{-1}\right\| \leq \frac{\sqrt{2}}{|\lambda-a|} ; \forall \lambda \in S_{a, \frac{\pi}{4}}$, o que conclui a prova de que o operador $A$ é setorial.

Exemplo 1.1.4 Suponha que A é um operador setorial em um espaço de Banach X e $\left\|A(\lambda I-A)^{-1}\right\| \leq C$ para $|\arg (\lambda)| \geq \phi_{0},|\lambda| \geq R_{0}$ com $C, R_{0}$ constantes positivas e $\phi_{0}<\frac{\pi}{2}$. Se B é um operador linear fechado tal que $D(B) \supset D(A) e,\|B x\| \leq \varepsilon\|A x\|+K\|x\| ; \forall x \in D(A)$, onde $K, \varepsilon$ são constantes positivas com $\varepsilon C<1$, então, $A+B$ é setorial.

Como $\overline{D(A)}=X$ e $D(B) \supset D(A)$, temos que $\overline{D(A+B)}=\overline{D(A)}=X \mathrm{e}, A+B$ é densamente definido. Além disso, $A+B$ é fechado.

De $\left\|A(\lambda I-A)^{-1}\right\| \leq C$ em $S=\left\{\lambda \in \mathbb{C}|| \arg (\lambda)\left|\geq \phi_{0},\right| \lambda \mid \geq R_{0}\right\}$, seguem os cálculos:

$$
\begin{aligned}
C & \geq\left\|(-(\lambda I-A)+\lambda I)(\lambda I-A)^{-1}\right\| \\
& \geq\left|\left\|\lambda(\lambda I-A)^{-1}\right\|-\|I\|\right| \\
& \geq|\lambda|\left\|(\lambda I-A)^{-1}\right\|-1,
\end{aligned}
$$

ou seja, $\left\|(\lambda I-A)^{-1}\right\| \leq \frac{C+1}{|\lambda|}$ para qualquer $\lambda$ nessa região.

Ainda, aplicando esta última desigualdade $\operatorname{com} \lambda \in S, x \in X$ :

$$
\begin{aligned}
\left\|B(\lambda I-A)^{-1} x\right\| & \leq \varepsilon\left\|A(\lambda I-A)^{-1} x\right\|+K\left\|(\lambda I-A)^{-1} x\right\| \\
& \leq \varepsilon C\|x\|+K \frac{C+1}{|\lambda|}\|x\| .
\end{aligned}
$$

Portanto, $\left\|B(\lambda I-A)^{-1}\right\| \leq \varepsilon C+K \frac{C+1}{|\lambda|}, \lambda \in S$.

A partir dessas considerações, podemos estimar $\left\|(\lambda I-(A+B))^{-1}\right\|$ :

$$
\begin{aligned}
\left\|(\lambda I-(A+B))^{-1}\right\| & =\left\|\left((\lambda I-A)\left(I-B(\lambda I-A)^{-1}\right)\right)^{-1}\right\| \\
& \leq\left\|(\lambda I-A)^{-1}\right\|\left\|\left(I-B(\lambda I-A)^{-1}\right)^{-1}\right\| \\
& \leq \frac{C+1}{|\lambda|}\left\|\left(I-B(\lambda I-A)^{-1}\right)^{-1}\right\| \\
& \leq \frac{C+1}{|\lambda|}\left(1-\varepsilon C-K \frac{C+1}{|\lambda|}\right)^{-1} \\
& \leq \frac{\text { Constante }}{|\lambda|},
\end{aligned}
$$

onde usamos que se $T=I-B(\lambda I-A)^{-1}$, então para $|\lambda|$ suficientemente grande de forma que $\varepsilon C+K \frac{C+1}{|\lambda|}<1$, resulta $\|T-I\|<1$ e, consequentemente, podemos escrever $T^{-1}=\sum_{n=0}^{\infty}(I-T)^{n}$ donde, $\left\|T^{-1}\right\| \leq \frac{1}{1-\|I-T\|}$. Note que nessa passagem foi essencial que $\varepsilon C<1$.

Obtivemos assim $\left\|(\lambda I-(A+B))^{-1}\right\| \leq \frac{\text { Constante }}{|\lambda|}$, para $\lambda \in \mathbb{C} \operatorname{com}|\arg (\lambda)| \geq \phi_{0}$ e $|\lambda|$ grande o 
suficiente para que $\varepsilon C+K \frac{C+1}{|\lambda|}<1$. Mas, ainda não temos exatamente um setor como na Definição 1.1.1.

Agora, $\varepsilon C+K \frac{C+1}{|\lambda|}<1 \Leftrightarrow|\lambda|>\frac{K(C+1)}{1-\varepsilon C}$. Daí, supondo que $R_{0}<\frac{K(C+1)}{1-\varepsilon C}$, um possível setor poderia ser com vértice $a=-\frac{K(C+1)}{1-\varepsilon C}-\delta, \delta>0$ e ângulo $\phi \geq \phi_{0}$ tal que os semi-eixos não interceptem a circunferência $|\lambda|=\frac{K(C+1)}{1-\varepsilon C}$, conforme Figura 1.5 . E, vale que:

$$
\left\|(\lambda I-(A+B))^{-1}\right\| \leq \frac{\text { Constante }}{|\lambda|} \leq \frac{\text { Constante }}{|\lambda-a|}, \forall \lambda \in S_{a, \phi} .
$$

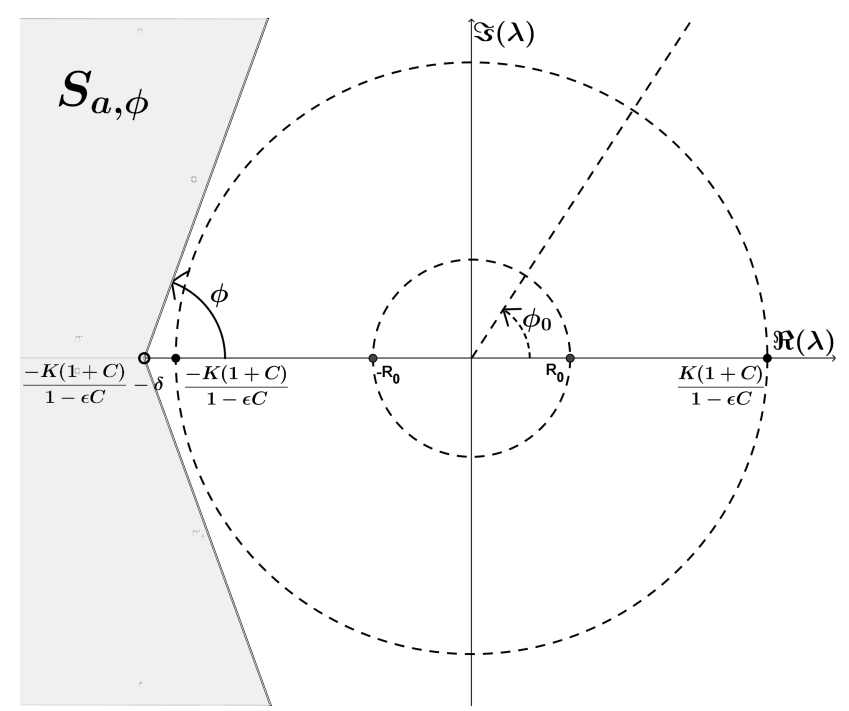

Figura 1.5: Possível setor para A.

Vale observar que uma outra possibilidade seria transladarmos o vértice $a$ do setor para a esquerda, obtendo $a^{\prime} \operatorname{com} a^{\prime}<a$, o que permitiria tomar um ângulo $\phi^{\prime}$ tal que $\phi^{\prime}<\phi$ ainda de forma a garantir a não interseção dos semi-eixos com a circunferência $|\lambda|=\frac{K(C+1)}{1-\varepsilon C}$.

Finalizamos dessa maneira a prova de que $A+B$ é setorial nas condições descritas acima.

Exemplo 1.1.5 Sejam A um operador setorial em X e B um operador setorial em $Y$, com X e $Y$ espaços de Banach. Então, $A \times B$ é setorial em $X \times Y$, onde $(A \times B)(x, y)=(A x, B y)$, para $x \in D(A)$ e $y \in D(B)$.

Consideremos o espaço produto $X \times Y$ equipado com a norma $\|(x, y)\|=\operatorname{máx}\left\{\|x\|_{X},\|y\|_{Y}\right\}$, sendo $\|\cdot\|_{X}$ e $\|\cdot\|_{Y}$ as normas em $X$ e $Y$ respectivamente.

Denotemos o setor correspondente ao operador A por

$$
S_{a, \phi}=\{\lambda \in \mathbb{C}|\phi \leq| \arg (\lambda-a) \mid \leq \pi, \lambda \neq a\} \subset \rho(A), a \in \mathbb{R}, \phi \in\left(0, \frac{\pi}{2}\right)
$$

e,

$$
\left\|\left(\lambda I_{X}-A\right)^{-1}\right\| \leq \frac{M_{A}}{|\lambda-a|}, \forall \lambda \in S_{a, \phi}, M_{A} \geq 1,
$$


onde $I_{X}$ é a identidade em $X$. E, para o operador B,

$$
S_{b, \psi}=\{\lambda \in \mathbb{C}|\psi \leq| \arg (\lambda-b) \mid \leq \pi, \lambda \neq b\} \subset \rho(B), b \in \mathbb{R}, \psi \in\left(0, \frac{\pi}{2}\right)
$$

e,

$$
\left\|\left(\lambda I_{Y}-B\right)^{-1}\right\| \leq \frac{M_{B}}{|\lambda-b|}, \forall \lambda \in S_{b, \psi}, M_{B} \geq 1
$$

sendo $I_{Y}$ a identidade em $Y$.

É imediato que o operador $A \times B$ é densamente definido e fechado.

Observamos que para podermos calcular, por exemplo, $\left\|\left(\lambda\left(I_{X} \times I_{Y}\right)-(A \times B)\right)^{-1}\right\|=\|\left(\left(\lambda I_{X}-\right.\right.$ $\left.A) \times\left(\lambda I_{Y}-B\right)\right)^{-1} \|, \lambda$ deve ser tal que ambos os operadores $\left(\lambda I_{X}-A\right)^{-1}$ e $\left(\lambda I_{Y}-B\right)^{-1}$ existem e são limitados. Dessa forma, precisamos determinar um setor comum para A e B.

Analisando as relações possíveis entre os vértices dos setores, $a e b$, e entre seus ângulos, $\phi e \psi$, como ilustram as Figuras 1.6 e 1.7, vemos que um setor comum é $S_{c, \theta}$, onde $c=\min \{a, b\}$ e, $\theta=\operatorname{máx}\{\phi, \psi\}$.

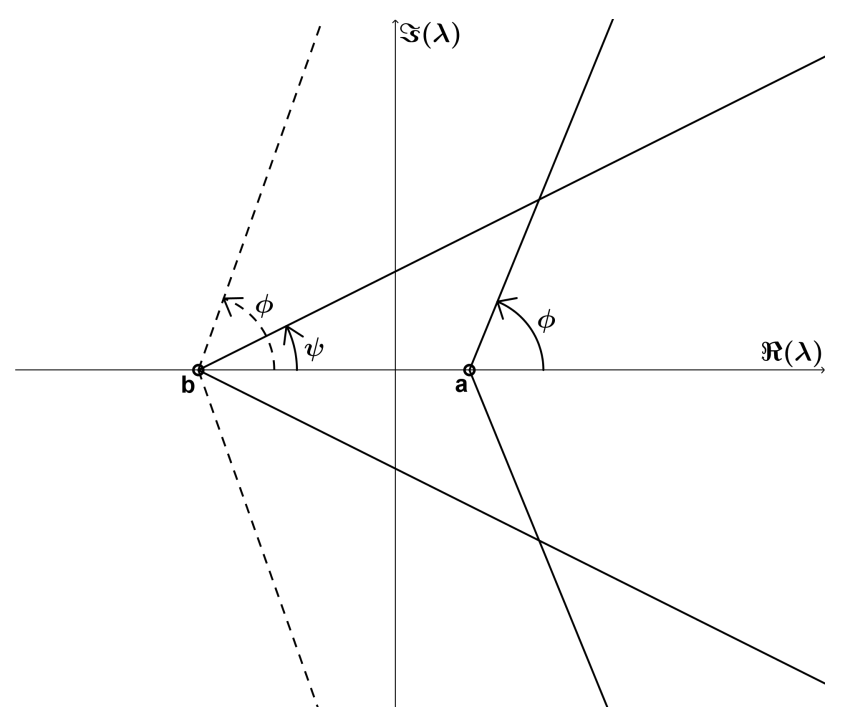

Figura 1.6: Possível situação em que $b<a$ e $\psi<\phi$. 


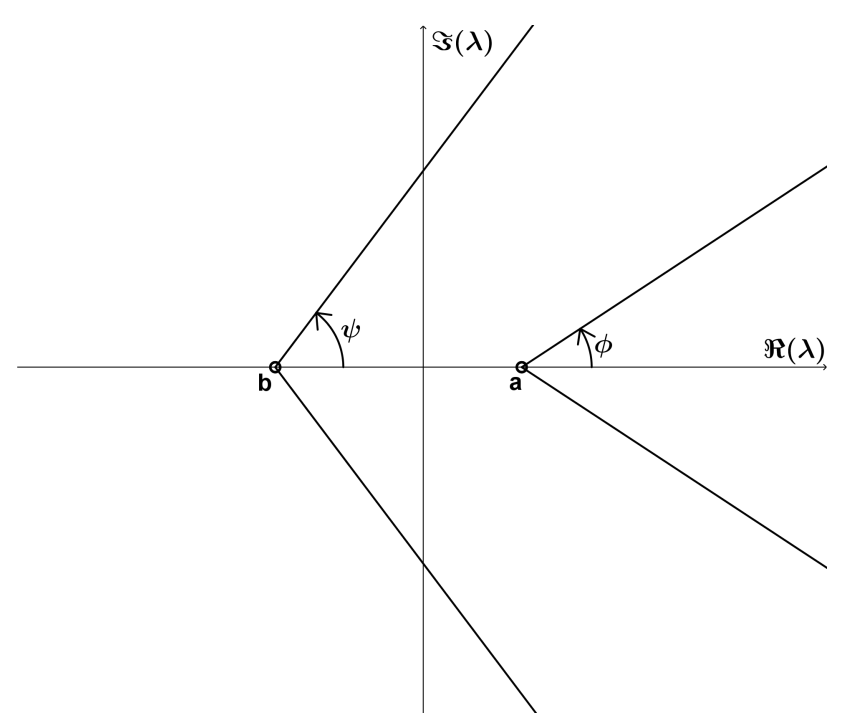

Figura 1.7: Possível situação em que $b<a$ e $\phi<\psi$.

Por fim, resta obtermos uma desigualdade como na Definição 1.1.1. Para $\lambda \in S_{c, \theta}$ e $\|(x, y)\|=1$, temos:

$$
\begin{aligned}
\left\|\left(\lambda\left(I_{X} \times I_{Y}\right)-(A \times B)\right)^{-1}(x, y)\right\| & =\left\|\left(\left(\lambda I_{X}-A\right)^{-1} \times\left(\lambda I_{Y}-B\right)^{-1}\right)(x, y)\right\| \\
& =\operatorname{máx}\left\{\left\|\left(\lambda I_{X}-A\right)^{-1} x\right\|_{X}\left\|\left(\lambda I_{Y}-B\right)^{-1} y\right\|_{Y}\right\} \\
& \leq \operatorname{máx}\left\{\frac{M_{A}\|x\|_{X}}{|\lambda-a|}, \frac{M_{B}\|y\|_{Y}}{|\lambda-b|}\right\} \\
& \leq \operatorname{máx}\left\{\frac{M_{A}}{|\lambda-a|}, \frac{M_{B}}{|\lambda-b|}\right\} .
\end{aligned}
$$

E então, supondo $a<b$, segue que $|\lambda-b| \geq K|\lambda-a|, \forall \lambda \in S_{c, \theta}$, sendo $K$ uma constante. Aplicando isso na desigualdade (1.1), encontramos:

$$
\begin{aligned}
\left\|\left(\lambda\left(I_{X} \times I_{Y}\right)-(A \times B)\right)^{-1}(x, y)\right\| & \leq \operatorname{máx}\left\{\frac{M_{A}}{|\lambda-a|}, \frac{M_{B}}{K|\lambda-a|}\right\} \\
& =\operatorname{máx}\left\{M_{A}, \frac{M_{B}}{K}\right\} \frac{1}{|\lambda-a|} \\
& =\frac{\text { Constante }}{|\lambda-c|} .
\end{aligned}
$$

O caso $b<a$ é análogo.

Logo, provamos que o produto de dois operadores setoriais é setorial.

Definição 1.1.6 Um semigrupo analítico em um espaço de Banach X é uma família de operadores lineares continuos em $X,\{T(t)\}_{t \geq 0}$, satisfazendo: 
(i) $T(0)=I, T(t) \circ T(s)=T(t+s)$, para $t \geq 0$ e $s \geq 0$,

(ii) $T(t) x \rightarrow x$ quando $t \rightarrow 0^{+}$, para cada $x \in X$,

(iii) A aplicação $t \rightarrow T(t) x$ é real analítica em $0<t<\infty$, para cada $x \in X$.

Definição 1.1.7 O gerador infinitesimal L deste semigrupo é definido por

$$
L x=\lim _{t \rightarrow 0^{+}} \frac{1}{t}(T(t) x-x),
$$

sendo $D(L)$ o conjunto dos $x \in X$ para os quais tal limite existe em $X$.

Observação 1.1.8 É usual denotar $T(t)=e^{L t}$.

Teorema 1.1.9 Se A é um operador setorial, então - A é o gerador infinitesimal de um semigrupo analítico $\left\{e^{-A t}\right\}_{t \geq 0}$, onde

$$
e^{-A t}=\frac{1}{2 \pi i} \int_{\Gamma}(\lambda I+A)^{-1} e^{\lambda t} d \lambda
$$

sendo $\Gamma$ um contorno em $\rho(-A) \operatorname{com} \arg (\lambda) \rightarrow \pm \theta$ quando $|\lambda| \rightarrow \infty$ para algum $\theta \in\left(\frac{\pi}{2}, \pi\right)$.

Além disso, $e^{-A t}$ pode ser estendido analiticamente a um setor $\{t \in \mathbb{C} \backslash\{0\}|| \arg (t) \mid<\epsilon\}$ contendo o eixo real positivo e se $\mathfrak{R}(\sigma(A))>a$, isto é, $\mathfrak{R}(\lambda)>$ a sempre que $\lambda \in \sigma(A)$, então para $t>0$ :

$$
\left\|e^{-A t}\right\| \leq C_{1} e^{-a t} \text { e }\left\|A e^{-A t}\right\| \leq \frac{C_{2}}{t} e^{-a t},
$$

sendo $C_{1}>0$ e $C_{2}>0$ dadas por

$$
\begin{aligned}
& C_{1}=\frac{M}{2 \pi} \int_{\Gamma}\left|e^{\mu}\right| \frac{|d \mu|}{|\mu|} e, \\
& C_{2}=\frac{1+M}{2 \pi} \int_{\Gamma}\left|e^{\mu}\right||d \mu|,
\end{aligned}
$$

onde Mé a constante que aparece na Definição 1.1.1.

Finalmente, $\frac{d}{d t} e^{-A t}=-A e^{-A t}$ para $t>0$.

A demonstração desse Teorema depende do seguinte resultado auxiliar:

Lema 1.1.10 Sejam $\Gamma$ como no Teorema 1.1.9. $\Gamma^{\prime}$ um pequeno deslocamento de $\Gamma$ para a direita e a reta $R: x_{0}+i s, s \in[-y, y]$, onde $x_{0}<0 e|y|=-\tan (\theta)\left|x_{0}\right|$, de modo a fechar o contorno $\Gamma$ (vide Figura 1.8). Se $\mu \in \Gamma^{\prime}$, então,

$$
\int_{R} \frac{e^{\lambda t}}{\lambda-\mu} d \lambda \rightarrow 0
$$

à medida que $x_{0} \rightarrow-\infty$, ou mais informalmente, a reta $R$ é transladada para a esquerda. 
Analogamente, para $R$ fechando $\Gamma^{\prime}, R: x_{0}+$ is, $s \in[-y, y]$, onde $x_{0}<0 e|y|=-\tan (\theta-\delta)\left|x_{0}\right|$, se $\lambda \in \Gamma$, então,

$$
\int_{R} \frac{e^{\mu s}}{\mu-\lambda} d \mu \rightarrow 0
$$

quando $x_{0} \rightarrow-\infty$.

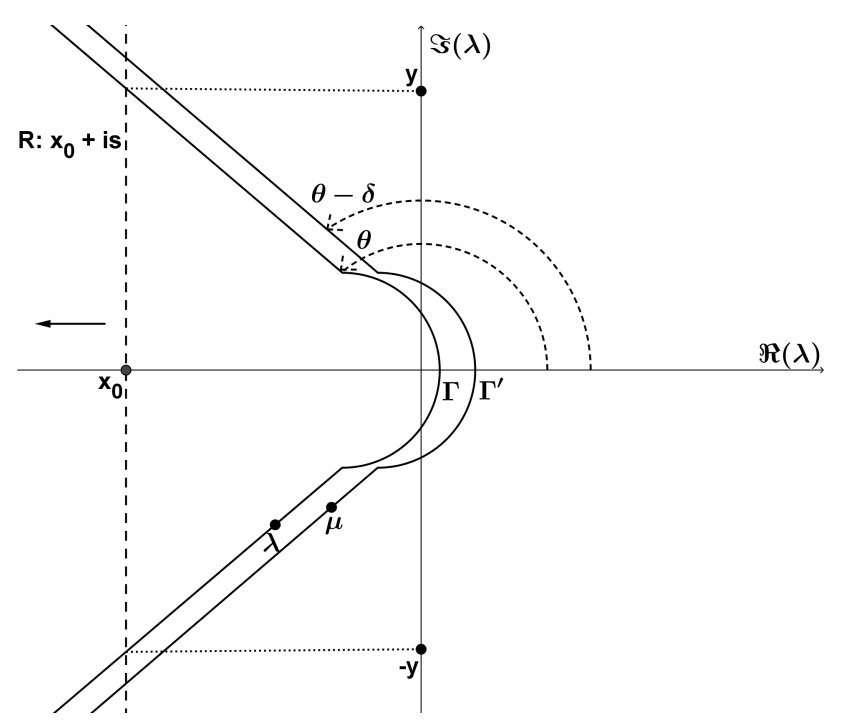

Figura 1.8: Reta $R$ e representação simplificada dos contornos $\Gamma$ e $\Gamma^{\prime}$.

\section{Demonstração:}

Basta demonstrarmos um dos casos, o outro segue de forma análoga. Vejamos,

$$
\begin{aligned}
\left|\int_{R} \frac{e^{\lambda t}}{\lambda-\mu} d \lambda\right| & \leq \int_{R} \frac{e^{\Re(\lambda t)}}{|\lambda-\mu|}|d \lambda| \\
& \leq \int_{R} \frac{e^{|\lambda t| \cos (\arg \lambda)}}{|\lambda-\mu|}|d \lambda| \\
& \leq \int_{R} \frac{e^{|\lambda t| \cos \theta}}{|\lambda-\mu|}|d \lambda| \\
& \leq \int_{R} \frac{e^{|\lambda t| \cos \theta}}{|\lambda|-|\mu|}|d \lambda|
\end{aligned}
$$

que converge à zero quando $R$ se desloca para a esquerda afinal, $|\lambda| \rightarrow \infty$ e $\cos \theta<0$ (usamos que $\cos (\arg \lambda)$ varia entre $\cos \theta$ e $\cos \pi)$.

\section{Demonstração do Teorema 1.1.9:}

Como A é um operador setorial, existem $\phi \in\left(0, \frac{\pi}{2}\right), C \geq 1$ e $a \in \mathbb{R}$ tais que $S_{a, \phi}=\{\lambda \in \mathbb{C} \mid \phi \leq 1$ $\arg (\lambda-a) \mid \leq \pi, \lambda \neq a\} \subset \rho(A)$ e vale que $\left\|(\lambda I-A)^{-1}\right\| \leq \frac{C}{|\lambda-a|}, \forall \lambda \in S_{a, \phi}$ (vide Figura 1.9): 


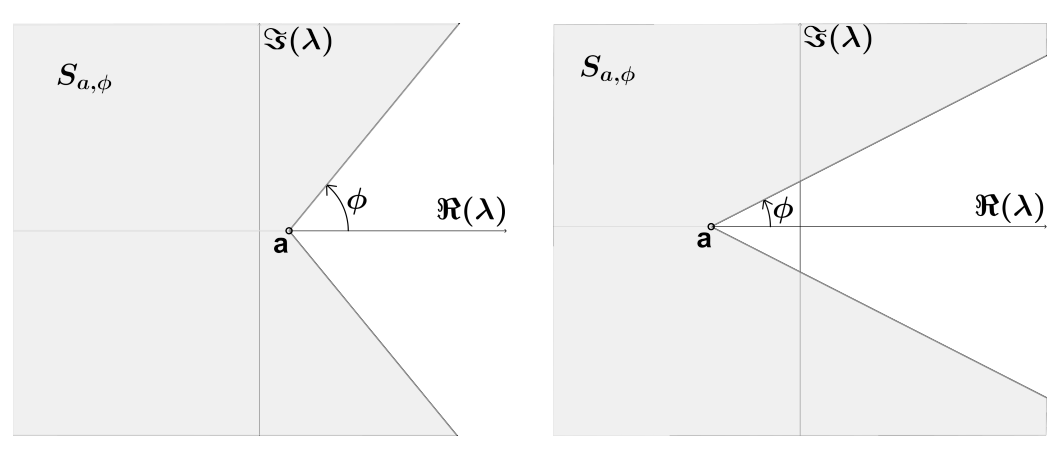

Figura 1.9: Possíveis setores para A.

Sendo as respectivas situações para $-A$ conforme Figura 1.10:
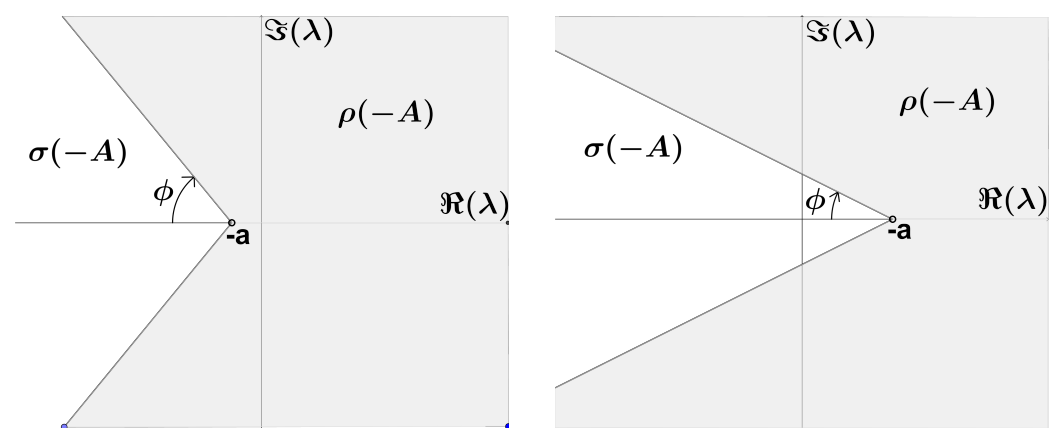

Figura 1.10: Possíveis situações para -A.

Observemos que de acordo com a Definição 1.1.1. o vértice $a$ não necessariamente pertence ao setor $S_{a, \phi}$. Mas, sempre podemos supor isso, transladando o setor um pouco para a esquerda e obtendo um novo setor $S_{a^{\prime}, \phi}$ com $a^{\prime}<a$. Adicionalmente, podemos admitir sem perda de generalidade que $a=0$ e $\left\|(-\lambda I-A)^{-1}\right\|=\left\|(\lambda I+A)^{-1}\right\| \leq \frac{M}{|\lambda|+\delta}$ para $|\pi-\arg (\lambda)| \geq \phi$ e constantes $M>0$ e $\delta>0$. Caso contrário, se $a \neq 0$, basta considerarmos o operador $A-a I$ e, para $\mu=\lambda-a$, $\lambda \in S_{a, \phi}$, temos,

$$
\left\|(\mu I-(A-a I))^{-1}\right\|=\left\|((\lambda-a) I-(A-a I))^{-1}\right\|=\left\|(\lambda I-A)^{-1}\right\| \leq \frac{M}{|\lambda-a|+\delta}=\frac{M}{|\mu|+\delta},
$$

onde $M$ e $\delta$ são tomadas convenientemente.

Mais detalhadamente, se $|\mu|>C$, basta tomar $\delta<C$ e $M=2 C$, pois

$$
\begin{aligned}
\frac{C}{|\mu|} & \leq \frac{M}{|\mu|+\delta} \\
& \Longleftrightarrow M \geq C \frac{|\mu|+\delta}{|\mu|} \\
& \Longleftrightarrow M \geq C\left(1+\frac{\delta}{|\mu|}\right) .
\end{aligned}
$$


E no caso $|\mu| \leq C$, a aplicação $\lambda \in \rho(A) \longmapsto\left\|(\lambda I-A)^{-1}\right\|$ é contínua e, portanto, quando restrita ao compacto $B_{C}(0) \cap S_{a, \phi}-B_{C}(0)$ a bola de raio $C$ e centro na origem -, possui um máximo $N$, ou seja, $\left\|(\lambda I-A)^{-1}\right\| \leq N, \forall \lambda \in B_{C}(0) \cap S_{a, \phi}$. Daí, escolhendo $M$ tal que $\frac{M}{|\mu|+\delta} \geq N$, ou ainda, $M \geq N(C+\delta)$, temos o desejado.

Fazendo $M=\max \{2 C, N(C+\delta)\}$, vale que $\left\|(\lambda I-A)^{-1}\right\| \leq \frac{M}{|\lambda-a|+\delta}=\frac{M}{|\mu|+\delta}$.

Neste ponto, escolha $\theta \in\left(\frac{\pi}{2}, \pi-\phi\right)$ e, estabeleça:

$$
e^{-A t}=\frac{1}{2 \pi i} \int_{\Gamma}(\lambda I+A)^{-1} e^{\lambda t} d \lambda
$$

sendo $\Gamma$ um contorno em $\rho(-A) \operatorname{com} \arg (\lambda) \rightarrow \pm \theta$ quando $|\lambda| \rightarrow \infty$, como ilustra a Figura 1.11 .

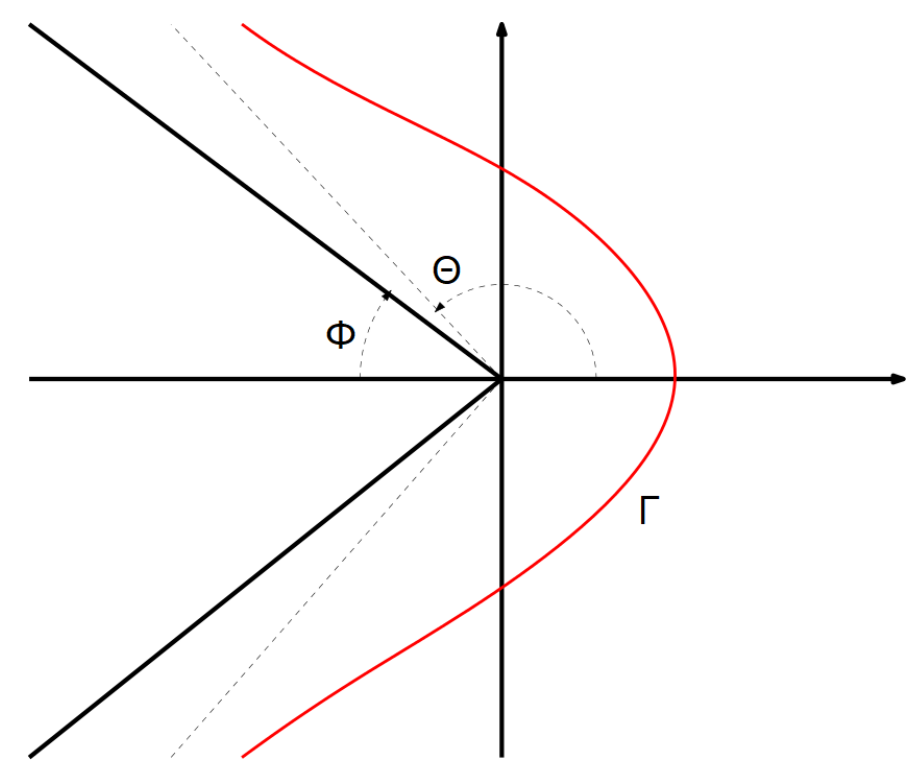

Figura 1.11: Contorno $\Gamma$.

A priori, precisamos verificar que tal definição faz sentido, isto é, que essa integral realmente converge. Vejamos:

Parametrizando a curva $\Gamma$ pelo comprimento de arco, $\gamma:]-\infty,+\infty[\rightarrow \mathbb{C}$,

$$
\begin{aligned}
\left\|\frac{1}{2 \pi i} \int_{\Gamma}(\lambda I+A)^{-1} e^{\lambda t} d \lambda\right\| & =\left\|\frac{1}{2 \pi i} \int_{-\infty}^{+\infty}(\gamma(s)+A)^{-1} e^{\gamma(s) t} \gamma^{\prime}(s) d s\right\| \\
& \leq \frac{1}{2 \pi} \int_{-\infty}^{+\infty}\left\|(\gamma(s)+A)^{-1}\right\| e^{\mathfrak{R}(\gamma(s)) t}\left|\gamma^{\prime}(s) \| d s\right| \\
& \leq D \int_{-\infty}^{+\infty} e^{\mathfrak{R}(\gamma(s)) t} d s \\
& =D \lim _{R \rightarrow \infty}\left(\int_{-R}^{0} e^{\mathfrak{R}(\gamma(s)) t} d s+\int_{0}^{R} e^{\mathfrak{R}(\gamma(s)) t} d s\right) \\
& =2 D \lim _{R \rightarrow \infty} \int_{0}^{R} e^{\mathfrak{R}(\gamma(s)) t} d s,
\end{aligned}
$$


onde $D$ representa uma constante. Notemos que podemos escrever $\gamma(s)=\gamma\left(s_{0}\right)+\int_{s_{0}}^{s} \gamma^{\prime}(t) d t$, sendo $s_{0}$ fixado. Então, $\mathfrak{R}(\gamma(s))=\mathfrak{R}\left(\gamma\left(s_{0}\right)\right)+\int_{s_{0}}^{s} \mathfrak{R}\left(\gamma^{\prime}(t)\right) d t$. Agora, considerando um ângulo $\theta^{\prime}$ tal que $\frac{\pi}{2}<\theta^{\prime}<\theta$, temos que $\mathfrak{R}\left(\gamma^{\prime}(s)\right) \leq-\cos \left(\pi-\theta^{\prime}\right)$ e, consequentemente, $\mathfrak{R}(\gamma(s)) \leq \mathfrak{R}\left(\gamma\left(s_{0}\right)\right)-$ $\cos \left(\pi-\theta^{\prime}\right)\left(s-s_{0}\right)$. Aplicando isso na desigualdade (1.2), vemos claramente a convergência da integral no infinito.

Além disso, a integral independe do caminho $\Gamma$, se deslocarmos $\Gamma$ um pouco para a direita, por exemplo, como na Figura 1.12, ela não se altera.

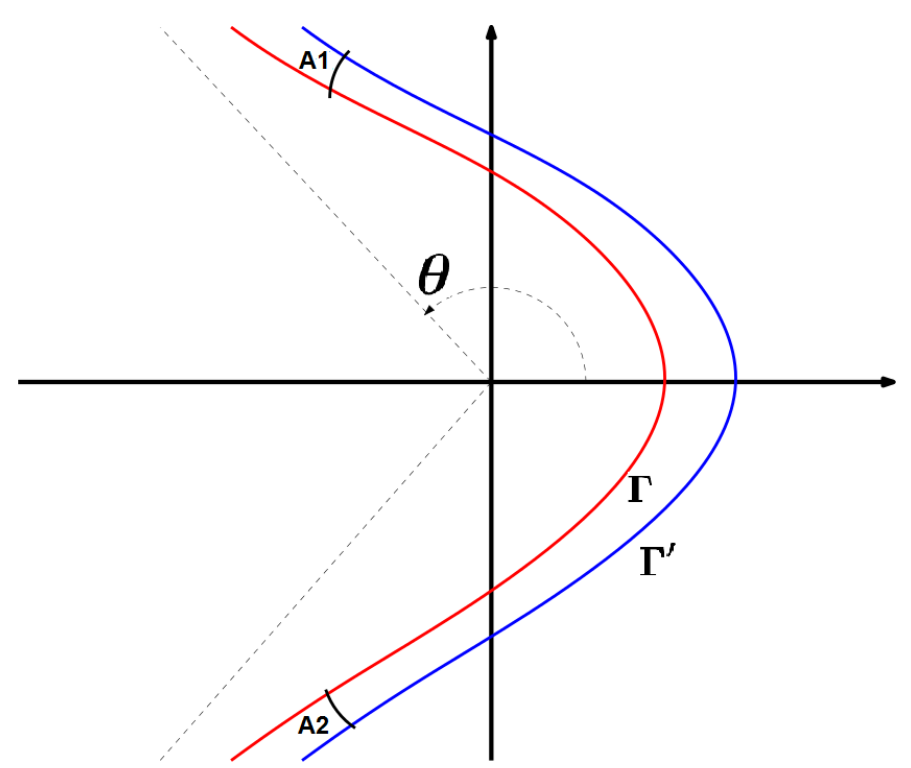

Figura 1.12: Deslocamento de $\Gamma$ para a direita.

De fato, sabe-se que $e^{\lambda t}$ é analítica para todo $\lambda$ e que $(\lambda I+A)^{-1}$ é também uma função analítica em $\rho(-A)$, donde se tomarmos a curva fechada indicada na Figura 1.12 , obtida a partir de $\Gamma, \Gamma^{\prime} \mathrm{e}$ os arcos $A_{1}, A_{2}$, a integral nessa curva é nula. E, fazendo $|\lambda| \rightarrow \infty$, vemos que a integral nesses arcos vai a zero.

Mostraremos a seguir que $e^{-A t} e^{-A s}=e^{-A(t+s)}, \forall t, s>0$. Consideremos para tanto um pequeno deslocamento $\Gamma^{\prime}$ de $\Gamma$ para a direita, conforme Figura 1.12 .

Então, para $t, s>0$,

$$
\begin{aligned}
e^{-A t} e^{-A s} & =\left(\frac{1}{2 \pi i} \int_{\Gamma}(\lambda I+A)^{-1} e^{\lambda t} d \lambda\right)\left(\frac{1}{2 \pi i} \int_{\Gamma^{\prime}}(\mu I+A)^{-1} e^{\mu s} d \mu\right) \\
& =\frac{1}{(2 \pi i)^{2}} \int_{\Gamma} \int_{\Gamma^{\prime}} e^{\lambda t+\mu s}(\mu-\lambda)^{-1}\left((\lambda I+A)^{-1}-(\mu I+A)^{-1}\right) d \mu d \lambda \\
& =\frac{1}{(2 \pi i)^{2}} \int_{\Gamma}(\lambda I+A)^{-1} e^{\lambda t} d \lambda \int_{\Gamma^{\prime}} \frac{e^{\mu s}}{\mu-\lambda} d \mu \\
& +\frac{1}{(2 \pi i)^{2}} \int_{\Gamma} \frac{e^{\lambda t}}{\lambda-\mu} d \lambda \int_{\Gamma^{\prime}}(\mu I+A)^{-1} e^{\mu s} d \mu
\end{aligned}
$$


onde usamos uma das identidades do resolvente. Analisando cada uma das parcelas (1.3) e (1.4) separadamente,

$$
\int_{\Gamma}(\lambda I+A)^{-1} e^{\lambda t} d \lambda \int_{\Gamma^{\prime}} \frac{e^{\mu s}}{\mu-\lambda} d \mu=2 \pi i \int_{\Gamma}(\lambda I+A)^{-1} e^{\lambda(t+s)} d \lambda
$$

Isso porque, se fecharmos $\Gamma^{\prime}$ a partir da reta $R: x_{0}+i s, s \in[-y, y], x_{0}<0, y \in \mathbb{R}$ conveniente, descrita no Lema 1.1.10, vide Figura1.13, pela Fórmula Integral de Cauchy,

$$
\frac{1}{2 \pi i} \int_{\Gamma^{\prime} \cup R} \frac{e^{\mu s}}{\mu-\lambda} d \mu=e^{\lambda s}
$$

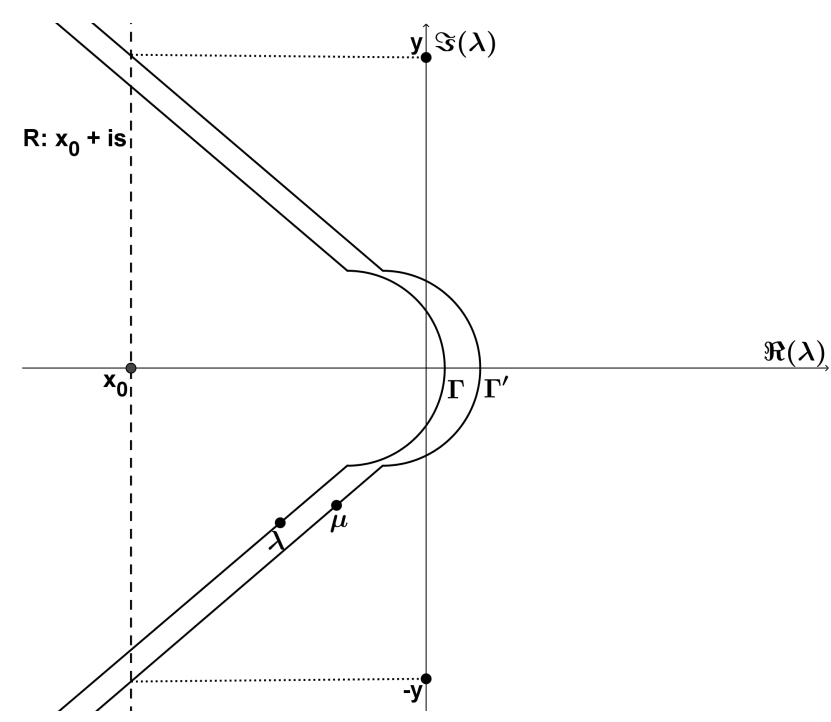

Figura 1.13: Reta R e contornos $\Gamma, \Gamma^{\prime}$.

e, provamos no Lema 1.1 .10 que a integral em $R$ tende a zero à medida que essa reta é deslocada para a esquerda. E,

$$
\int_{\Gamma} \frac{e^{\lambda t}}{\lambda-\mu} d \lambda \int_{\Gamma^{\prime}}(\mu I+A)^{-1} e^{\mu s} d \mu=0,
$$

uma vez que se fecharmos agora $\Gamma$ com o auxílio de $R$, aplicando a Fórmula Integral de Cauchy,

$$
\frac{1}{2 \pi i} \int_{\Gamma \cup R} \frac{e^{\lambda t}}{\lambda-\mu} d \lambda=0
$$

afinal, " $\mu$ está fora de $\Gamma \cup R$ "e, novamente, a integral em $R$ converge a zero quando $R$ é transladada para a esquerda.

Logo, $e^{-A t} e^{-A s}=\frac{1}{2 \pi i} \int_{\Gamma}(\lambda I+A)^{-1} e^{\lambda(t+s)} d \lambda=e^{-A(t+s)}$.

Para mostrar que a aplicação $t \longmapsto e^{-A t} x$ é real analítica em $0<t<\infty$ para cada $x \in X$, verificaremos que aquela integral converge uniformemente em qualquer compacto de $\{t \in \mathbb{C}||$ $\arg (t) \mid<\epsilon\}, 0<\epsilon<\theta-\frac{\pi}{2}$ e então, por um resultado conhecido, o semigrupo é analítico neste conjunto que inclui o eixo real. 
Consideremos a seguinte caracterização equivalente do contorno em $\rho(-A)$ :

$$
\gamma_{\theta, r}=\left\{\begin{array}{l}
\gamma_{\theta, r, 1}(s)=s e^{i\left(\frac{\pi}{2}+\eta\right)}, s \in[r,+\infty) \\
\gamma_{\theta, r, 2}(s)=r e^{i s},|s| \leq \theta \\
\left.\left.\gamma_{\theta, r, 3}(s)=s e^{-i\left(\frac{\pi}{2}+\eta\right)}, s \in\right]-\infty,-r\right] .
\end{array}\right.
$$

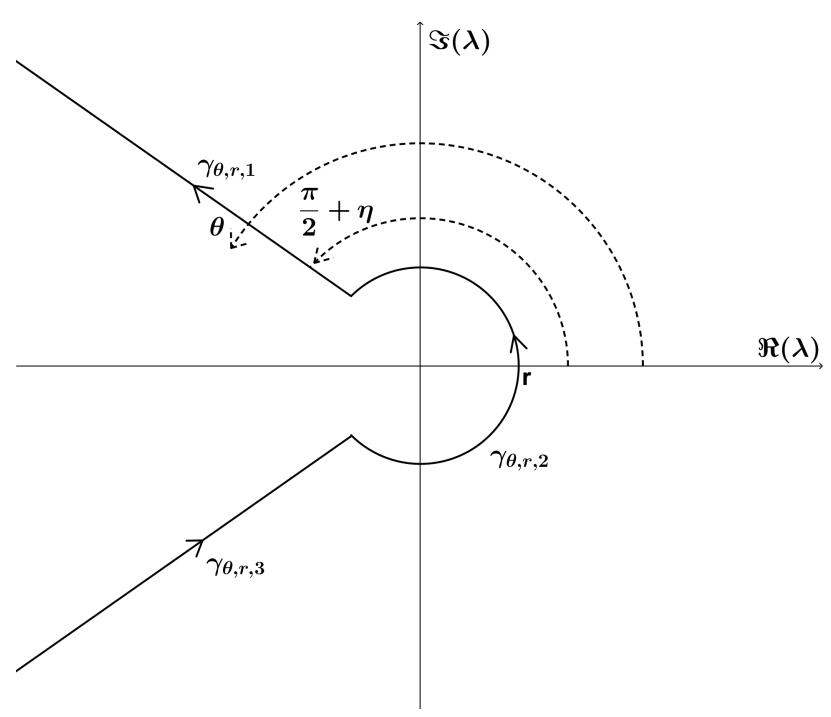

Figura 1.14: Contorno $\gamma_{\theta, r}$.

Sejam $t \in \mathbb{C} \operatorname{com}|\arg (t)|<\epsilon<\frac{\pi}{2} \mathrm{e}, \lambda \in \mathbb{C} \operatorname{com}|\arg (\lambda)|=\frac{\pi}{2}+\eta, \eta \in\left(\frac{|\arg (t)|+\epsilon}{2}, \epsilon\right)$. Então,

$$
|\arg (t)+\arg (\lambda)|>\frac{\pi}{2}+\frac{|\arg (t)|+\epsilon}{2}-|\arg (t)|=\frac{\pi}{2}+\frac{\epsilon-|\arg (t)|}{2}>\frac{\pi}{2}+(\epsilon-\eta),
$$

visto que $\frac{-\epsilon-|\arg (t)|}{2}>-\eta$. E,

$$
|\arg (t)+\arg (\lambda)| \leq \frac{\pi}{2}+\eta+\epsilon \leq \pi-(\epsilon-\eta)+\epsilon \leq \frac{3 \pi}{2}-(\epsilon-\eta) .
$$

Consequentemente,

$$
\left|e^{\lambda t}\right|=e^{\Re(\lambda t)}=e^{|\lambda t| \cos (\arg (t)+\arg (\lambda))} \leq e^{|\lambda t| \cos \left(\frac{\pi}{2}+(\epsilon-\eta)\right)}=e^{|\lambda t|-\sin (\epsilon-\eta)} .
$$

Daí, para justificar a convergência da integral, por exemplo, basta trabalharmos apenas com $\gamma_{\eta, r, 1}$ e $\gamma_{\eta, r, 3}$, permitindo estimar:

$$
\left\|(\lambda I+A)^{-1} e^{\lambda t}\right\| \leq e^{-\sin (e-\eta)|\lambda t|} \frac{M}{|\lambda|+\delta}
$$

que converge a zero exponencialmente quando $|\lambda|$ tende a $\infty$.

Agora, tomando $r \in\left(0, \frac{1}{|t|}\right], t$ tal que $|\arg (t)|<\epsilon^{\prime}<\epsilon, \eta \in\left(\frac{\epsilon^{\prime}+\epsilon}{2}, \epsilon\right)$, temos para $\lambda \in \mathbb{C}$ com $|\arg (\lambda)|=\frac{\pi}{2}+\eta \mathrm{e}|\lambda| \geq r$,

$$
\left\|(\lambda I+A)^{-1} e^{\lambda t}\right\| \leq e^{-|\lambda t| \sin (\epsilon-\eta)} \frac{M}{|\lambda|+\delta} .
$$


$\mathrm{E}, \operatorname{para}|\lambda|=r \mathrm{e},|\arg (\lambda)| \leq \frac{\pi}{2}+\eta$,

$$
\left\|(\lambda I+A)^{-1} e^{\lambda t}\right\| \leq e^{|\lambda t|} \frac{M}{|\lambda|+\delta} \leq e^{r \cdot \frac{1}{r}} \frac{M}{r+\delta}=\frac{e M}{r+\delta} .
$$

Assim,

$$
\begin{aligned}
\left\|e^{-A t}\right\| & =\left\|\frac{1}{2 \pi i} \int_{\gamma_{\eta, r}}(\lambda I+A)^{-1} e^{\lambda t} d \lambda\right\| \\
& \leq \frac{1}{2 \pi}\left(2 \int_{\gamma_{\eta, r, 1}}\left\|(\lambda I+A)^{-1} e^{\lambda t}\right\| d \lambda+\int_{\gamma_{\eta, r, 2}}\left\|(\lambda I+A)^{-1} e^{\lambda t}\right\| d \lambda\right) \\
& =\frac{M}{\pi} \int_{r}^{\infty} \frac{1}{s+\delta} e^{-s|t| \sin (\epsilon-\eta)} d s+\frac{r e M}{r+\delta} \\
& \leq \frac{M}{\pi} \int_{|t| r}^{\infty} \frac{1}{u} e^{-u \sin (\epsilon-\eta)} d u+e M \\
& =\frac{M}{\pi} \int_{1}^{\infty} \frac{1}{u} e^{-u \sin (\epsilon-\eta)} d u+e M, \quad \forall t \in\left\{t \in \mathbb{C}|| \arg (t) \mid<\epsilon^{\prime}\right\} .
\end{aligned}
$$

Suponha $K \subseteq\{t \in \mathbb{C}|| \arg (t) \mid<\epsilon\}$ um conjunto compacto, $\epsilon^{\prime} \in(0, \epsilon)$ tal que $K \subset\{t \in$ $\left.\mathbb{C}|| \arg (t) \mid<\epsilon^{\prime}\right\}$ e $0<r \leq \inf _{t \in K} \frac{1}{|t|}$. A estimativa anterior mostra que a integral definindo $e^{-A t}$ converge uniformemente em $\mathrm{Ke}$, como $t \rightarrow(\lambda I+A)^{-1} e^{\lambda t}$ é analítica, segue que $t \rightarrow e^{-A t} x$ é analítica nesse conjunto, em particular, é real analítica em $0<t<\infty$.

Quanto as desigualdades para as normas dos operadores $e^{-A t}$ e $A e^{-A t}$,

$$
\begin{aligned}
\left\|e^{-A t}\right\| & =\left\|\frac{1}{2 \pi i} \int_{\Gamma}(\lambda I+A)^{-1} e^{\lambda t} d \lambda\right\| \\
& =\left\|\frac{1}{2 \pi i} \int_{\Gamma}\left(\frac{\mu}{t} I+A\right)^{-1} e^{\mu} \frac{d \mu}{t}\right\| \\
& \leq \frac{1}{2 \pi} \int_{\Gamma}\left|e^{\mu}\right|\left\|\left(\frac{\mu}{t} I+A\right)^{-1}\right\|\left|\frac{d \mu}{t}\right| \\
& \leq \frac{M}{2 \pi} \int_{\Gamma}\left|e^{\mu}\right| \frac{|d \mu|}{|\mu|} \\
& =C_{1} .
\end{aligned}
$$

Observemos que apesar da mudança de variável nos cálculos acima, mantivemos a notação para a curva de integração, uma vez que o integrando é analítico e a curva obtida com a mudança $\mu=\lambda t$ 
é admissível. E,

$$
\begin{aligned}
\left\|A e^{-A t}\right\| & =\left\|\frac{1}{2 \pi i} \int_{\Gamma} A(\lambda I+A)^{-1} e^{\lambda t}\right\| \\
& \leq\left\|\frac{1}{2 \pi i} \int_{\Gamma} A\left(\frac{\mu}{t} I+A\right)^{-1} e^{\mu} \frac{d \mu}{t}\right\| \\
& \leq \frac{1}{2 \pi} \int_{\Gamma}\left\|A\left(\frac{\mu}{t} I+A\right)^{-1}\right\|\left|e^{\mu}\right| \frac{|d \mu|}{|t|} \\
& \leq \frac{1+M}{2 \pi} \int_{\Gamma}\left|e^{\mu}\right| \frac{|d \mu|}{|t|} \\
& =\frac{C_{2}}{|t|} \\
& =\frac{C_{2}}{t},
\end{aligned}
$$

onde usamos que,

$$
\begin{aligned}
\left\|A(\lambda I+A)^{-1}\right\| & =\left\|(\lambda I+A)(\lambda I+A)^{-1}-\lambda(\lambda I+A)^{-1}\right\| \\
& =\left\|I-\lambda(\lambda I+A)^{-1}\right\| \\
& \leq\|I\|+|\lambda|\left\|(\lambda I+A)^{-1}\right\| \\
& \leq 1+|\lambda| \frac{M}{|\lambda|+\delta} \\
& <1+M .
\end{aligned}
$$

É muito importante para o nosso trabalho notarmos que as constantes $C_{1}$ e $C_{2}$ dependem exclusivamente de $M$ e do setor para $\mathrm{A}$, os quais variam "continuamente"com o operador.

Caso $\mathfrak{R}(\sigma(A))>a>0,(\mathfrak{R}(\sigma(-A))<-a)$, por exemplo, conforme discutimos no início, consideramos a translação $A-a \mathrm{e}$, dessa forma, $\lambda=\mu-a$,

$$
\frac{1}{2 \pi i} \int_{\Gamma}(\lambda I+A)^{-1} e^{\lambda t} d \lambda=\frac{1}{2 \pi i} \int_{\Gamma^{\prime}}(\mu I-(-A+a))^{-1} e^{-a t} e^{\mu t} d \mu=e^{-a t} e^{(-A+a) t}
$$

e obtemos as desigualdades $\left\|e^{-A t}\right\| \leq e^{-a t} C_{1}$ e $\left\|A e^{-A t}\right\| \leq e^{-a t} \frac{C_{2}}{t}$, sendo $C_{1}$ e $C_{2}$ as constantes especificadas anteriormente.

Esses cálculos nos mostram que ambos os operadores $e^{-A t}$ e $A e^{-A t}$ são limitados para $t>0$, estando definidos em todo espaço $X$.

No que segue, provaremos que $e^{-A t} x \rightarrow x$ quando $t \rightarrow 0^{+}$para cada $x \in X$. Como $e^{-A t}$ é um operador contínuo (uniformemente em t) e $D(A)$ é denso em $X$, é suficiente constatar que isto vale para cada $x \in D(A)$,

$$
\begin{aligned}
e^{-A t} x-x & =\frac{1}{2 \pi i} \int_{\Gamma}(\lambda I+A)^{-1} e^{\lambda t} x d \lambda-x \\
& =\frac{1}{2 \pi i} \int_{\Gamma}\left((\lambda I+A)^{-1}-\lambda^{-1}\right) e^{\lambda t} x d \lambda \\
& =-\frac{1}{2 \pi i} \int_{\Gamma}\left(\lambda^{-1} A(\lambda I+A)^{-1}\right) e^{\lambda t} x d \lambda,
\end{aligned}
$$


onde aplicamos a Fórmula Integral de Cauchy para $\frac{1}{2 \pi i} \int_{\Gamma \cup R} \frac{e^{\lambda t}}{\lambda-0} d \lambda=e^{0 t}$ conforme argumento anterior e, também, que $-\lambda^{-1} A(\lambda I+A)^{-1}=-\lambda^{-1}\left((\lambda I+A)(\lambda I+A)^{-1}-\lambda(\lambda I+A)^{-1}\right)=-\lambda^{-1}+(\lambda I+A)^{-1}$. Tomando então a norma, obtemos:

$$
\begin{aligned}
\left\|e^{-A t} x-x\right\| & \leq \frac{1}{2 \pi} \int_{\Gamma}\left\|\lambda^{-1}(\lambda I+A)^{-1} A x e^{\lambda t} d \lambda\right\| \\
& =\frac{1}{2 \pi} \int_{\Gamma}\left\|\frac{t}{\mu}\left(\frac{\mu}{t} I+A\right)^{-1} A x e^{\mu} \frac{d \mu}{t}\right\| \\
& \leq \frac{M}{2 \pi}\|A x\| \int_{\Gamma}\left|e^{\mu}\right| \frac{|t|}{|\mu|^{2}}|d \mu| \\
& =\text { Constante }\|A x\| \cdot t \rightarrow 0, \text { quando } t \rightarrow 0^{+} .
\end{aligned}
$$

Logo, $\left\{e^{-A t}\right\}_{t \geq 0}$ é um semigrupo fortemente contínuo que pode ser estendido a um semigrupo analítico em $\{t \in \mathbb{C}|| \arg (t) \mid<\epsilon\}, 0<\epsilon<\theta-\frac{\pi}{2}$.

Finalmente, com relação às últimas considerações, sejam $x \in D(A), t>0$. Devido à analiticidade e à convergência uniforme, temos:

$$
\frac{d}{d t} e^{-A t} x+A e^{-A t} x=\frac{1}{2 \pi i} \int_{\Gamma} e^{\lambda t}\left(\lambda(\lambda I+A)^{-1}+A(\lambda I+A)^{-1}\right) d \lambda x=\frac{1}{2 \pi i} \int_{\Gamma} e^{\lambda t} d \lambda x
$$

e,

$$
\int_{\Gamma} e^{\lambda t} d \lambda=0
$$

Isso porque se fecharmos $\Gamma$ com o auxílio da reta $\mathrm{R}$ mencionada, $\operatorname{como} e^{\lambda t}$ não tem singularidades, pelo Teorema de Cauchy,

$$
\int_{\Gamma \cup R} e^{\lambda t} d \lambda=0
$$

e, basta constatarmos que a integral em $\mathrm{R}$ tende a zero a medida que essa reta é deslocada para a esquerda. Com efeito,

$$
\left|\int_{R} e^{\lambda t} d \lambda\right| \leq \int_{R}\left|e^{\lambda t}\right||d \lambda|=\int_{R} e^{|\lambda t| \cos (\arg \lambda)}|d \lambda| \leq \int_{R} e^{|\lambda t| \cos (\theta-\delta)}|d \lambda|
$$

onde $\delta$ é uma constante positiva pequena. Observamos que $\cos (\theta-\delta)$ é negativo de modo que conforme $|\lambda|$ aumenta (a reta $\mathrm{R}$ é transladada para a esquerda), o integrando converge a zero exponencialmente.

Portanto, $\frac{d}{d t} e^{-A t} x=-A e^{-A t} x, \forall x \in D(A)$.

Por conseguinte, para $x \in D(A)$,

$$
\frac{1}{t}\left(e^{-A t} x-x\right)=-\frac{1}{t} \int_{0}^{t} e^{-A s} A x d s=-\frac{1}{t} \int_{0}^{t}\left(e^{-A s}-I\right) A x+A x d s \rightarrow-A x, \text { quando } t \rightarrow 0^{+} .
$$

Isso mostra que $-A$ está contido no gerador $\mathrm{G}$ do semigrupo pela Definição 1.1.7. Resta então a inclusão contrária; $G \subseteq-A$. 
Para tanto, defina para cada $\lambda \geq 0$ e $x \in X$,

$$
R(\lambda) x=\int_{0}^{\infty} e^{-\lambda t} e^{-A t} x d t
$$

Vejamos que essa integral de fato existe, supondo $\mathfrak{R}(\sigma(A))>\delta>0$,

$$
\left\|\int_{0}^{\infty} e^{-\lambda t} e^{-A t} x d t\right\| \leq \int_{0}^{\infty}\left|e^{-\lambda t}\right|\left\|e^{-A t} x\right\||d t| \leq C_{1}\|x\| \int_{0}^{\infty} e^{-\lambda t} e^{-\delta t} d t
$$

e,

$$
C_{1}\|x\| \int_{0}^{\infty} e^{(-\delta-\lambda) t} d t=\left.C_{1}\|x\| \frac{-1}{\delta+\lambda} e^{(-\delta-\lambda) t}\right|_{0} ^{\infty}=C_{1}\|x\| \frac{1}{\delta+\lambda} .
$$

Para todo $x, e^{-A t} x \in D(A) \operatorname{com} t>0$, uma vez que $A e^{-A t}$ é um operador limitado se $t>0$ como vimos. Assim, se $R_{\delta}(\lambda) x=\int_{\delta}^{\infty} e^{-\lambda t} e^{-A t} x d t, \delta>0$,

$$
A \int_{\delta}^{\infty} e^{-\lambda t} e^{-A t} x d t=\int_{\delta}^{\infty} e^{-\lambda t} A e^{-A t} x d t=-\left.e^{-\lambda t} e^{-A t} x\right|_{\delta} ^{\infty}-\lambda \int_{\delta}^{\infty} e^{-\lambda t} e^{-A t} x d t
$$

Ou seja,

$$
A\left(R_{\delta}(\lambda) x\right)=e^{-\lambda \delta} e^{-A \delta} x-\lambda \int_{\delta}^{\infty} e^{-\lambda t} e^{-A t} x d t
$$

e, quando $\delta \rightarrow 0^{+}, R_{\delta}(\lambda) x$ converge a $R(\lambda) x$ enquanto $A\left(R_{\delta}(\lambda) x\right)$ tende a $x-\lambda R(\lambda) x$, o que significa, sendo A um operador fechado, que $R(\lambda) x \in D(A) \subset D(G), \forall \lambda \geq 0, x \in X$ e $A(R(\lambda) x)=x-\lambda R(\lambda) x$.

E, se $x \in D(G), e^{-A t} x \in D(A) \subset D(G), \forall t \geq 0$, valendo, por um lado, que

$$
G\left(e^{-A t} x\right)=-A e^{-A t} x=\frac{d}{d t} e^{-A t} x .
$$

E, por outro,

$$
G\left(e^{-A t} x\right)=\lim _{\tau \rightarrow 0} \frac{e^{-A \tau}\left(e^{-A t} x\right)-e^{-A t} x}{\tau}=\lim _{\tau \rightarrow 0} \frac{e^{-A t}\left(e^{-A \tau} x-x\right)}{\tau}=e^{-A t} \lim _{\tau \rightarrow 0} \frac{e^{-A \tau} x-x}{\tau}=e^{-A t} G x .
$$

Aplicando isso, se $x \in D(G)$,

$$
\begin{aligned}
R(\lambda)(\lambda-G) x & =\int_{0}^{\infty} e^{-\lambda t} e^{-A t} \lambda x d t-\int_{0}^{\infty} e^{-\lambda t} e^{-A t} G x d t \\
& =-\left.e^{-\lambda t} e^{-A t} x\right|_{0} ^{\infty}-\int_{0}^{\infty} e^{-\lambda t} A e^{-A t} x d t-\int_{0}^{\infty} e^{-\lambda t} e^{-A t} G x d t \\
& =x
\end{aligned}
$$

pois $A e^{-A t} x=-e^{-A t} G(x)$, combinando as igualdades (1.6) e (1.7). Logo, $R(\lambda)(\lambda-G) x=x, \forall x \in D(G)$ e, segue que, $D(G) \subseteq R(R(\lambda)) \subseteq D(A)$ donde concluimos o desejado; - A é o gerador infinitesimal de $\left\{e^{-A t}\right\}_{t \geq 0}$. 


\subsection{Potências fracionárias de operadores}

Nesta seção, primeiramente, definimos as potências fracionárias, $A^{\alpha}, \alpha \in \mathbb{R}$, de um operador setorial $A$ e estudamos algumas de suas propriedades para então introduzirmos os espaços fracionários, $X^{\alpha}=D\left(A_{1}^{\alpha}\right)$, onde $\alpha \geq 0, A_{1}=A+a$, com $a$ tal que $\mathfrak{R}\left(\sigma\left(A_{1}\right)\right)>0$, nos quais iremos trabalhar.

Definição 1.2.1 Seja A um operador setorial com $\mathfrak{R}(\sigma(A))>0$, então para qualquer $\alpha>0$, definimos:

$$
A^{-\alpha}=\frac{1}{\Gamma(\alpha)} \int_{0}^{+\infty} t^{\alpha-1} e^{-A t} d t
$$

onde $\Gamma$ denota a função Gama dada por $\Gamma(\alpha)=\int_{0}^{\infty} x^{\alpha-1} e^{-x} d x$.

Vale observar que de fato essa definição faz sentido, isto é, a expressão que aparece na Definição 1.2.1 é finita.

Como $\mathfrak{R}(\sigma(A))>0$, podemos dizer que $\mathfrak{R}(\sigma(A))>\varepsilon$ para algum $\varepsilon>0$ e pelo Teorema 1.1.9.

$$
\left\|t^{\alpha-1} e^{-A t}\right\| \leq t^{\alpha-1} C_{1} e^{-\varepsilon t}, \forall t>0 .
$$

Aplicando isso,

$$
\begin{aligned}
\left\|\int_{0}^{+\infty} t^{\alpha-1} e^{-A t} d t\right\| & \leq \int_{0}^{+\infty}\left\|t^{\alpha-1} e^{-A t}\right\| d t \\
& \leq C_{1} \int_{0}^{+\infty} t^{\alpha-1} e^{-\varepsilon t} d t \\
& =C_{1} \int_{0}^{+\infty}\left(\frac{s}{\varepsilon}\right)^{\alpha-1} e^{-s} \frac{d s}{\varepsilon} \\
& =C_{1} \varepsilon^{-\alpha} \int_{0}^{+\infty} s^{\alpha-1} e^{-s} d s .
\end{aligned}
$$

A integral em (1.8) é finita, pois

$$
\int_{0}^{1} s^{\alpha-1} e^{-s} d s \leq \int_{0}^{1} s^{\alpha-1} d s=\frac{1}{\alpha}
$$

e,

$$
\begin{aligned}
\int_{1}^{r} s^{\alpha-1} e^{-s} d s & \leq \int_{1}^{r} n ! s^{-n} s^{\alpha-1} d s \\
& =n ! \int_{1}^{r} s^{\alpha-n-1} d s \\
& =\frac{n !}{\alpha-n}\left(r^{\alpha-n}-1\right),
\end{aligned}
$$

onde $n$ é um inteiro positivo com $n>\alpha$ e pela expansão em série de Taylor de $e^{s}$, temos $e^{s}>\frac{s^{n}}{n !}$. Fazendo $r \rightarrow \infty$, vemos que existe a integral,

$$
\int_{1}^{+\infty} s^{\alpha-1} e^{-s} d s
$$


Logo,

$$
\int_{0}^{+\infty} s^{\alpha-1} e^{-s} d s=\int_{0}^{1} s^{\alpha-1} e^{-s} d s+\int_{1}^{+\infty} s^{\alpha-1} e^{-s} d s
$$

Exemplo 1.2.2 Se $X=\mathbb{R}$ e A é um escalar positivo, $A=a$, então $A^{-\alpha}$ como definido coincide com a (- $\left.\alpha\right)$ potência usual de A.

Com efeito,

$$
\begin{aligned}
A^{-\alpha} & :=\frac{1}{\Gamma(\alpha)} \int_{0}^{+\infty} t^{\alpha-1} e^{-a t} d t \\
& =\frac{1}{\Gamma(\alpha)} \int_{0}^{+\infty}\left(\frac{s}{a}\right)^{\alpha-1} e^{-s} \frac{d s}{a} \\
& =\frac{1}{\Gamma(\alpha)} a^{-\alpha} \int_{0}^{+\infty} s^{\alpha-1} e^{-s} d s \\
& =\frac{1}{\Gamma(\alpha)} a^{-\alpha} \Gamma(\alpha) \\
& =a^{-\alpha} .
\end{aligned}
$$

Exemplo 1.2.3 $A^{-1}$ (isto é, o caso $\alpha=1$ ) é a inversa de A.

Vejamos:

$$
\begin{aligned}
A^{-1} & :=\frac{1}{\Gamma(1)} \int_{0}^{+\infty} t^{1-1} e^{-A t} d t \\
& =\int_{0}^{+\infty} e^{-A t} d t
\end{aligned}
$$

e, usando os resultados do Teorema 1.1 .9 e o fato de $0 \in \rho(A)$, temos:

$$
\begin{aligned}
\int_{0}^{+\infty} e^{-A t} x d t & =\int_{0}^{+\infty} A^{-1} A e^{-A t} x d t \\
& =A^{-1} \int_{0}^{+\infty} \frac{d}{d t}\left(-e^{-A t} x\right) d t \\
& =A^{-1} x, \forall x \in X,
\end{aligned}
$$

donde se verifica que o caso $\alpha=1$ realmente descreve a inversa de $A$.

Exemplo 1.2.4 Se $A=I+B$, onde $\|B\|<1$, então $A^{-\alpha}$ como definido acima concorda com a representação usual em série de potências: $(I+B)^{-\alpha}=\sum_{n=0}^{\infty}\left(\begin{array}{c}-\alpha \\ n\end{array}\right) B^{n}$, sendo $\left(\begin{array}{c}-\alpha \\ n\end{array}\right)=(-1)^{n} \frac{\Gamma(\alpha+n)}{n ! \Gamma(\alpha)}$. 
Conforme vimos no Exemplo 1.1.2, A é setorial por ser um operador limitado. E, aplicando a Definição 1.2.1 e, fazendo alguns cálculos, encontramos:

$$
\begin{aligned}
(I+B)^{-\alpha} & :=\frac{1}{\Gamma(\alpha)} \int_{0}^{\infty} t^{\alpha-1} e^{-(I+B) t} d t \\
& =\frac{1}{\Gamma(\alpha)} \int_{0}^{\infty} t^{\alpha-1} e^{-t}\left(\sum_{n=0}^{\infty} \frac{(-1)^{n} B^{n} t^{n}}{n !}\right) d t \\
& =\frac{1}{\Gamma(\alpha)} \sum_{n=0}^{\infty} \frac{(-1)^{n} B^{n}}{n !} \int_{0}^{\infty} t^{n+\alpha-1} e^{-t} d t \\
& =\sum_{n=0}^{\infty} \frac{(-1)^{n} \Gamma(\alpha+n) B^{n}}{n ! \Gamma(\alpha)} \\
& =\sum_{n=0}^{\infty}\left(\begin{array}{c}
-\alpha \\
n
\end{array}\right) B^{n} .
\end{aligned}
$$

Teorema 1.2.5 Se A é um operador setorial em $X \operatorname{com} \mathfrak{R}(\sigma(A))>0$, então para qualquer $\alpha>0, A^{-\alpha}$ é um operador linear limitado em $X$, injetor, que satisfaz $A^{-\alpha} A^{-\beta}=A^{-(\alpha+\beta)}$ sempre que $\alpha>0, \beta>0$. Além disso, se $0<\alpha<1$,

$$
A^{-\alpha}=\frac{\sin (\pi \alpha)}{\pi} \int_{0}^{+\infty} \lambda^{-\alpha}(\lambda I+A)^{-1} d \lambda
$$

\section{Demonstração:}

Como $\mathfrak{R}(\sigma(A))>0$, podemos dizer que $\mathfrak{R}(\sigma(A))>\delta$ para algum $\delta>0$ e do Teorema 1.1.9. temos $\left\|e^{-A t}\right\| \leq C_{1} e^{-\delta t}, t>0$. Segue que,

$$
\begin{aligned}
\left\|A^{-\alpha} x\right\| & =\left\|\frac{1}{\Gamma(\alpha)} \int_{0}^{\infty} t^{\alpha-1} e^{-A t} x d t\right\| \\
& \leq \frac{1}{\Gamma(\alpha)} \int_{0}^{\infty} t^{\alpha-1}\left\|e^{-A t}\right\|\|x\| d t \\
& \leq \frac{C_{1}}{\Gamma(\alpha)} \int_{0}^{\infty} t^{\alpha-1} e^{-\delta t} d t\|x\| \\
& =C_{1} \delta^{-\alpha}\|x\| .
\end{aligned}
$$

E, constatamos assim que $A^{-\alpha}$ é um operador limitado, lembrando que $C_{1}$ depende do setor e da constante $M$ na desigualdade do resolvente associados ao operador $A$. 
Agora, se $\alpha>0, \beta>0$,

$$
\begin{aligned}
A^{-\alpha} A^{-\beta} & :=\left(\frac{1}{\Gamma(\alpha)} \int_{0}^{\infty} t^{\alpha-1} e^{-A t} d t\right)\left(\frac{1}{\Gamma(\beta)} \int_{0}^{\infty} s^{\beta-1} e^{-A s} d s\right) \\
& =\frac{1}{\Gamma(\alpha) \Gamma(\beta)} \int_{0}^{\infty} \int_{0}^{\infty} t^{\alpha-1} s^{\beta-1} e^{-A(t+s)} d s d t \\
& =\frac{1}{\Gamma(\alpha) \Gamma(\beta)} \int_{0}^{\infty} d u \int_{0}^{u} t^{\alpha-1}(u-t)^{\beta-1} e^{-A u} d t \\
& =\frac{1}{\Gamma(\alpha) \Gamma(\beta)} \int_{0}^{\infty} u^{\alpha+\beta-1} e^{-A u} d u \int_{0}^{1} z^{\alpha-1}(1-z)^{\beta-1} d z \\
& :=A^{-(\alpha+\beta)},
\end{aligned}
$$

onde usamos que

$$
\int_{0}^{1} z^{\alpha-1}(1-z)^{\beta-1} d z=\frac{\Gamma(\alpha) \Gamma(\beta)}{\Gamma(\alpha+\beta)}
$$

Além disso, se $A^{-\alpha} x=0$ para algum $\alpha>0$, então tomando $n \in \mathbb{N}, n>\alpha, A^{-n} x=A^{-(n-\alpha)} A^{-\alpha} x=$ 0 . Ocorre que $\mathfrak{R}(\sigma(A))>0$ significa que $0 \in \rho(A)$, e, portanto, A é inversível ; existe $A^{-1}$ e é injetor de modo que $A^{-n}$, a n-ésima potência de $A^{-1}$, também o é. Logo, $x=0$, o que nos mostra que $A^{-\alpha}$ é injetor qualquer $\alpha>0$.

Por fim, resta ver que, para $0<\alpha<1$,

$$
A^{-\alpha}=\frac{\sin (\pi \alpha)}{\pi} \int_{0}^{+\infty} \lambda^{-\alpha}(\lambda I+A)^{-1} d \lambda
$$

Para $\lambda \geq 0, A+\lambda I$ é setorial com $\mathfrak{R}(\sigma(A+\lambda I))>0$ e, aplicando a Definição 1.2.1.

$$
(A+\lambda I)^{-1}=\int_{0}^{\infty} e^{-A t} e^{-\lambda t} d t
$$

donde,

$$
\begin{aligned}
\int_{0}^{\infty} \lambda^{-\alpha}(\lambda I+A)^{-1} d \lambda & =\int_{0}^{\infty} \lambda^{-\alpha}\left(\int_{0}^{\infty} e^{-A t} e^{-\lambda t} d t\right) d \lambda \\
& =\int_{0}^{\infty} e^{-A t}\left(\int_{0}^{\infty} \lambda^{-\alpha} e^{-\lambda t} d \lambda\right) d t \\
& =\int_{0}^{\infty} e^{-A t} t^{\alpha-1} \Gamma(1-\alpha) d t \\
& =A^{-\alpha} \Gamma(\alpha) \Gamma(1-\alpha) .
\end{aligned}
$$

E, usando que para $0<\alpha<1, \Gamma(\alpha) \Gamma(1-\alpha)=\frac{\pi}{\sin (\pi \alpha)}$, resulta o desejado.

Observação 1.2.6 Vale observar que já sabíamos pela demonstração do Teorema 1.1.9 que a definição da potência fracionária $\alpha=-1$ de $\lambda I+A$ coincide com a sua inversa como operador.

Esse resultado nos permite definir: 
Definição 1.2.7 Seja A como no Teorema 1.2.5 Definimos $A^{\alpha}$, a inversa de $A^{-\alpha}$, para $\alpha>0$, com $D\left(A^{\alpha}\right)=R\left(A^{-\alpha}\right)$ e $A^{0}=I$, onde I é a identidade em X.

Exemplo 1.2.8 $A=A^{1}$ é a inversa de $A^{-1}$.

Teorema 1.2.9 Suponha A um operador setorial $\operatorname{com} \mathfrak{R}(\sigma(A))>\delta>0$. Então, para $\alpha \geq 0$, existe uma constante $0<C_{\alpha}<\infty$ tal que

$$
\left\|A^{\alpha} e^{-A t}\right\| \leq C_{\alpha} t^{-\alpha} e^{-\delta t}, t>0,
$$

onde, se $C_{2}$ é a constante do Teorema 1.1.9.

$$
C_{\alpha}=\left\{\begin{array}{l}
C_{2} \Gamma(\alpha), \text { se } 0<\alpha<1 ; \\
\left(C_{2} m\right)^{m}, \text { se } \alpha=m=1,2,3 \ldots ; \\
C_{m} C_{\beta} 2^{m+\beta}, \text { se } \alpha=m+\beta>1, m \in \mathbb{N}^{*}, 0<\beta<1 ;
\end{array}\right.
$$

e, se $0<\alpha \leq 1$ e $x \in D\left(A^{\alpha}\right)$,

$$
\left\|\left(e^{-A t}-I\right) x\right\| \leq \frac{1}{\alpha} C_{1-\alpha} t^{\alpha}\left\|A^{\alpha} x\right\| .
$$

Além disso, $C_{\alpha}$ é limitada para a em qualquer intervalo compacto de $(0,+\infty)$ e $C_{\alpha}$ depende do setor e da constante $M$ na desigualdade do resolvente para A. (Veremos que $C_{\alpha}$ é também limitada quando $\alpha \rightarrow 0^{+}$).

A demonstração desse teorema exige o conhecimento de algumas propriedades sobre as potências fracionárias, as quais enunciaremos e verificaremos a seguir:

Lema 1.2.10 Seja A um operador setorial. Então,

(i) $D\left(A^{\alpha}\right) \subseteq D\left(A^{\beta}\right)$ para $\alpha, \beta \in \mathbb{R} \operatorname{com} \alpha \geq \beta$.

(ii) $A^{\alpha}$ é fechado e densamente definido para $\alpha>0$.

(iii) $A^{\alpha} e^{-A t}=e^{-A t} A^{\alpha}$ em $D\left(A^{\alpha}\right)$ para $t>0$ e $\alpha \in \mathbb{R}$.

(iv) $A^{\alpha} A^{\beta}=A^{\beta} A^{\alpha}=A^{\alpha+\beta}$ em $D\left(A^{\gamma}\right)$, onde $\alpha, \beta \in \mathbb{R}$ e $\gamma=\operatorname{máx}\{\alpha, \beta, \alpha+\beta\}$.

\section{Demonstração:}

(i) De fato, notemos que mostrar que $D\left(A^{\alpha}\right) \subseteq D\left(A^{\beta}\right)$, para $\alpha, \beta>0$ com $\alpha \geq \beta$, é equivalente a verificar, nessas condições, que $R\left(A^{-\alpha}\right) \subseteq R\left(A^{-\beta}\right)$. Como $\alpha \geq \beta$ e são ambos positivos, aplicando o último Teorema 1.2.5. $A^{-\alpha}=A^{-\beta} A^{-(\alpha-\beta)}$, ou seja, $R\left(A^{-\alpha}\right) \subseteq R\left(A^{-\beta}\right)$. Para $\alpha, \beta \leq 0$, o domínio de 
ambos os operadores é todo X. E se $\alpha>0, \beta<0$, então $D\left(A^{\alpha}\right) \subset X=D\left(A^{\beta}\right)$.

(ii) Sabemos que $A^{\alpha}=\left(A^{-\alpha}\right)^{-1}$, sendo $A^{-\alpha}$ um operador limitado, $D\left(A^{-\alpha}\right)=X$ e, portanto, fechado.

Seja $x_{n} \in D\left(A^{\alpha}\right)=R\left(A^{-\alpha}\right) \operatorname{com} x_{n} \rightarrow x$ e $y_{n}=A^{\alpha} x_{n} \rightarrow y$. Então, $\left(x_{n}, y_{n}\right) \in \operatorname{Graf}\left(A^{\alpha}\right)$ se, e somente se, $\left(y_{n}, x_{n}\right) \in \operatorname{Graf}\left(A^{-\alpha}\right)$. Dado que o gráfico de $A^{-\alpha}$ é fechado, $(y, x) \in \operatorname{Graf}\left(A^{-\alpha}\right)$ e, consequentemente, $(x, y) \in \operatorname{Graf}\left(A^{\alpha}\right)$.

Agora, para a segunda parte, afirmo que é suficiente constatarmos que se $A$ é um operador setorial e $m \in \mathbb{N}$, então $D\left(A^{m}\right)$ é denso em X. Isso porque, se $\alpha \geq 1$, existe $m \in \mathbb{N}$ tal que $m \geq \alpha$ e, $D\left(A^{m}\right) \subseteq D\left(A^{\alpha}\right)$ usando (i). E, caso, $0<\alpha<1, D(A) \subseteq D\left(A^{\alpha}\right)$ por (i) novamente.

Seja $x \in D(A)$. Uma vez que $\overline{D(A)}=X$, dado qualquer $\epsilon>0$, podemos encontrar $y \in D(A)$ tal que, $\|y-A x\| \leq \frac{\epsilon}{\left\|A^{-1}\right\|}$. Assim, se $z=A y$, então $\left\|A^{-2} z-x\right\|=\left\|A^{-1} y-A^{-1}(A x)\right\| \leq\left\|A^{-1}\right\| \|$ $y-A x \| \leq \epsilon$. Logo, $\overline{D\left(A^{2}\right)} \supseteq D(A)$ e, portanto, $\overline{D\left(A^{2}\right)} \supset \overline{D(A)}=X ; D\left(A^{2}\right)$ é denso em X. Por indução, mostra-se que $D\left(A^{m}\right)$ é denso em $X, \forall m \geq 1$.

(iii) Para $\alpha>0$, como

$$
\begin{aligned}
A^{-\alpha} e^{-A s} x & =\frac{1}{\Gamma(\alpha)} \int_{0}^{\infty} t^{\alpha-1} e^{-A t} e^{-A s} x d t \\
& =\frac{1}{\Gamma(\alpha)} \int_{0}^{\infty} t^{\alpha-1} e^{-A s} e^{-A t} x d t \\
& =e^{-A s} \frac{1}{\Gamma(\alpha)} \int_{0}^{\infty} t^{\alpha-1} e^{-A t} x d t \\
& =e^{-A s} A^{-\alpha} x, \forall x \in X, s>0
\end{aligned}
$$

afinal, $e^{-A s}$ é contínuo pelo Teorema 1.1.9. $A^{-\alpha}$ e $e^{-A t}$ comutam, $\forall t>0$. E, é também verdade que dados dois operadores $C$ e $D$ que comutam e $C$ é inversível, então $D$ comuta $\operatorname{com} C^{-1}$. Concluimos assim que $A^{\alpha} e^{-A t}=e^{-A t} A^{\alpha}$ em $D\left(A^{\alpha}\right)$ para $\alpha>0$ e $\forall t>0$. Observemos que da primeira linha desse raciocínio segue diretamente o caso em que o expoente é negativo.

(iv) Analisemos os possíveis casos abaixo:

Caso 1: $\alpha, \beta>0$.

Se $x \in D\left(A^{\alpha} A^{\beta}\right)$, então, $x \in D\left(A^{\beta}\right)$ e $A^{\beta} x \in D\left(A^{\alpha}\right)$. E, do Teorema 1.2.5, $A^{\alpha} A^{\beta} x=y$ implica que $x=A^{-\beta} A^{-\alpha} y=A^{-(\alpha+\beta)} y$, o que, por sua vez, significa que $x \in R\left(A^{-(\alpha+\beta)}\right)=D\left(A^{\alpha+\beta}\right) \mathrm{e}$ $A^{\alpha+\beta} x=A^{\alpha} A^{\beta} x$.

E, se $x \in D\left(A^{\alpha+\beta}\right)$ e $A^{\alpha+\beta} x=y$, segue que $x=A^{-(\alpha+\beta)} y=A^{-\alpha} A^{-\beta} y=A^{-\beta} A^{-\alpha} y$, ou seja, 
$x \in R\left(A^{-\beta}\right)=D\left(A^{\beta}\right)$ e $A^{\beta} x \in R\left(A^{-\alpha}\right)=D\left(A^{\alpha}\right)$, donde $x \in D\left(A^{\alpha} A^{\beta}\right)$.

Por fim, $A^{\alpha} A^{\beta}=A^{\alpha+\beta}=A^{\beta+\alpha}=A^{\beta} A^{\alpha}$ em $D\left(A^{\gamma}\right)$, onde $\gamma=\operatorname{máx}\{\alpha, \beta, \alpha+\beta\}$.

Caso 2: $\alpha, \beta<0$.

Segue do último Teorema 1.2.5.

Caso 3: $\alpha>0, \beta=-\gamma, \gamma>0, \operatorname{com} \alpha>\gamma$.

Para provar que $A^{\alpha} A^{\beta}=A^{\alpha+\beta}$, observemos que isso é equivalente a verificar que $\left(A^{\alpha} A^{-\gamma}\right)\left(A^{-(\alpha-\gamma)}\right)=$ I. Agora, usando o Teorema 1.2.5, temos que $\left(A^{\alpha} A^{-\gamma}\right)\left(A^{-(\alpha-\gamma)}\right)=A^{\alpha}\left(A^{-\gamma} A^{\gamma-\alpha}\right)=A^{\alpha} A^{-\alpha}=I$. Analogamente, mostramos que $A^{\beta} A^{\alpha}=A^{\alpha+\beta}$.

Caso 4: $\alpha>0, \beta=-\gamma, \gamma>0, \operatorname{com} \alpha<\gamma$.

Novamente, como no caso anterior, basta demonstrarmos que $A^{\alpha} A^{-\gamma}=A^{\alpha-\gamma}$ e que $A^{-\gamma} A^{\alpha}=$ $A^{\alpha-\gamma}$. Aplicando o Caso $1, A^{-(\alpha-\gamma)} A^{\alpha} A^{-\gamma}=A^{\gamma} A^{-\gamma}=I$ e $A^{-\gamma} A^{\alpha} A^{-(\alpha-\gamma)}=A^{-\gamma} A^{\gamma}=I$.

Agora, estamos prontos para iniciar a prova do Teorema 1.2.9 em questão:

\section{Demonstração do Teorema 1.2.9:}

A partir das estimativas obtidas no Teorema 1.1.9 e das propriedades no Lema 1.2.10, temos para $\alpha=m=1,2,3 \ldots$,

$$
\begin{aligned}
\left\|A^{m} e^{-A t}\right\| & =\left\|\left(A e^{-A \frac{t}{m}}\right)^{m}\right\| \\
& \leq\left\|A e^{-A \frac{t}{m}}\right\|^{m} \\
& \leq\left(C_{2} m\right)^{m} t^{-m} e^{-\delta t} .
\end{aligned}
$$


Se $0<\alpha<1$ e $t>0$,

$$
\begin{aligned}
\left\|A^{\alpha} e^{-A t}\right\| & =\left\|A A^{-(1-\alpha)} e^{-A t}\right\| \\
& =\left\|A e^{-A t} A^{-(1-\alpha)}\right\| \\
& \leq \frac{1}{\Gamma(1-\alpha)} \int_{0}^{\infty} s^{-\alpha}\left\|A e^{-A(t+s)}\right\| d s \\
& \leq \frac{1}{\Gamma(1-\alpha)} \int_{0}^{\infty} s^{-\alpha}(t+s)^{-1} C_{2} e^{-\delta(t+s)} d s \\
& \leq \frac{C_{2} e^{-\delta t}}{\Gamma(1-\alpha)} \int_{0}^{\infty} s^{-\alpha}(s+t)^{-1} d s \\
& \leq \frac{C_{2} e^{-\delta t}}{\Gamma(1-\alpha)} \frac{\pi}{\sin (\pi \alpha)} t^{-\alpha} \\
& =\frac{C_{2} e^{-\delta t}}{\Gamma(1-\alpha)} \Gamma(\alpha) \Gamma(1-\alpha) t^{-\alpha} \\
& =C_{2} \Gamma(\alpha) t^{-\alpha} e^{-\delta t},
\end{aligned}
$$

onde aplicamos os Teoremas 1.1.9 1 1.2.5 e o Lema 1.2.10.

E, para $\alpha \in \mathbb{Z} \backslash\{0\}$ ou $\in(0,1)$ e $\beta \in \mathbb{Z} \backslash\{0\}$ ou $\in(0,1)$, reunindo os casos anteriores,

$$
\begin{aligned}
\left\|A^{\alpha+\beta} e^{-A t}\right\| & =\left\|A^{\alpha} A^{\beta} e^{-A \frac{t}{2}} e^{-A \frac{t}{2}}\right\| \\
& =\left\|A^{\alpha} e^{-A \frac{t}{2}} A^{\beta} e^{-A \frac{t}{2}}\right\| \\
& \leq\left\|A^{\alpha} e^{-A \frac{t}{2}}\right\|\left\|A^{\beta} e^{-A \frac{t}{2}}\right\| \\
& \leq C_{\alpha}\left(\frac{t}{2}\right)^{-\alpha} e^{-\delta \frac{t}{2}} C_{\beta}\left(\frac{t}{2}\right)^{-\beta} e^{-\delta \frac{t}{2}} \\
& =C_{\alpha} C_{\beta} 2^{\alpha+\beta} t^{-(\alpha+\beta)} e^{-\delta t} .
\end{aligned}
$$

Notemos que o caso geral é uma combinação destes afinal, podemos escrever um número qualquer $\alpha$ como a soma de um natural e um número entre 0 e 1 . 
Para a outra estimativa, usando o Lema 1.2.10 e a primeira parte, se $0<\alpha \leq 1$ e $x \in D\left(A^{\alpha}\right)$,

$$
\begin{aligned}
\left\|e^{-A t} x-x\right\| & =\left\|-\int_{0}^{t} A e^{-A s} x d s\right\| \\
& =\left\|-\int_{0}^{t} A^{(1-\alpha)+\alpha} e^{-A s} x d s\right\| \\
& =\left\|-\int_{0}^{t} A^{1-\alpha} A^{\alpha} e^{-A s} x d s\right\| \\
& =\left\|-\int_{0}^{t} A^{1-\alpha} e^{-A s} A^{\alpha} x d s\right\| \\
& \leq \int_{0}^{t}\left\|A^{1-\alpha} e^{-A s}\right\|\left\|A^{\alpha} x\right\| d s \\
& \leq \int_{0}^{t} C_{1-\alpha} s^{-(1-\alpha)} e^{-\delta s}\left\|A^{\alpha} x\right\| d s \\
& \leq C_{1-\alpha}\left\|A^{\alpha} x\right\| \int_{0}^{t} s^{-(1-\alpha)} d s \\
& =\frac{1}{\alpha} C_{1-\alpha} t^{\alpha}\left\|A^{\alpha} x\right\| .
\end{aligned}
$$

Finalmente, com relação a afirmação sobre $C_{\alpha}$, pelo que fizemos, $C_{\alpha}=C_{2} \Gamma(\alpha), 0<\alpha<1$, $C_{\alpha}=\left(C_{2} m\right)^{m}, \alpha=m=1,2, \ldots$, ou $C_{\alpha}=C_{m} C_{\beta} 2^{m+\beta}$, se $\alpha=m+\beta>1$, onde $m \in \mathbb{N}^{*}, 0<\beta<1$, de modo que se $\alpha$ está em um compacto de $(0, \infty)$, ou seja, um intervalo fechado e limitado, $C_{\alpha}$ fica também limitada. E é importante observarmos que como tal constante $C_{\alpha}$ é basicamente uma potência daquela constante $C_{2}$ do Teorema 1.1 .9 multiplicada por um número derivado de $\alpha, C_{\alpha}$ depende de $M$ e do setor para $A$.

Teorema 1.2.11 Se $0 \leq \alpha \leq 1$ e $x \in D(A)$, então $\left\|A^{\alpha} x\right\| \leq C\|A x\|^{\alpha}\|x\|^{1-\alpha}$, o que implica que $\left\|A^{\alpha} x\right\| \leq \alpha \epsilon\|A x\|+(1-\alpha) C^{\frac{1}{1-\alpha}} \epsilon^{\frac{-\alpha}{(1-\alpha)}}\|x\|, \forall \epsilon>0$, onde $C>0$ é uma constante independente de $\alpha$ dada por:

$$
C=\frac{4 C_{1}}{\left(\frac{1}{e}\right)^{\frac{1}{e}}}
$$

sendo $C_{1}$ a constante do Teorema 1.1 .9

\section{Demonstração:}


Sejam $0<\beta<1$ e $\epsilon>0$,

$$
\begin{aligned}
\left\|\Gamma(\beta) A^{-\beta} x\right\| & =\left\|\int_{0}^{\epsilon} t^{\beta-1} e^{-A t} x d t+\int_{\epsilon}^{\infty} t^{\beta-1} e^{-A t} x d t\right\| \\
& \leq \int_{0}^{\epsilon} t^{\beta-1}\left\|e^{-A t} x\right\| d t+\left\|\int_{\epsilon}^{\infty} t^{\beta-1} e^{-A t} x d t\right\| \\
& \leq C_{1}\|x\| \int_{0}^{\epsilon} t^{\beta-1} d t+\left\|\epsilon^{\beta-1} e^{-A \epsilon} A^{-1} x\right\|+\left\|(\beta-1) \int_{\epsilon}^{\infty} t^{\beta-2} e^{-A t} A^{-1} x d t\right\| \\
& \leq C_{1} \frac{\epsilon^{\beta}}{\beta}\|x\|+C_{1} \epsilon^{\beta-1}\left\|A^{-1} x\right\|+(1-\beta) \int_{\epsilon}^{\infty} t^{\beta-2} C_{1}\left\|A^{-1} x\right\| d t \\
& =C_{1} \frac{\epsilon^{\beta}}{\beta}\|x\|+C_{1} \epsilon^{\beta-1}\left\|A^{-1} x\right\|+C_{1} \epsilon^{\beta-1}\left\|A^{-1} x\right\| \\
& =C_{1} \frac{\epsilon^{\beta}}{\beta}\|x\|+2 C_{1} \epsilon^{\beta-1}\left\|A^{-1} x\right\|,
\end{aligned}
$$

onde $C_{1}$ é a constante do Teorema 1.1.9. Denotemos por $f(\epsilon)$ o lado direito desta desigualdade, $f(\epsilon)=C_{1} \frac{\epsilon^{\beta}}{\beta}\|x\|+2 C_{1} \epsilon^{\beta-1}\left\|A^{-1} x\right\|, \epsilon>0$. Observemos que $\lim _{\epsilon \rightarrow 0} f(\epsilon)=+\infty$ e $\lim _{\epsilon \rightarrow+\infty} f(\epsilon)=$ $+\infty$, de modo que podemos a seguir determinar seu mínimo:

$$
\begin{aligned}
f^{\prime}(\epsilon) & =0 \\
& \Leftrightarrow \quad C_{1} \epsilon^{\beta-1}\|x\|+2 C_{1}(\beta-1) \epsilon^{\beta-2}\left\|A^{-1} x\right\|=0 \\
& \Leftrightarrow \quad C_{1} \epsilon^{\beta-2}\left(\epsilon\|x\|+2(\beta-1)\left\|A^{-1} x\right\|\right)=0 \\
& \Leftrightarrow \quad \epsilon=\frac{2(1-\beta)\left\|A^{-1} x\right\|}{\|x\|}, x \neq 0 .
\end{aligned}
$$

E, o mínimo de $f$ ocorre no ponto $\epsilon=\frac{2(1-\beta)\left\|A^{-1} x\right\|}{\|x\|}>0$. Substituindo na expressão (1.9):

$$
\begin{aligned}
\left\|\Gamma(\beta) A^{-\beta} x\right\| & \leq \frac{C_{1}\|x\|}{\beta}\left(\frac{2(1-\beta)\left\|A^{-1} x\right\|}{\|x\|}\right)^{\beta}+2 C_{1}\left(\frac{2(1-\beta)\left\|A^{-1} x\right\|}{\|x\|}\right)^{\beta-1}\left\|A^{-1} x\right\| \\
& =C_{1}\|x\|^{1-\beta}\left\|A^{-1} x\right\|^{\beta}\left(\frac{2^{\beta}(1-\beta)^{\beta}}{\beta}+2^{\beta}(1-\beta)^{\beta-1}\right)
\end{aligned}
$$

donde,

$$
\begin{aligned}
\left\|A^{-\beta} x\right\| & \leq C_{1}\|x\|^{1-\beta}\left\|A^{-1} x\right\|^{\beta}\left(\frac{(2(1-\beta))^{\beta-1}(2(1-\beta)+2 \beta)}{\beta \Gamma(\beta)}\right) \\
& =C_{1}\|x\|^{1-\beta}\left\|A^{-1} x\right\|^{\beta}\left(\frac{2(2(1-\beta))^{\beta-1}}{\Gamma(1+\beta)}\right) .
\end{aligned}
$$

Ponhamos então $x=A x$ e $\alpha=1-\beta$, obtendo:

$$
\left\|A^{\alpha} x\right\| \leq C_{1}\|A x\|^{\alpha}\|x\|^{1-\alpha}\left(\frac{2(2 \alpha)^{-\alpha}}{\Gamma(2-\alpha)}\right) .
$$

Agora, se $0<\beta<1$, podemos limitar o coeficiente uniformemente nesse intervalo. Com efeito, 
(i) $\Gamma(1+\beta) \geq \frac{1}{2}$.

(ii) $2^{1-\beta} \geq 1$.

(iii) Resta estudar $(1-\beta)^{1-\beta}$ :

Seja $g(x)=(1-x)^{1-x}, 0<x<1$ (vide Figura 1.15).

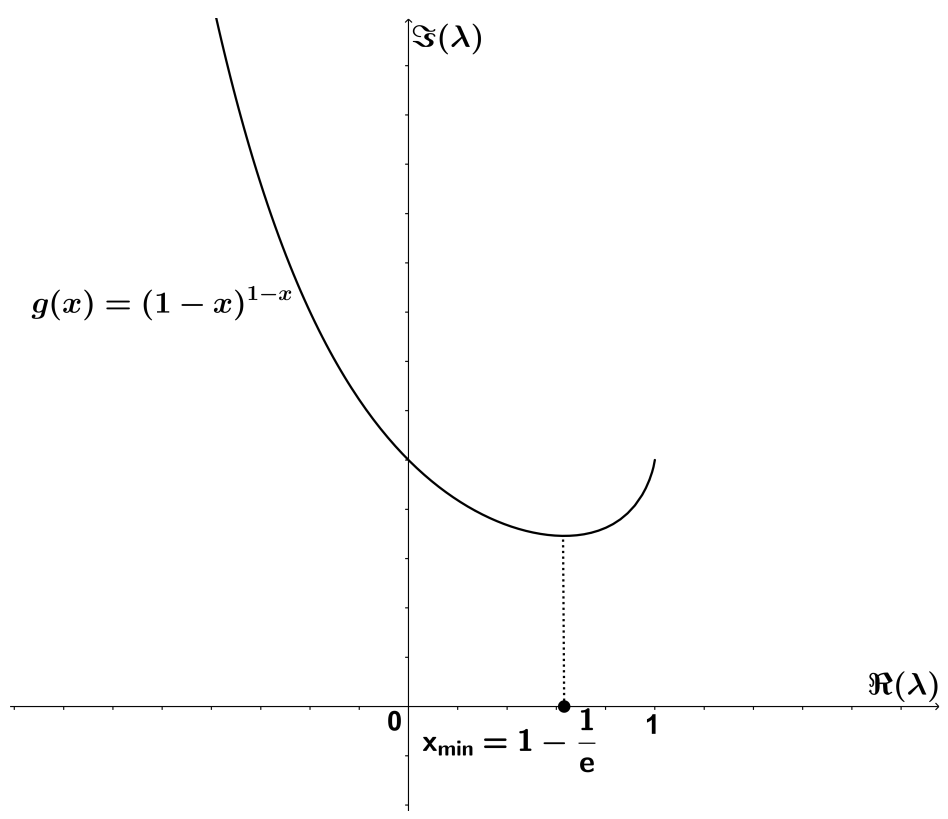

Figura 1.15: Gráfico da função g.

Então, $g^{\prime}(x)=\left(e^{(1-x) \log (1-x)}\right)^{\prime}=(1-x)^{-x}(x-1)(\log (1-x)+1) \mathrm{e}$,

$$
\begin{aligned}
g^{\prime}(x) & =0 \\
& \Leftrightarrow \quad(1-x)^{-x}(x-1) \log (1-x)+(1-x)^{-x}(x-1)=0 \\
& \Leftrightarrow \log (1-x)=-1 \\
& \Leftrightarrow \quad e^{\log (1-x)}=\frac{1}{e} \\
& \Leftrightarrow 1-x=\frac{1}{e} \\
& \Leftrightarrow x=1-\frac{1}{e} .
\end{aligned}
$$

E, $g^{\prime \prime}(x)=(1-x)^{-x}\left(-x-(x-1) \log ^{2}(1-x)-2(x-1) \log (1-x)+2\right)$, o que nos permite concluir que $g(x) \geq g\left(1-\frac{1}{e}\right)=\left(\frac{1}{e}\right)^{\frac{1}{e}}$ afinal, $g^{\prime \prime}\left(1-\frac{1}{e}\right)=2 e^{\left(1-\frac{1}{e}\right)}>0$. 
Reunindo essas considerações, temos

$$
\left(\frac{2(2(1-\beta))^{\beta-1}}{\Gamma(1+\beta)}\right) \leq \frac{4}{\left(\frac{1}{\epsilon}\right)^{\frac{1}{\epsilon}}} .
$$

Logo, para $0<\alpha<1,\left\|A^{\alpha} x\right\| \leq C\|A x\|^{\alpha}\|x\|^{1-\alpha}$, onde $C$ depende exclusivamente de $M$ e do setor para A afinal, $C$ é $C_{1}$ do Teorema 1.1 .9 multiplicado por um número independente de $\alpha$. Os casos $\alpha=0$ e $\alpha=1$ seguem trivialmente.

Para obter a outra expressão, precisaremos da seguinte desigualdade auxiliar:

Desigualdade de Young: Se $p$ e $q$ são números reais positivos tais que $\frac{1}{p}+\frac{1}{q}=1$, então para todo par $a, b$ de números reais não negativos:

$$
a b \leq \frac{a^{p}}{p}+\frac{b^{q}}{q} .
$$

Notemos que $\left\|A^{\alpha} x\right\| \leq C\|A x\|^{\alpha}\|x\|^{1-\alpha}$ pode ser reescrito como $\left\|A^{\alpha} x\right\| \leq \epsilon^{\alpha}\|A x\|^{\alpha} C \epsilon^{-\alpha} \|$ $x \|^{1-\alpha}, \forall \epsilon>0$. Assim, tomando $a=\epsilon^{\alpha}\|A x\|^{\alpha}, b=C \epsilon^{-\alpha}\|x\|^{1-\alpha}, p=\frac{1}{\alpha}, q=\frac{1}{1-\alpha}$ e aplicando tal desigualdade, encontramos o desejado:

$$
\left\|A^{\alpha} x\right\| \leq \alpha \epsilon\|A x\|+(1-\alpha) C^{\frac{1}{1-\alpha}} \epsilon^{\frac{-\alpha}{1-\alpha}}\|x\| .
$$

Observação 1.2.12 Se $\left\|e^{-A t}\right\| \leq C_{1} e^{-\delta t} e\left\|A e^{-A t}\right\| \leq C_{2} t^{-1} e^{-\delta t}, t>0$, então, usando os Teoremas 1.1.9 e 1.2.11,

$$
\begin{aligned}
\left\|A^{\alpha} e^{-A t} x\right\| & \leq C\left\|A e^{-A t} x\right\|^{\alpha}\left\|e^{-A t} x\right\|^{1-\alpha} \\
& \leq C\left(C_{2} t^{-1} e^{-\delta t}\right)^{\alpha}\left(C_{1} e^{-\delta t}\right)^{1-\alpha}\|x\| \\
& =C\left(C_{2}\right)^{\alpha}\left(C_{1}\right)^{1-\alpha} t^{-\alpha} e^{-\delta t}\|x\| .
\end{aligned}
$$

E, essa última expressão converge para $C C_{1} e^{-\delta t}\|x\|$, quando $\alpha$ tende a $0^{+}$, lembrando que $C$ é uma constante independente de $\alpha$. Isso prova que a constante $C_{\alpha}$ no Teorema 1.2.9 é limitada quando $\alpha$ converge $a 0^{+}$.

Corolário 1.2.13 Se A é um operador setorial com $\mathfrak{R}(\sigma(A))>0$ e B é um operador linear fechado tal que $B A^{-\alpha}$ é limitado em X para algum $0 \leq \alpha<1$, então $A+B$ é setorial.

\section{Demonstração:}

Nosso objetivo a seguir é verificar as hipóteses do Exemplo 1.1.4: 
(i) Como A é um operador setorial com $\mathfrak{R}(\sigma(A))>0$, podemos considerar um setor $S_{0, \phi_{0}}$, para algum $\phi_{0} \in\left(0, \frac{\pi}{2}\right)$ apropriado, empregando a notação da Definição 1.1.1.E, ainda,

$$
\begin{aligned}
\left\|A(\lambda I-A)^{-1}\right\| & =\left\|\lambda(\lambda I-A)^{-1}-(\lambda I-A)(\lambda I-A)^{-1}\right\| \\
& \leq|\lambda|\left\|(\lambda I-A)^{-1}\right\|+1 \\
& \leq|\lambda| \frac{M}{|\lambda|}+1 \\
& =M+1=C, \forall \lambda \in S_{0, \phi_{0}} .
\end{aligned}
$$

Em particular, essa desigualdade é válida para $\lambda \in S_{0, \phi_{0}} \operatorname{com}|\lambda| \geq R_{0}$, qualquer $R_{0}>0$.

(ii) O operador $B$ é linear, fechado e $D(B) \supset D(A)$, pois o fato de $B A^{-\alpha}$ ser limitado significa que o domínio deste operador é todo $\mathrm{X}, D(B) \supset R\left(A^{-\alpha}\right)=D\left(A^{\alpha}\right)$ e, por sua vez, $D\left(A^{\alpha}\right) \supset D(A)$ para $0 \leq \alpha<1$ pelo Lema 1.2.10. Além disso, usando o Teorema 1.2.11, $\forall x \in D(A)$ e $\forall \epsilon>0$, temos:

$$
\begin{aligned}
\|B x\| & =\left\|B A^{-\alpha} A^{\alpha} x\right\| \\
& \leq\left\|B A^{-\alpha}\right\|\left\|A^{\alpha} x\right\| \\
& \leq\left\|B A^{-\alpha}\right\|\left(\alpha \epsilon\|A x\|+(1-\alpha) C^{\frac{1}{1-\alpha}} \epsilon^{\frac{-\alpha}{(1-\alpha)}}\|x\|\right) \\
& =\left\|B A^{-\alpha}\right\| \alpha \epsilon\|A x\|+\left\|B A^{-\alpha}\right\|(1-\alpha) C^{\frac{1}{1-\alpha}} \epsilon^{\frac{-\alpha}{(1-\alpha)}}\|x\| .
\end{aligned}
$$

Então, se tomarmos $\epsilon$ tal que $C\left\|B A^{-\alpha}\right\| \alpha \epsilon<1$, isto é, $\epsilon<\frac{1}{C \alpha\left\|B A^{-\alpha}\right\|}$, o resultado segue do Exemplo 1.1.4.

Teorema 1.2.14 Sejam A e B operadores setoriais em $X \operatorname{com} D(A)=D(B), \mathfrak{R}(\sigma(A))>0, \mathfrak{R}(\sigma(B))>0$, para algum $\alpha \in[0,1),(A-B) A^{-\alpha}$ é limitado em $X$. Então, dado qualquer $\beta \in[0,1], A^{\beta} B^{-\beta}$ e $B^{\beta} A^{-\beta}$ são limitados em $X$.

\section{Demonstração:}

Conforme discutimos na demonstração do Teorema 1.1.9 podemos supor que o setor para $A$ tem vértice $a=0$ e abertura $\phi \in\left(0, \frac{\pi}{2}\right)$ de maneira a termos a seguinte situação para $-A$ (vide Figura 1.16): 


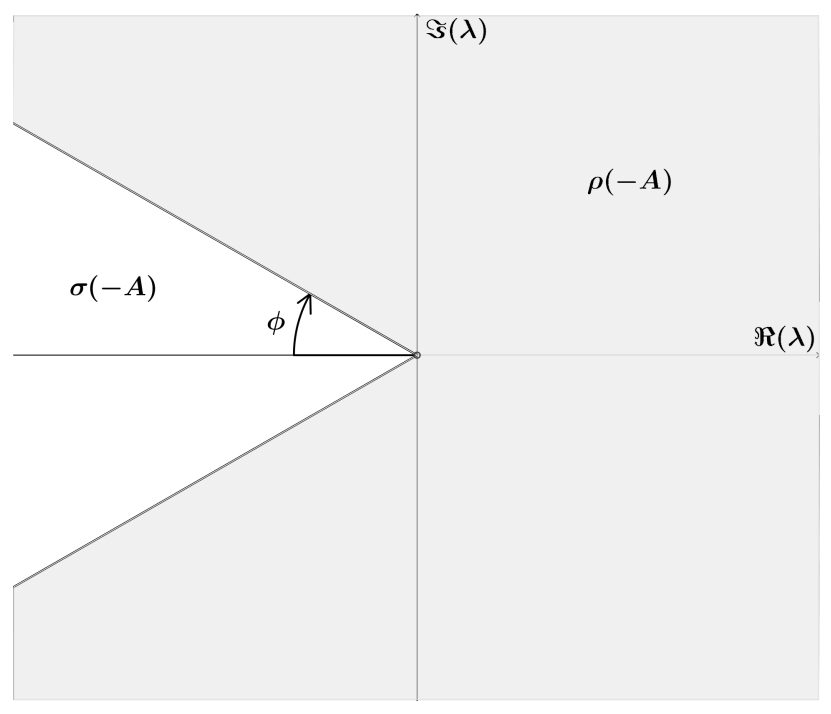

Figura 1.16: Situação para -A.

(i) Para $0 \leq \beta \leq 1,|\pi-\arg (\lambda)| \geq \phi$ e $\phi<\frac{\pi}{2}$,

$$
\begin{aligned}
\left\|A^{\beta}(-\lambda I-A)^{-1}\right\| & =\sup _{\|x\|=1}\left\|A^{\beta}(\lambda I+A)^{-1} x\right\| \\
& \leq \sup _{\|x\|=1}\left(C\left\|A(\lambda I+A)^{-1} x\right\|^{\beta}\left\|(\lambda I+A)^{-1} x\right\|^{1-\beta}\right) \\
& \leq \sup _{\|x\|=1}\left(C(1+M)^{\beta}\|x\|^{\beta}\left(\frac{M}{|\lambda|}\right)^{1-\beta}\|x\|^{1-\beta}\right) \\
& =C(1+M)^{\beta} M^{1-\beta}|\lambda|^{\beta-1},
\end{aligned}
$$

onde $C$ é a constante do Teorema 1.2.11 e aplicamos a desigualdade (1.5). Logo, $\left\|A^{\beta}(\lambda I+A)^{-1}\right\| \leq$ $C_{A, \beta}|\lambda|^{\beta-1}$, sendo $C_{A, \beta}=C(1+M)^{\beta} M^{1-\beta}$, constante dependente de $A$ e de $\beta$.

(ii) Também podemos majorar tal norma do seguinte modo:

$$
\begin{aligned}
\left\|A^{\beta}(\lambda I+A)^{-1}\right\| & =\left\|A^{\beta-1} A(\lambda I+A)^{-1}\right\| \\
& \leq\left\|A^{\beta-1}\right\|\left\|A(\lambda I+A)^{-1}\right\|,
\end{aligned}
$$

ou seja, $\left\|A^{\beta}(\lambda I+A)^{-1}\right\| \leq K_{A, \beta}$ afinal, ambas as normas na expressão (1.10) são limitadas por constantes de acordo com o Teorema 1.2.5e a desigualdade (1.5).

(iii) Observemos que cálculos análogos a esses podem ser feitos com o operador $B^{\beta}(\lambda I+B)^{-1}$.

Além disso, se $0<\beta<1$, aplicando uma das identidades do resolvente,

$$
\begin{aligned}
B^{-\beta}-A^{-\beta} & =\frac{\sin (\pi \beta)}{\pi} \int_{0}^{\infty} \lambda^{-\beta}(\lambda I+B)^{-1} d \lambda-\frac{\sin (\pi \beta)}{\pi} \int_{0}^{\infty} \lambda^{-\beta}(\lambda I+A)^{-1} d \lambda \\
& =\frac{\sin (\pi \beta)}{\pi}\left(\int_{0}^{\infty} \lambda^{-\beta}\left((\lambda I+B)^{-1}-(\lambda I+A)^{-1}\right) d \lambda\right) \\
& =\frac{\sin (\pi \beta)}{\pi} \int_{0}^{\infty} \lambda^{-\beta}(\lambda I+B)^{-1}(A-B)(\lambda I+A)^{-1} d \lambda
\end{aligned}
$$


Agora, $-B^{\beta} A^{-\beta}=B^{\beta}\left(B^{-\beta}-A^{-\beta}\right)-I$ de modo que para mostrar que o operador $B^{\beta} A^{-\beta}$ é limitado, basta limitar $B^{\beta}\left(B^{-\beta}-A^{-\beta}\right)$. Para $\alpha \in[0,1)$ tal que $(A-B) A^{-\alpha}$ é limitado, usando as considerações em (i) à (iii),

$$
\begin{aligned}
\left\|B^{\beta}\left(B^{-\beta}-A^{-\beta}\right)\right\| & =\left\|B^{\beta} \frac{\sin (\pi \beta)}{\pi} \int_{0}^{\infty} \lambda^{-\beta}(\lambda I+B)^{-1}(A-B)(\lambda I+A)^{-1} d \lambda\right\| \\
& =\left\|\frac{\sin (\pi \beta)}{\pi} \int_{0}^{\infty} \lambda^{-\beta} B^{\beta}(\lambda I+B)^{-1}(A-B)(\lambda I+A)^{-1} d \lambda\right\| \\
& =\left\|\frac{\sin (\pi \beta)}{\pi} \int_{0}^{\infty} \lambda^{-\beta} B^{\beta}(\lambda I+B)^{-1}(A-B) A^{-\alpha} A^{\alpha}(\lambda I+A)^{-1} d \lambda\right\| \\
& \leq\left\|\frac{\sin (\pi \beta)}{\pi} \int_{0}^{1} \lambda^{-\beta} B^{\beta}(\lambda I+B)^{-1}(A-B) A^{-\alpha} A^{\alpha}(\lambda I+A)^{-1} d \lambda\right\| \\
& +\left\|\frac{\sin (\pi \beta)}{\pi} \int_{1}^{\infty} \lambda^{-\beta} B^{\beta}(\lambda I+B)^{-1}(A-B) A^{-\alpha} A^{\alpha}(\lambda I+A)^{-1} d \lambda\right\| \\
& \leq \frac{|\sin (\pi \beta)|}{\pi} \int_{0}^{1}|\lambda|^{-\beta}\left\|B^{\beta}(\lambda I+B)^{-1}\right\|\left\|(A-B) A^{-\alpha}\right\|\left\|A^{\alpha}(\lambda I+A)^{-1}\right\| d \lambda \\
& +\frac{|\sin (\pi \beta)|}{\pi} \int_{1}^{\infty}|\lambda|^{-\beta}\left\|B^{\beta}(\lambda I+B)^{-1}\right\|\left\|(A-B) A^{-\alpha}\right\|\left\|A^{\alpha}(\lambda I+A)^{-1}\right\| d \lambda \\
& \leq \frac{|\sin (\pi \beta)|}{\pi} \int_{0}^{1}|\lambda|^{-\beta} K_{B, \beta}\left\|(A-B) A^{-\alpha}\right\| K_{A, \alpha} d \lambda \\
& +\frac{|\sin (\pi \beta)|}{\pi} \int_{1}^{\infty}|\lambda|^{-\beta} C_{B, \beta}|\lambda|^{\beta-1}\left\|(A-B) A^{-\alpha}\right\| C_{A, \alpha}|\lambda|^{\alpha-1} d \lambda \\
& =\frac{|\sin (\pi \beta)|}{\pi} K_{B, \beta}\left\|(A-B) A^{-\alpha}\right\| K_{A, \alpha} \int_{0}^{1}|\lambda|^{-\beta} d \lambda \\
& +\frac{|\sin (\pi \beta)|}{\pi} C_{B, \beta} C_{A, \alpha}\left\|(A-B) A^{-\alpha}\right\| \int_{1}^{\infty}|\lambda|^{\alpha-2} d \lambda .
\end{aligned}
$$

Assim, constatamos que $\left\|B^{\beta}\left(B^{-\beta}-A^{-\beta}\right)\right\|$ é finita dado que $0<\beta<1$ e $0 \leq \alpha<1$ garantem a convergência das integrais em (1.11) e (1.12) respectivamente.

Para o outro caso, temos semelhantemente:

$$
A^{-\beta}-B^{-\beta}=\frac{\sin (\pi \beta)}{\pi} \int_{0}^{\infty} \lambda^{-\beta}(\lambda I+A)^{-1}(B-A) A^{-\alpha} A^{\alpha}(\lambda I+B)^{-1} d \lambda .
$$

E, notamos que:

(i) $(A-B) A^{-\alpha}$ limitado implica que $(B-A) A^{-\alpha}$ também o é.

(ii) $-A^{\beta} B^{-\beta}=A^{\beta}\left(A^{-\beta}-B^{-\beta}\right)-I$, sendo suficiente limitar $A^{\beta}\left(A^{-\beta}-B^{-\beta}\right)$. Para tanto, as majorações seriam essencialmente as mesmas que fizemos acima, exceto pelo último termo como podemos 
observar. Vejamos:

$$
\begin{aligned}
\left(I+A^{\alpha}(\lambda I+A)^{-1}(B-A) A^{-\alpha}\right) A^{\alpha}(\lambda I+B)^{-1} & =A^{\alpha}(\lambda I+B)^{-1}+A^{\alpha}(\lambda I+A)^{-1}(B-A)(\lambda I+B)^{-1} \\
& =A^{\alpha}(\lambda I+B)^{-1}+A^{\alpha}\left((\lambda I+A)^{-1}-(\lambda I+B)^{-1}\right) \\
& =A^{\alpha}(\lambda I+A)^{-1}
\end{aligned}
$$

o que signifca que,

$$
\left\|\left(I+A^{\alpha}(\lambda I+A)^{-1}(B-A) A^{-\alpha}\right) A^{\alpha}(\lambda I+B)^{-1}\right\|=\left\|A^{\alpha}(\lambda I+A)^{-1}\right\|,
$$

isto é, $\left\|A^{\alpha}(\lambda I+B)^{-1}\right\|=O\left(\left\|A^{\alpha}(\lambda I+A)^{-1}\right\|\right)$ afinal, $A^{\alpha}(\lambda I+A)^{-1}$ e $(B-A) A^{-\alpha}$ são operadores limitados.

Daí, bastaria aplicar considerações análogas para concluir que $A^{\beta} B^{-\beta}$ também é limitado.

Por fim, falta analisar os casos $\beta=0$ e $\beta=1$.

Se $\beta=0, A^{\beta} B^{-\beta}=I=B^{\beta} A^{-\beta}$.

E, se $\beta=1$, então $A B^{-1}$ e $B A^{-1}$ são limitados, pois podemos escrever, por exemplo, $I-B A^{-1}=$ $(A-B) A^{-1}=(A-B) A^{-\alpha} A^{\alpha-1}, \operatorname{com}(A-B) A^{-\alpha}$ limitado para algum $\alpha \in[0,1)$ por hipótese e, como justamente, $\alpha-1<0, A^{\alpha-1}$ também é limitado.

Teorema 1.2.15 Sejam A um operador setorial em um espaço de Banach $X$ com $\mathfrak{R}(\sigma(A))>\delta>0$ e $B$ um operador linear de $D(B) \subset X$ em um espaço de Banach $Y$. Suponha que $D(B) \supset D(A)$ e, para algum $0 \leq \alpha<1$ e alguma constante $C>0$,

$$
\|B x\|_{Y} \leq C\|A x\|^{\alpha}\|x\|^{1-\alpha}, \forall x \in D(A),
$$

ou equivalentemente,

$$
\|B x\|_{Y} \leq \varepsilon\|A x\|+K \varepsilon^{\frac{-\alpha}{(1-\alpha)}}\|x\|, \forall \varepsilon>0 e \forall x \in D(A),
$$

para alguma constante $K>0$.

Então, para qualquer $\beta$ com $\alpha<\beta \leq 1, B$ tem uma única extensão linear contínua de $X^{\beta}$ a $Y$ (ver Definição 1.2.17 abaixo), isto é, $B A^{-\beta}$ é um operador contínuo.

Para demonstrar esse Teorema, precisaremos da seguinte propriedade:

Lema 1.2.16 Seja A um operador setorial em um espaço de Banach $X$. Se $\alpha \geq \beta \geq 0$, então $X^{\alpha}$ é denso em $X^{\beta}$ (com a norma $\left.\beta\right)$. 


\section{Demonstração:}

De fato, sabemos pelo Lema 1.2.10 que $X^{\gamma}=D\left(A_{1}^{\gamma}\right)$ é denso em $X, \forall \gamma \geq 0$. Considere $x \in X^{\beta}$, então $A_{1}^{\beta} x \in X$. Seja $y_{n} \in X^{\alpha-\beta}$ tal que $y_{n} \rightarrow A_{1}^{\beta} x$ em $X$.

Segue que $A_{1}^{-\beta} y_{n} \in X^{\alpha} \mathrm{e}$,

$$
\left\|A_{1}^{-\beta} y_{n}-x\right\|_{\beta}=\left\|A_{1}^{\beta}\left(A_{1}^{-\beta} y_{n}-x\right)\right\|=\left\|y_{n}-A_{1}^{\beta} x\right\| \rightarrow 0,
$$

ou seja, $A_{1}^{-\beta} y_{n} \rightarrow x$ em $X^{\beta}$.

\section{Demonstração do Teorema 1.2.15:}

Seja $x \in X$, então $A^{-1} x \in D(A) \subset D(B)$ e, por hipótese,

$$
\begin{aligned}
\left\|B A^{-1} x\right\|_{Y} & \leq C\left\|A A^{-1} x\right\|^{\alpha}\left\|A^{-1} x\right\|^{1-\alpha} \\
& \leq C\|x\|^{\alpha}\left\|A^{-1}\right\|^{1-\alpha}\|x\|^{1-\alpha} \\
& =C\left\|A^{-1}\right\|^{1-\alpha}\|x\|,
\end{aligned}
$$

donde $B A^{-1}$ é um operador limitado em $\mathrm{X}$.

Agora, para $x \in D\left(A^{\beta+1}\right)$, podemos escrever:

$$
\begin{aligned}
B x & =B A^{-1} A A^{\beta} A^{-\beta} x \\
& =B A^{-1} A A^{\beta} \frac{1}{\Gamma(\beta)} \int_{0}^{\infty} t^{\beta-1} e^{-A t} x d t \\
& =B A^{-1} A \frac{1}{\Gamma(\beta)} \int_{0}^{\infty} t^{\beta-1} e^{-A t} A^{\beta} x d t \\
& =B A^{-1} \frac{1}{\Gamma(\beta)} \int_{0}^{\infty} t^{\beta-1} e^{-A t} A^{\beta+1} x d t \\
& =\frac{1}{\Gamma(\beta)} \int_{0}^{\infty} t^{\beta-1} B e^{-A t} A^{\beta} x d t .
\end{aligned}
$$

Vejamos que a função do lado direito, $x \in X^{\beta} \mapsto \frac{1}{\Gamma(\beta)} \int_{0}^{\infty} t^{\beta-1} B e^{-A t} A^{\beta} x d t \in Y$, é contínua,

$$
\begin{aligned}
\left\|\frac{1}{\Gamma(\beta)} \int_{0}^{\infty} t^{\beta-1} B e^{-A t} A^{\beta} x d t\right\| & \leq \frac{1}{\Gamma(\beta)} \int_{0}^{\infty} t^{\beta-1}\left\|B e^{-A t} A^{\beta} x\right\| d t \\
& \leq \frac{1}{\Gamma(\beta)} \int_{0}^{\infty} t^{\beta-1} C\left\|A e^{-A t} A^{\beta} x\right\|^{\alpha}\left\|e^{-A t} A^{\beta} x\right\|^{1-\alpha} d t \\
& \leq \frac{1}{\Gamma(\beta)} \int_{0}^{\infty} t^{\beta-1} C\left(\left\|A e^{-A t}\right\|\left\|A^{\beta} x\right\|\right)^{\alpha}\left(\left\|e^{-A t}\right\|\left\|A^{\beta} x\right\|\right)^{1-\alpha} d t \\
& \leq \frac{1}{\Gamma(\beta)} \int_{0}^{\infty} t^{\beta-1} C\left(C_{2} t^{-1} e^{-\delta t}\right)^{\alpha}\left\|A^{\beta} x\right\|^{\alpha}\left(C_{1} e^{-\delta t}\right)^{1-\alpha}\left\|A^{\beta} x\right\|^{1-\alpha} d t \\
& =\frac{1}{\Gamma(\beta)} C\left(C_{2}\right)^{\alpha}\left(C_{1}\right)^{1-\alpha} \int_{0}^{\infty} t^{(\beta-\alpha)-1} e^{-\delta t} d t\left\|A^{\beta} x\right\|,
\end{aligned}
$$


sendo $C_{1}$ e $C_{2}$ as constantes do Teorema 1.1.9. Notemos que, na última expressão, a exponencial garante a convergência no infinito, enquanto que o fato de $1>\beta>\alpha$ garante a convergência da integral no zero. Logo, B admite uma extensão linear contínua de $X^{\beta}$ em $Y$. E, ela é única devido a $X^{\beta+1}$ ser denso em $X^{\beta}$, conforme Lema 1.2.16.

Definição 1.2.17 Se A é um operador setorial em um espaço de Banach X, defina para cada $\alpha \geq 0$,

$$
\begin{gathered}
X^{\alpha}=D\left(A_{1}^{\alpha}\right), \text { com a norma do gráfico, } \\
\|x\|_{\alpha}=\left\|A_{1}^{\alpha} x\right\|, x \in X^{\alpha}
\end{gathered}
$$

onde $A_{1}=A+$ aI, com a escolhido tal que $\mathfrak{R}\left(\sigma\left(A_{1}\right)\right)>0$.

É importante notarmos que diferentes escolhas de $a$ dão origem a normas equivalentes:

Com efeito, sejam $A_{1}=A+a_{1}$ e $A_{2}=A+a_{2}$, sendo $a_{1}$ e $a_{2}$ tais que $\mathfrak{R}\left(\sigma\left(A_{1}\right)\right)>0$ e $\mathfrak{R}\left(\sigma\left(A_{2}\right)\right)>0$. Podemos supor que $a_{1}<a_{2}$. Temos assim $A_{1}$ e $A_{2}$ operadores setoriais em $X \operatorname{com} D\left(A_{1}\right)=D\left(A_{2}\right)=$ $D(A)$ e $\left(A_{2}-A_{1}\right) A_{2}^{-\alpha}=\left(a_{2}-a_{1}\right) A_{2}^{-\alpha}$ limitado em $X$ para todo $\alpha \in[0,1)$, em particular, $\alpha=0$. Pelo Teorema 1.2.14, $A_{1}^{\beta} A_{2}^{-\beta}$ e $A_{2}^{\beta} A_{1}^{-\beta}$ são limitados em $\mathrm{X}$ para todo $\beta \in[0,1]$. Ou seja, para $\alpha \in[0,1]$ as normas dos espaços $X_{1}^{\alpha}=D\left(A_{1}^{\alpha}\right)$ e $X_{2}^{\alpha}=D\left(A_{2}^{\alpha}\right)$ são equivalentes. E, se $\alpha>1$ e $\alpha=m$, $m$ inteiro:

$$
\begin{aligned}
\left\|A_{2}^{m} x\right\| & =\left\|A_{2} A_{2}^{m-1} x\right\| \\
& \leq C\left\|A_{1} A_{2}^{m-1} x\right\| \\
& =C\left\|A_{2}^{m-1} A_{1} x\right\| \\
& \leq C^{2}\left\|A_{1} A_{2}^{m-2} A_{1} x\right\| \\
& \cdot \\
& \cdot \\
& \cdot \\
& \leq C^{m}\left\|A_{1}^{m} x\right\|,
\end{aligned}
$$

onde C é uma constante relativa a equivalência de normas para $\alpha=1$ e aplicamos indução para obter a última expressão. Devemos, porém, justificar uma passagem essencial nestes cálculos:

Sabemos que $A_{1}$ e $A_{2}$ comutam e, a partir disso, precisamos mostrar que $A_{1}$ e $A_{2}^{\beta}$ comutam, $\operatorname{com} \beta>0$.

Dados dois operadores $C$ e $D$ que comutam e são inversíveis, então $C$ comuta $\operatorname{com} D^{-1}$ e vice-versa, pois:

$$
C D=D C \Leftrightarrow C=D^{-1} C D \Leftrightarrow C D^{-1}=D^{-1} C,
$$


de modo que basta verificar que $A_{1} \operatorname{comuta} \operatorname{com} A_{2}^{-\beta}$.

Pela Definição 1.2.1.

$$
A_{2}^{-\beta}=\frac{1}{\Gamma(\beta)} \int_{0}^{\infty} t^{\beta-1} e^{-A_{2} t} d t
$$

lembrando que,

$$
e^{-A_{2} t}=\frac{1}{2 \pi i} \int_{\Gamma}\left(\lambda I+A_{2}\right)^{-1} e^{\lambda t} d \lambda
$$

sendo $\Gamma$ como no Teorema 1.1.9.

Como $A_{1}$ comuta com o operador $\lambda I+A_{2}, \forall \lambda \in \Gamma, A_{1}$ comuta com o inverso desse e resulta assim que $A_{1}$ comuta com $A_{2}^{-\beta}, \beta>0$.

Por fim, para $\alpha>1$ qualquer, podemos escrever $\alpha=m+\beta$, com $m \in \mathbb{N}^{*}, \beta \in(0,1)$ e, reunindo os casos anteriores, temos

$$
\begin{aligned}
\left\|A_{2}^{m+\beta} x\right\| & =\left\|A_{2}^{m} A_{2}^{\beta} x\right\| \\
& \leq C^{m}\left\|A_{1}^{m} A_{2}^{\beta} x\right\| \\
& =C^{m}\left\|A_{2}^{\beta} A_{1}^{m} x\right\| \\
& \leq C^{m} \tilde{C}\left\|A_{1}^{\beta} A_{1}^{m} x\right\|,
\end{aligned}
$$

onde $\tilde{C}$ é a constante referente a equivalência de normas para o índice $\beta$.

Teorema 1.2.18 Se Aé um operador setorial em um espaço de Banach X, então $X^{\alpha}$ é um espaço de Banach com a norma $\|\cdot\|_{\alpha}$, para $\alpha \geq 0, X^{0}=X$, e para $\alpha \geq \beta \geq 0, X^{\alpha}$ é um subespaço denso de $X^{\beta}$ com inclusão contínua. Se A tem resolvente compacto, a inclusão $X^{\alpha} \subset X^{\beta}$ é compacta quando $\alpha>\beta \geq 0$.

Além disso, se $A_{1}$ e $A_{2}$ são dois operadores setoriais com mesmo domínio, $\mathfrak{R}\left(\sigma\left(A_{j}\right)\right)>0, j=1,2$ e, $\left(A_{1}-A_{2}\right) A_{1}^{-\alpha}$ é limitado para algum $\alpha<1$, então se $X_{j}^{\beta}=D\left(A_{j}^{\beta}\right),(j=1,2), X_{1}^{\beta}=X_{2}^{\beta}$ com normas equivalentes para $0 \leq \beta \leq 1$ e esta equivalência depende apenas dos setores e das constantes $M_{1}$ e $M_{2}$ na Definição 1.1.1 associados à $A_{1}$ e $A_{2}$.

\section{Demonstração:}

Em etapas:

(i) $\left(X^{\alpha},\|\|_{\alpha}\right)$ é um espaço de Banach, $\alpha \geq 0$.

Seja $\left(x_{n}\right)_{n \in \mathbb{N}}$ uma sequência de Cauchy em $X^{\alpha}$, isto é, dado $\varepsilon>0$, existe $n_{0} \in \mathbb{N}$ tal que $\left\|x_{n}-x_{m}\right\|_{\alpha} \leq \varepsilon, \forall n, m \geq n_{0}$.

Como $\left\|x_{n}-x_{m}\right\|_{\alpha}:=\left\|A_{1}^{\alpha}\left(x_{n}-x_{m}\right)\right\|_{X}=\left\|A_{1}^{\alpha} x_{n}-A_{1}^{\alpha} x_{m}\right\|_{X}$, segue que a sequência $\left(A_{1}^{\alpha} x_{n}\right)_{n \in \mathbb{N}}$ é de Cauchy em $X$. Sendo este um espaço completo, temos que $\left(A_{1}^{\alpha} x_{n}\right)_{n \in \mathbb{N}}$ converge para algum $y \in X$ 
quando $n \rightarrow \infty$.

Agora, $A_{1}^{-\alpha}$ é um operador limitado conforme vimos no Teorema 1.2.5, donde $x_{n}=A_{1}^{-\alpha}\left(A_{1}^{\alpha} x_{n}\right) \rightarrow$ $A_{1}^{-\alpha} y \operatorname{em~X}$ quando $n \rightarrow \infty$. Notemos que $x=A_{1}^{-\alpha} y \in X^{\alpha} \mathrm{e},\left\|x_{n}-x\right\|_{\alpha}=\left\|A_{1}^{\alpha}\left(x_{n}-x\right)\right\|_{X}=\left\|A_{1}^{\alpha} x_{n}-y\right\|_{X}$ que converge a zero quando $n \rightarrow \infty$. Logo, $\left(x_{n}\right)_{n \in \mathbb{N}}$ é uma sequência convergente e, pela sua arbitrariedade, podemos concluir que $X^{\alpha}$ é um espaço de Banach com a norma $\alpha, \alpha \geq 0$.

(ii) Para $\alpha \geq \beta \geq 0$, $X^{\alpha}$ é um subespaço denso de $X^{\beta}$ com inclusão contínua.

Já sabemos pelo Lema 1.2.10 que para $\alpha \geq \beta \geq 0, X^{\alpha} \subset X^{\beta}$. E a questão da densidade, provamos no Lema 1.2.16. Resta mostrar que a inclusão $X^{\alpha} \subset X^{\beta}$ é contínua. Usando o referido Lema 1.2.10, se $x \in X^{\alpha}$,

$$
\begin{aligned}
\|x\|_{\beta} & =\left\|A_{1}^{\beta} x\right\| \\
& =\left\|A_{1}^{\alpha} A_{1}^{-\alpha} A_{1}^{\beta} x\right\| \\
& =\left\|A_{1}^{\alpha} A_{1}^{-(\alpha-\beta)} x\right\| \\
& =\left\|A_{1}^{-(\alpha-\beta)} A_{1}^{\alpha} x\right\| \\
& \leq\left\|A_{1}^{-(\alpha-\beta)}\right\|\left\|A_{1}^{\alpha} x\right\| \\
& =\left\|A_{1}^{-(\alpha-\beta)}\right\|\|x\|_{\alpha} .
\end{aligned}
$$

(iii) Se $A$ tem resolvente compacto, a inclusão $X^{\alpha} \subset X^{\beta}$ é compacta quando $\alpha>\beta \geq 0$.

Inicialmente, vale recordar que um operador $A$ tem resolvente compacto se existir $\lambda \in \rho(A)$ tal que $(\lambda I-A)^{-1}$ é um operador compacto e, se existir, o mesmo é válido para todo $\lambda \in \rho(A)$.

Consideremos a inclusão $i=\left.A^{-\beta} \circ \bar{i} \circ A^{\beta}\right|_{X^{\alpha}}: X^{\alpha} \rightarrow X^{\beta}$, onde $A^{-\beta}: X \rightarrow X^{\beta}, \bar{i}: X^{\alpha-\beta} \hookrightarrow X \mathrm{e}$ $\left.A^{\beta}\right|_{X^{\alpha}}: X^{\alpha} \rightarrow X^{\alpha-\beta}$. Vimos no Teorema 1.2.5 que $A^{-\beta}$ é um operador contínuo e, como para todo $x \in X^{\alpha}$, vale:

$$
\left\|A^{\beta} x\right\|_{\alpha-\beta}:=\left\|A^{\alpha-\beta} A^{\beta} x\right\|=\left\|A^{\alpha} x\right\|=\|x\|_{\alpha}
$$

segue que $\left.A^{\beta}\right|_{X^{\alpha}}$ também é contínuo.

É suficiente, dessa forma, provar que $\bar{i}$ é compacta, ou seja, se $B_{\alpha-\beta}$ é a bola de centro na origem e raio um de $X^{\alpha-\beta}$, então $\bar{i}\left(B_{\alpha-\beta}\right)$ está contida em um conjunto compacto de $X$. Como

$$
B_{\alpha-\beta}=\left\{x \in X^{\alpha-\beta} \mid\|x\|_{\alpha-\beta} \leq 1\right\}=\left\{y \in X \mid\|y\| \leq 1, y \in R\left(A^{\alpha-\beta}\right)\right\}=A^{-(\alpha-\beta)}\left(B_{0}\right),
$$

significa que reduzimos o nosso problema a mostrar que $A^{-\gamma}$ é um operador compacto, $\forall \gamma \geq 0$, sabendo que A tem resolvente compacto. 
Para $0<\gamma<1$,

$$
A^{-\gamma}=\frac{\sin (\pi \gamma)}{\pi} \int_{0}^{\infty} \lambda^{-\gamma}(\lambda I+A)^{-1} d \lambda
$$

e temos o limite de somas finitas de operadores compactos que é um operador compacto, lembrando que $(\lambda I+A)^{-1}$ é compacto por hipótese, $\forall \lambda \in(0,+\infty) \subset \rho(-A)$. Para as demais potências, basta usar composição afinal, a composição de operadores compactos é compacta.

(iv) Se $A_{1}$ e $A_{2}$ são dois operadores setoriais com mesmo domínio, $\mathfrak{R}\left(\sigma\left(A_{j}\right)\right)>0, j=1,2$ e, $\left(A_{1}-A_{2}\right) A_{1}^{-\alpha}$ é limitado para algum $\alpha<1$, então se $X_{j}^{\beta}=D\left(A_{j}^{\beta}\right),(j=1,2), X_{1}^{\beta}=X_{2}^{\beta}$ com normas equivalentes para $0 \leq \beta \leq 1$ e esta equivalência depende apenas dos setores e das constantes $M_{1}$ e $M_{2}$ na Definição 1.1.1 associados à $A_{1}$ e $A_{2}$.

Finalmente, para esta última parte, a equivalência das normas em $X_{1}^{\beta}$ e $X_{2}^{\beta}$ e o fato desta equivalência depender dos setores e das constantes $M_{1}$ e $M_{2}$ para $A_{1}$ e $A_{2}$ vem do Teorema 1.2.14. Precisamos apenas demonstrar que tais espaços são de fato iguais.

Observemos que podemos escrever $A_{1}^{\beta}$ como $A_{1}^{\beta} A_{2}^{-\beta} A_{2}^{\beta}$ e, sendo $A_{1}^{\beta} A_{2}^{-\beta}$ um operador limitado, temos que $X_{1}^{\beta}=D\left(A_{1}^{\beta}\right)=D\left(A_{1}^{\beta} A_{2}^{-\beta} A_{2}^{\beta}\right)=D\left(A_{2}^{\beta}\right)=X_{2}^{\beta}$.

\subsection{Um teorema de imersão}

Iniciamos exibindo o conceito da propriedade de extensão $C^{m}$ e as Desigualdades de Nirenberg - Gagliardo. O objetivo é demonstrar um importante resultado sobre os espaços $X^{\alpha}$, com o qual finalizamos a seção.

Definição 1.3.1 Seja $\Omega \subset \mathbb{R}^{n}$. Dizemos que $\Omega$ tem a propriedade da extensão $C^{m}$ se existe uma extensão $E: C_{c}^{m}(\bar{\Omega}) \rightarrow C_{c}^{m}\left(\mathbb{R}^{n}\right)$ de modo que $E(\phi)$ restrita a $\bar{\Omega}$ é $\phi$ e para as normas de quaisquer dos espaços $C^{v}$ ou $W^{k, q}(0 \leq v, k \leq m, 1 \leq q<\infty)$, existe uma constante $B>0$ com,

$$
B^{-1}\|\phi\|_{\Omega} \leq\|E(\phi)\|_{R^{n}} \leq B\|\phi\|_{\Omega}
$$

onde $\|\cdot\|_{\Omega}$ denota a norma em $C^{v}(\Omega)$ ou $W^{k, q}(\Omega)$ e $\|\cdot\|_{\mathbb{R}^{n}}$, a norma em $C^{v}\left(\mathbb{R}^{n}\right)$ ou $W^{k, q}\left(\mathbb{R}^{n}\right)$.

Seja $\Omega \subset \mathbb{R}^{n}$ com a propriedade da extensão $C^{m}$,

Proposição 1.3.2 Uma versão das Desigualdades de Nirenberg - Gagliardo: 
(a) $\|u\|_{W^{k, p}(\Omega)} \leq C\|u\|_{W^{m, q}(\Omega)}^{\theta}\|u\|_{L_{r}(\Omega)^{\prime}}^{1-\theta}$ se $p \geq q, p \geq r, 0 \leq \theta \leq 1 e k-\frac{n}{p} \leq \theta\left(m-\frac{n}{q}\right)-\frac{n}{r}(1-\theta)$, sendo a desigualdade estrita se $q$ ou $r=1$.

(b) $\|u\|_{C^{v}(\Omega)} \leq C\|u\|_{W^{m, q}(\Omega)}^{\theta}\|u\|_{L_{r}(\Omega)^{\prime}}^{1-\theta}$ se $0 \leq \theta \leq 1 e v \leq \theta\left(m-\frac{n}{q}\right)-\frac{n}{r}(1-\theta)$,

sendo a desigualdade estrita se q ou $r=1$, ou se vé inteiro.

Teorema 1.3.3 Suponha que $\Omega \subset \mathbb{R}^{n}$ é um aberto com a propriedade da extensão $C^{m}$ e A é um operador setorial em $X=L_{p}(\Omega), 1 \leq p<\infty, \operatorname{com} D(A)=X^{1} \subset W^{m, p}(\Omega)$ para algum $m \geq 1$. Então, para $0 \leq \alpha \leq 1$

$$
\begin{gathered}
X^{\alpha} \subset W^{k, q}(\Omega), \text { quando } k-\frac{n}{q}<m \alpha-\frac{n}{p}, q \geq p, \\
X^{\alpha} \subset C^{v}(\Omega), \text { quando } 0 \leq v<m \alpha-\frac{n}{p} .
\end{gathered}
$$

\section{Demonstração:}

Pela Desigualdade de Nirenberg - Gagliardo (a),

$$
\|u\|_{W^{k, q(\Omega)}} \leq C\|u\|_{W^{m, p}(\Omega)}^{\theta}\|u\|_{L_{p}(\Omega)^{\prime}}^{1-\theta}
$$

desde que $q \geq p, k-\frac{n}{q} \leq \theta\left(m-\frac{n}{p}\right)-\frac{n}{p}(1-\theta)=m \theta-\frac{n}{p}$. Para $u \in D(A)$,

$$
\|u\|_{W^{k, q}(\Omega)} \leq \tilde{C}\|A u\|_{L_{p}(\Omega)}^{\theta}\|u\|_{L_{p}(\Omega)}^{1-\theta} .
$$

Note que acima consideramos $\mathfrak{R}(\sigma(A))>0$, o que é possível pois, a rigor, estamos trabalhando com os espaços $X^{\alpha}$, os quais são definidos a partir do operador $A_{1}=A+a$, $\operatorname{com} a$ tal que $\mathfrak{R}\left(\sigma\left(A_{1}\right)\right)>0$.

Seja i a inclusão $i: D(A) \subset W^{m, p}(\Omega) \hookrightarrow W^{k, q}(\Omega)$. Aplicando o Teorema1.2.15, para todo $\alpha$, $\theta<\alpha \leq 1, i$ possui uma única extensão linear contínua de $X^{\alpha}$ para $W^{k, q}(\Omega)$, ou seja,

$$
X^{\alpha} \subset W^{k, q}(\Omega) \text {, onde } k-\frac{n}{q}<m \alpha-\frac{n}{p}, q \geq p .
$$

Para a segunda afirmação, usando a outra desigualdade de Nirenberg - Gagliardo (b),

$$
\|u\|_{C^{v}(\Omega)} \leq C\|u\|_{W^{m, p}(\Omega)}^{\theta}\|u\|_{L_{p}(\Omega)^{\prime}}^{1-\theta} \text {, se } 0 \leq \theta \leq 1 \text { e } v \leq \theta\left(m-\frac{n}{p}\right)-\frac{n}{p}(1-\theta)=m \theta-\frac{n}{p} .
$$

Analogamente ao que fizemos na primeira parte, temos para $u \in D(A)$ :

$$
\|u\|_{C^{v}(\Omega)} \leq \tilde{C}\|A u\|_{L_{p}(\Omega)}^{\theta}\|u\|_{L_{p}(\Omega)}^{1-\theta} .
$$

Novamente, de acordo com o Teorema 1.2.15, a inclusão $i: D(A) \subset W^{m, p}(\Omega) \hookrightarrow C^{v}(\Omega)$ possui uma única extensão linear contínua de $X^{\alpha}$ para $C^{v}(\Omega)$, sempre que $\theta<\alpha \leq 1$ :

$$
X^{\alpha} \subset C^{v}(\Omega) \text {, onde } 0 \leq v<m \alpha-\frac{n}{p} .
$$




\subsection{Uma importante aplicação}

Nesta seção, utilizamos a bagagem obtida conjuntamente com resultados demonstrados em Henry (1981) para garantir uma limitação de certo modo uniforme em $X^{\beta}$ das soluções de problemas de Cauchy para uma família de equações não lineares envolvendo um operador setorial.

Consideremos a equação não linear:

$$
\left\{\begin{array}{l}
\frac{d x}{d t}+A x=f(t, x), t>t_{0} \\
x\left(t_{0}\right)=x_{0}
\end{array}\right.
$$

onde A é um operador setorial tal que as potências fracionárias de $A_{1} \equiv A+a ; a$ de forma que $\mathfrak{R}\left(\sigma\left(A_{1}\right)\right)>0$; estão bem definidas e, os espaços $X^{\alpha}=D\left(A_{1}^{\alpha}\right)$ com a norma do gráfico $\|x\|_{\alpha}=\left\|A_{1}^{\alpha} x\right\|$ são definidos para $\alpha \geq 0$. Suponha que $f: U \subset \mathbb{R} \times X^{\alpha} \rightarrow X, U$ um conjunto aberto, $0 \leq \alpha<1$ e $f$ é localmente Holder contínua em te localmente Lipschitz em $x$ pertencentes a $U$. Mais precisamente, se $\left(t_{1}, x_{1}\right) \in U$, existe uma vizinhança $V \subset U$ de $\left(t_{1}, x_{1}\right)$, tal que para $(t, x),(s, y) \in V$,

$$
\|f(t, x)-f(s, y)\| \leq L\left(|t-s|^{\theta}+\|x-y\|_{\alpha}\right),
$$

para constantes $L>0$ e $\theta>0$.

Definição 1.4.1 Uma solução do problema de Cauchy (1.13) (ou problema de valor inicial) em $\left(t_{0}, t_{1}\right)$ é uma função contínua $x:\left[t_{0}, t_{1}\right) \rightarrow X$ tal que $x\left(t_{0}\right)=x_{0} e$, em $\left(t_{0}, t_{1}\right)$, temos $(t, x(t)) \in U, x(t) \in D(A)$, existe $\frac{d x}{d t}$, a aplicação $t \rightarrow f(t, x(t))$ é localmente Holder contínua, $\int_{t_{0}}^{t_{0}+\rho}\|f(t, x(t))\| d t<\infty$ para algum $\rho>0, \int_{t_{0}}^{t}(t-s)^{-\alpha}\|f(s, x(s))\| d s \rightarrow 0$ quando $t \rightarrow t_{0}^{+} e$, a equação diferencial é satisfeita em $\left(t_{0}, t_{1}\right)$.

Teorema 1.4.2 Sejam A um operador setorial e $f: U \rightarrow X, U$ um subconjunto aberto de $\mathbb{R} \times X^{\alpha}, 0 \leq \alpha<1$, fé localmente Holder contínua em te localmente Lipschitziana em $x$. Então, para qualquer $\left(t_{0}, x_{0}\right) \in U$, existe $T=T\left(t_{0}, x_{0}\right)>0$ tal que a equação diferencial (1.13) tem uma única solução $x$ em $\left(t_{0}, t_{0}+T\right)$ com valor inicial $x\left(t_{0}\right)=x_{0}$.

Demonstração: Veja o Teorema 3.3.3 em Henry (1981).

Teorema 1.4.3 Sejam A e $f$ como no enunciado do Teorema 1.4.2 e suponhamos também que A tem resolvente compacto e $f$ aplica todos os conjuntos da forma $\mathbb{R}^{+} \times B \subset U \subset \mathbb{R} \times X^{\alpha}$, com $B$ fechado $e$ limitado, em conjuntos limitados de $X$. Se $x\left(t ; t_{0}, x_{0}\right)$ é a solução da equação diferencial $(1.13)$ em $\left(t_{0}, \infty\right)$ com $\left\|x\left(t ; t_{0}, x_{0}\right)\right\|_{\alpha}$ permanecendo limitada à medida que $t \rightarrow+\infty$, então $\left\{x\left(t ; t_{0}, x_{0}\right)\right\}_{t>t_{0}}$ está em um conjunto compacto em $X^{\alpha}$. 
Demonstração: Veja o Teorema 3.3.6 em Henry (1981).

Essencialmente, o que se faz para demonstrar este resultado é provar que $\left\|x\left(t ; t_{0}, x_{0}\right)\right\|_{\beta}$ é limitado para $t \geq t_{0}+1, \alpha<\beta<1$ afinal, de acordo com o Teorema 1.2.18, a inclusão $X^{\beta} \subset X^{\alpha}$ é compacta. E, obtém-se a seguinte limitação,

$$
\left\|x\left(t ; t_{0}, x_{0}\right)\right\|_{\beta} \leq C_{\beta-\alpha}\left(t-t_{0}\right)^{-(\beta-\alpha)} e^{-\delta\left(t-t_{0}\right)}\left\|x_{0}\right\|_{\alpha}+\int_{t_{0}}^{t} C_{\beta}(t-s)^{-\beta} e^{-\delta(t-s)} C d s,
$$

onde $C$ é tal que $\left\|f\left(t, x\left(t ; t_{0}, x_{0}\right)\right)\right\| \leq C, \forall t \geq t_{0}$ e, $C_{\beta-\alpha}$ e $C_{\beta}$ são as constantes determinadas no Teorema 1.2.9 que, como vimos, dependem da constante $M$ e do setor relativos ao operador A. Essa observação será de grande relevância para o nosso estudo.

Uma aplicação interessante desse resultado pode ser feita no contexto do seguinte problema abstrato:

$$
\left\{\begin{array}{l}
\frac{d x}{d t}+A_{\gamma} x=f_{\gamma}(x), t>t_{0} \\
x\left(t_{0}\right)=x_{0} \in X_{\gamma}^{\alpha},
\end{array}\right.
$$

onde $\left\{A_{\gamma}\right\}_{\gamma \in \Lambda}$ é uma família de operadores setoriais em $X_{\gamma}^{\alpha}, 0 \leq \alpha<1$, com resolvente compacto, $D\left(A_{\gamma}\right) \supset D(A), \forall \gamma \in \Lambda$, sendo $\Lambda$ um espaço topológico e $A=A_{\gamma_{0}}, f_{\gamma}: U \rightarrow X, U$ um subconjunto aberto de $X_{\gamma}^{\alpha}$, localmente Lipschitziana em x e $f_{\gamma}$ aplica todos os subconjuntos fechados e limitados de $U$ em subconjuntos limitados de $X$. Nesse contexto, o Teorema 1.4.2 nos garante a existência e a unicidade de solução localmente.

Suponhamos que as soluções desses problemas estejam globalmente definidas e denotemos por $T_{\gamma}\left(t, x_{0}\right)$ o respectivo semigrupo gerado.

Proposição 1.4.4 Se $T_{\gamma}\left(t, x_{0}\right)$ admite um atrator global $\mathcal{A}_{\gamma}$ e este é limitado em $X_{\gamma}^{\alpha}$ para determinado $0 \leq \alpha<1$, então tal conjunto é também limitado em $X_{\gamma}^{\beta} \operatorname{com} \alpha<\beta<1$.

\section{Demonstração:}

De fato, recordemos que $\mathcal{A}_{\gamma}=\left\{x_{0} \in X_{\gamma}^{\alpha} \mid\right.$ existe uma solução global limitada por $\left.x_{0}\right\}$.

As soluções de (1.15) estando globalmente definidas, podemos aplicar o Teorema1.4.3 e temos que $\left\{x\left(t ; t_{0}, x_{0}\right)\right\}_{t>t_{0}}$ é limitado em $X_{\gamma}^{\beta}$, onde $\alpha<\beta<1$, conforme a expressão (1.14).

Como $\|x\|_{X_{\gamma}^{\alpha}} \leq R_{\gamma}, \forall x \in \mathcal{A}_{\gamma}$, para alguma constante $R_{\gamma}>0$, tal limitação é uniforme em $x_{0}$. Em outras palavras, $\left\{T_{\gamma}(t) x_{0}\right\}_{t>t_{0}}$ é limitada em $X_{\gamma}^{\beta}$ uniformemente em $x_{0}$.

Agora, o conjunto $\mathcal{A}_{\gamma}$ é invariante sob a ação de $T_{\gamma}\left(t, x_{0}\right)$, isto é, $T_{\gamma}\left(t, x_{0}\right) \mathcal{A}_{\gamma}=\mathcal{A}_{\gamma}, \forall t \geq t_{0}$, em particular, $T_{\gamma}\left(t_{0}+1, x_{0}\right) \mathcal{A}_{\gamma}=\mathcal{A}_{\gamma}$.

Logo, concluimos que $\|x\|_{X_{\gamma}^{\beta}} \leq S_{\gamma}, \forall x \in \mathcal{A}_{\gamma}$, para alguma constante $S_{\gamma}>0$. 
Nosso objetivo a seguir é estudar tal constante $S_{\gamma}$ obtida da limitação do atrator $\mathcal{A}_{\gamma}$ em $X_{\gamma}^{\beta}$, mais precisamente, em quais condições $S_{\gamma}$ independe do parâmetro $\gamma ; S_{\gamma}=S$. Vejamos:

De acordo com a expressão (1.14):

$$
\begin{aligned}
\left\|x\left(t_{0}+1 ; t_{0}, x_{0}\right)\right\|_{X_{\gamma}^{\beta}} & \leq C_{\gamma, \beta-\alpha}(1)^{-(\beta-\alpha)} e^{-\delta} R_{\gamma}+\int_{t_{0}}^{t_{0}+1} C_{\gamma, \beta}\left(t_{0}+1-s\right)^{-\beta} e^{-\delta\left(t_{0}+1-s\right)} C_{\gamma} d s \\
& \leq C_{\gamma, \beta-\alpha} R_{\gamma}+C_{\gamma, \beta} C_{\gamma} \int_{t_{0}}^{t_{0}+1}\left(t_{0}+1-s\right)^{-\beta} d s \\
& \leq C_{\gamma, \beta-\alpha} R_{\gamma}+C_{\gamma, \beta} C_{\gamma}
\end{aligned}
$$

onde $C_{\gamma, \beta-\alpha}=\left(C_{2}\right)_{\gamma} \Gamma(\beta-\alpha), C_{\gamma, \beta}=\left(C_{2}\right)_{\gamma} \Gamma(\beta)$ e $R_{\gamma}$ e $C_{\gamma}$ vem respectivamente de $\|x\|_{X_{\gamma}^{\alpha}} \leq R_{\gamma}, \forall x \in$ $\mathcal{A}_{\gamma}$ e $\left\|f_{\gamma}\left(x\left(t ; t_{0}, x_{0}\right)\right)\right\| \leq C_{\gamma}, \forall t \geq t_{0}$, sendo $\left(C_{2}\right)_{\gamma}$ a constante do Teorema 1.1 .9 referente ao operador $A_{\gamma}$.

Assim, se a família $\left\{A_{\gamma}\right\}_{\gamma \in \Lambda}$ for tal que é possível estabelecer um setor e uma constante $M$ comuns a todos os seus elementos e aquelas constantes $R_{\gamma}$ e $C_{\gamma}$ também não dependerem de $\gamma$, a limitação obtida para o atrator em $X_{\gamma}^{\beta}$ é uniforme em $\gamma$, ou seja, $S_{\gamma}$ independe de $\gamma, S_{\gamma}=S$.

Nessa situação, parece conveniente podermos comparar as normas em $X_{\gamma}^{\beta}$ e $X^{\beta}=X_{\gamma_{0}}^{\beta}$. Para isso, faremos novas suposições sobre os operadores $A_{\gamma}$ :

Teorema 1.4.5 Seja $\left\{A_{\gamma}\right\}_{\gamma \in \Lambda}$ uma família de operadores positivos definidos e autoadjuntos em um espaço de Hilbert $X$ com $\Lambda$ um espaço topológico e $A=A_{\gamma_{0}}$, satisfazendo as seguintes condições:

(i) $D\left(A_{\gamma}\right) \subset X$ densa e continuamente $\forall \gamma \in \Lambda$, istoé, $\|x\| \leq L\left\|A_{\gamma} x\right\|, \forall x \in D\left(A_{\gamma}\right)$, onde Lé uma constante;

(ii) $D(A) \subset D\left(A_{\gamma}\right), \forall \gamma \in \Lambda$;

(iii) $\left\|\left(A-A_{\gamma}\right) x\right\| \leq \epsilon(\gamma)\|A x\|+K(\gamma)\|x\|, \forall x \in D(A)$, onde $\epsilon(\gamma), K(\gamma) \rightarrow 0$ quando $\gamma \rightarrow \gamma_{0}$.

Então, $\|x\|_{X_{\gamma}^{\beta}} \leq K_{1}(\gamma)\|x\|_{X^{\beta}}$ e, analogamente, $\|x\|_{X^{\beta}} \leq K_{2}(\gamma)\|x\|_{X_{\gamma}^{\beta}}$, com $K_{1}(\gamma), K_{2}(\gamma) \rightarrow 1$ quando $\gamma \rightarrow \gamma_{0}$, uniformemente em $\beta$.

\section{Demonstração:}

Sabendo que $X_{\gamma}=X$ é um espaço de Hilbert e que os operadores $A_{\gamma}$ são positivos definidos e autoadjuntos para todo $\gamma \in \Lambda$, segue de resultados conhecidos que os domínios de suas potências fracionárias de ordem $\beta$ coincidem isometricamente com os espaços de interpolação, ou seja, $\left[X, X_{\gamma}^{1}\right]_{\beta}=D\left(A_{\gamma}^{\beta}\right)$ e $\left[X, X^{1}\right]_{\beta}=D\left(A^{\beta}\right)$ com isometrias, para qualquer $0<\beta<1$ (Veja o Teorema 16.1 em Yagi (2010), por exemplo).

E se $X_{\gamma}^{1} \subset X$ densa e continuamente, $\forall \gamma \in \Lambda$, isto é, $\|x\| \leq L\left\|A_{\gamma} x\right\|, \forall x \in X_{\gamma}^{1}$ sendo 
L uma constante, então, para qualquer $0<\beta<1$, (Teorema 1.15 em Yagi (2010)),

$$
\begin{aligned}
\|I\|_{L\left(\left[X, X_{\gamma}^{1}\right]_{\beta},\left[X, X^{1}\right]_{\beta}\right)} & \leq\|I\|_{L(X, X)}^{1-\beta}\|I\|_{L\left(X_{\gamma}^{1}, X^{1}\right)}^{\beta} \\
& \leq\|I\|_{L\left(X_{\gamma}^{1}, X^{1}\right)^{\prime}}^{\beta}
\end{aligned}
$$

onde $I: Y \rightarrow Z$ denota o operador inclusão de $Y \subset Z$ em $Z$.

Além disso, a partir da desigualdade,

$$
\left\|\left(A-A_{\gamma}\right) x\right\| \leq \epsilon(\gamma)\|A x\|+K(\gamma)\|x\|, \forall x \in X^{1},
$$

onde $\epsilon(\gamma)$ e $K(\gamma)$ tendem a 0 à medida que $\gamma \rightarrow \gamma_{0}$, vale que,

$$
\|A x\| \leq \tau(\gamma)\left\|A_{\gamma} x\right\|,
$$

sendo $\tau(\gamma)=\frac{1+K(\gamma) l}{1-\epsilon(\gamma)}$ que converge a 1 quando $\gamma \rightarrow \gamma_{0}$. Daí,

$$
\|I x\|_{X^{1}}=\|x\|_{D(A)}=\|A x\| \leq \tau(\gamma)\left\|A_{\gamma} x\right\|=\tau(\gamma)\|x\|_{D\left(A_{\gamma}\right)}=\tau(\gamma)\|x\|_{X_{\gamma}^{1}} .
$$

Consequentemente, $\|I\|_{L\left(X_{\gamma}^{1}, X^{1}\right)} \leq \tau(\gamma)$ e aplicando isso na desigualdade (1.16), encontramos,

$$
\|x\|_{\left[X, X^{1}\right]_{\beta}} \leq \tau(\gamma)^{\beta}\|x\|_{\left[X, X_{\gamma}^{1}\right]_{\beta}} .
$$

Ou ainda,

$$
\|x\|_{X^{\beta}} \leq K_{2}(\gamma)\|x\|_{X_{\gamma}^{\beta}}
$$

com $K_{2}(\gamma) \rightarrow 1$ quando $\gamma \rightarrow \gamma_{0}$, uniformemente em $\beta$.

Analogamente obtemos a desigualdade reversa, $\|x\|_{X_{\gamma}^{\beta}} \leq K_{1}(\gamma)\|x\|_{X^{\beta}}, K_{1}(\gamma) \rightarrow 1$ quando $\gamma \rightarrow \gamma_{0}$, uniformemente em $\beta$.

Uma vez obtida uma limitação do atrator $\mathcal{A}_{\gamma}$ em $X_{\gamma}^{\beta}$ uniforme em $\gamma-S$ independe de $\gamma$, a partir da desigualdade (1.17), temos que $\|x\|_{X^{\beta}} \leq K_{2}(\gamma) S, \forall x \in \mathcal{A}_{\gamma}$, com $K_{2}(\gamma) \rightarrow 1$ quando $\gamma \rightarrow \gamma_{0}$, uniformemente em $\beta$. Isso significa que se as normas desses espaços são equivalentes uniformemente em $\gamma$, então a união dos atratores $\cup_{\gamma \in \Lambda^{\prime}} \mathcal{A}_{\gamma}$ está contida em uma bola de $X^{\beta}$, onde $\Lambda^{\prime} \subset \Lambda$.

Agora, se o problema (1.15) referente ao operador $A$ estiver posto em um aberto $\Omega \subset \mathbb{R}^{n}$ com a propriedade da extensão $C^{m}, X=L_{p}(\Omega), 1 \leq p<\infty$, e $D(A) \subset W^{m, p}(\Omega)$ para algum $m \geq 1$, o Teorema 1.3 .3 se aplica e,

$$
X^{\beta} \subset C^{v}(\Omega) \text {, quando } 0 \leq v<m \beta-\frac{n}{p} .
$$

Nesse contexto, então, conseguimos uma limitação da família de atratores em $L_{\infty}(\Omega)$. Mais formalmente, 
Teorema 1.4.6 Sejam $\Omega \subset \mathbb{R}^{n}$ um aberto com a propriedade da extensão $C^{m}, X=L_{p}(\Omega), 1 \leq p<\infty e$ $\left\{A_{\gamma}\right\}_{\gamma \in \Lambda}$ uma família de operadores positivos definidos, autoadjuntos e com resolvente compacto em $X$, com $\Lambda$ um espaço topológico e $A=A_{\gamma_{0}}, D(A) \subset W^{m, p}(\Omega)$ para alguma $m \geq 1$.

Suponha que a família $\left\{A_{\gamma}\right\}_{\gamma \in \Lambda}$ é tal que é possível estabelecer um setor e uma constate $M$ comuns a todos os seus elementos e as condições (i)-(iii) do Teorema 1.4.5 estão satisfeitas.

Considere o problema (1.15). Se as suas soluções estiverem globalmente definidas, gerando um semigrupo $T_{\gamma}\left(t, x_{0}\right)$, e este admitir um atrator global $\mathcal{A}_{\gamma}$ que é limitado em $X_{\gamma}^{\alpha}$, para algum $0 \leq \alpha<1$, uniformemente em $\gamma$ e se ainda $f\left(\cup_{\gamma \in \Lambda} \mathcal{A}_{\gamma}\right)$ for também limitada, então a família de atratores é limitada em $L_{\infty}(\Omega)$, uniformemente em $\gamma$, desde que as normas dos espaços $X^{\beta}$ e $X_{\gamma}^{\beta}, \alpha<\beta<1$ e $0<m \beta-\frac{n}{p}$, sejam equivalentes uniformemente em $\gamma$. 


\section{Capítulo 2}

\section{Limitação uniforme dos atratores para uma família de perturbações suaves de um domínio}

Neste capítulo, com base em alguns resultados obtidos em Barbosa, Pereira e Pereira (2016) e em Pereira e Pereira (2007), estudamos uma família de problemas parabólicos semilineares,

$$
\left\{\begin{array}{l}
u_{t}(x, t)=\Delta u(x, t)-a u(x, t)+f(u(x, t)), \quad x \in \Omega_{h}, t>0, \\
\frac{\partial u}{\partial N}(x, t)=g(u(x, t)), \quad x \in \partial \Omega_{h}, t>0,
\end{array}\right.
$$

onde $\Omega$ é um aberto limitado do $\mathbb{R}^{2}$ com fronteira regular de classe $C^{m}, m \geq 2, \Omega_{h}=h(\Omega)$ e $h$ pertence a um conjunto de difeomorfismos suficientemente próximos da identidade na norma $C^{m}$. Mostramos que o problema está bem posto em um espaço conveniente, que o semigrupo associado possui atrator global $\mathcal{A}_{h}$ e, a partir do que foi desenvolvido no primeiro capítulo, que $\left\{\mathcal{A}_{h} \mid\left\|h-i_{\Omega}\right\|_{C^{3}\left(\Omega, \mathbb{R}^{2}\right)}<\epsilon_{0}\right\}$ é uniformemente limitada em $L_{\infty}(\Omega)$, para $\epsilon_{0}$ suficientemente pequeno.

\subsection{Introdução: o problema e a redução a um domínio fixo}

Nesta seção, descrevemos o problema de interesse e o método que utilizamos para estudá-lo. 
Sejam $\Omega$ um aberto limitado do $\mathbb{R}^{2}$ com fronteira regular de classe $C^{m}$ e $a$ um número positivo. Consideremos o problema parabólico semilinear com condições de fronteira de Neumann não lineares:

$$
\left\{\begin{array}{l}
u_{t}(x, t)=\Delta u(x, t)-a u(x, t)+f(u(x, t)), x \in \Omega, t>0, \\
\frac{\partial u}{\partial N}(x, t)=g(u(x, t)), \quad x \in \partial \Omega, t>0 .
\end{array}\right.
$$

Estamos interessados em obter uma limitação uniforme dos atratores em relação às perturbações suaves do domínio $\Omega$. Ocorre que os espaços de funções variam conforme as regiões se alteram. Um modo de superar tal dificuldade é realizar uma "mudança de variável"de forma a trazer o problema de volta para a região fixa. Trata-se da abordagem desenvolvida em Henry (2005) e a qual seguiremos.

Tomemos $h \in \operatorname{Diff}^{m}(\Omega)=\left\{h \in C^{m}\left(\Omega, \mathbb{R}^{2}\right) \mid\right.$ h é injetora e $\frac{1}{\left|\operatorname{det} h^{\prime}(x)\right|}$ é limitado em $\left.\Omega\right\}$. Para h tal que $\left\|h-i_{\Omega}\right\|_{C^{m}\left(\Omega, \mathbb{R}^{2}\right)}$ é pequena, podemos definir o "pull-back"de $h, h^{*}: C^{m}(h(\Omega)) \rightarrow C^{m}(\Omega)$ por $h^{*}(\phi)=\phi \circ h$ que é um isomorfismo com inversa $\left(h^{-1}\right)^{*}$.

Agora, não é difícil ver que $v(\cdot, t)$ satisfaz $(2.1)$ em $\Omega_{h}=h(\Omega)$ se, e somente se, $u(\cdot, t)=h^{*}(v(\cdot, t))$ (isto é, $u(x, t)=v(h(x), t)$ ) verifica:

$$
\left\{\begin{array}{l}
u_{t}(x, t)=h^{*} \Delta_{\Omega_{h}} h^{*-1} u(x, t)-a u(x, t)+f(u(x, t)), \quad x \in \Omega, t>0, \\
h^{*} \frac{\partial}{\partial N_{\Omega_{h}}} h^{*-1} u(x, t)=g(u(x, t)), \quad x \in \partial \Omega, t>0,
\end{array}\right.
$$

onde $h^{*} \Delta_{\Omega_{h}} h^{*-1} u(x, t)=\Delta_{\Omega_{h}}\left(u \circ h^{-1}\right)(h(x))$ e $h^{*} \frac{\partial}{\partial N_{\Omega_{h}}} h^{*-1} u(x)=\frac{\partial}{\partial N_{\Omega_{h}}}\left(u \circ h^{-1}\right)(h(x))$.

\subsection{Setorialidade dos operadores perturbados em vários espaços}

Nesta seção, demonstramos que a família de operadores diferenciais $-h^{*} \Delta_{\Omega_{h}} h^{*-1}+a I, h \in$ $\operatorname{Diff}^{2}(\Omega)$, com h suficientemente próximo de $i_{\Omega}$, é setorial em $L^{2}(\Omega)$ e em $H^{-1}(\Omega)$.

\subsubsection{Setorialidade em $L^{2}$}

Seja $A_{h}=h^{*}\left(B_{h}-a\right) h^{*-1}$ definido na região fixa $\Omega$, onde $B_{h}$ denota o operador Laplaciano com condições de fronteira de Neumann homogêneas em $h(\Omega)$,

$$
B_{h}: D\left(B_{h}\right) \subset L^{2}(h(\Omega)) \rightarrow L^{2}(h(\Omega))
$$

sendo

$$
D\left(B_{h}\right)=H^{2}(h(\Omega))_{N}=\left\{v \in H^{2}(h(\Omega)) \mid \frac{\partial v}{\partial N_{h(\Omega)}}=0\right\}, B_{h}(v)=\Delta_{h(\Omega)} v .
$$


É conhecido que $-A_{h}$ é um operador positivo e autoadjunto em $L^{2}(\Omega)$, considerando-se o produto interno com o peso $\left|J_{h}\right|$, o módulo do determinante Jacobiano de $h$. Denotaremos a norma em $L^{2}(\Omega)$ originada por tal produto interno como $\|\cdot\|_{J}$. Esse espaço é essencialmente o mesmo que $L^{2}(\Omega)$ com a norma usual $\|\cdot\|$, istoé, $K_{2}(h)\|\cdot\| \leq\|\cdot\|_{J} \leq K_{1}(h)\|\cdot\| \operatorname{com} K_{1}(h), K_{2}(h) \rightarrow 1$ quando $h \rightarrow i_{\Omega} . \mathrm{E}$,

$$
D\left(-A_{h}\right)=H^{2}(\Omega)_{N^{*}}=\left\{u \in H^{2}(\Omega) \mid h^{*} \frac{\partial}{\partial N_{h(\Omega)}} h^{*-1} u(x)=\nabla u(x) \cdot\left[D h^{-1}\right]_{h(x)} N_{h(\Omega)}(h(x))=0, \forall x \in \partial \Omega\right\} .
$$

Em geral, não é verdadeira a inclusão $D\left(-A_{i_{\Omega}}\right) \subset D\left(-A_{h}\right)$. O próximo lema, porém, nos mostra que sempre podemos admitir tal hipótese, e mais $D\left(-A_{i_{\Omega}}\right)=D\left(-A_{h}\right)$, desde que tenhamos um pouco mais de regularidade do domínio $\Omega$ e de $h$. Destacamos a importância que esse resultado pode ter em outros contextos, pois permite fixar o domínio de $-A_{h}$ quando $h$ varia.

Lema 2.2.1 Seja $\Omega$ um aberto limitado do $\mathbb{R}^{2}$ com fronteira regular de classe $C^{3}$ e $h \in$ Diff $^{3}(\Omega)$ com $\left\|h-i_{\Omega}\right\|_{C^{3}\left(\Omega, \mathbb{R}^{2}\right)}$ pequeno. Então, para uma tal $h$, existe uma outra transformação $\tilde{h}$ tal que $\tilde{h}(\Omega)=h(\Omega) e$ $\tilde{h}^{*} \frac{\partial}{\partial N_{\tilde{h}(\Omega)}} \tilde{h}^{*-1} u=\frac{\partial u}{\partial N_{\Omega}}$ em $\partial \Omega, \forall u \in H^{2}(\Omega)$.

\section{Demonstração:}

A seguir, construiremos a partir de $h$ uma aplicação $\tilde{h}$ com a propriedade desejada. Para tanto, vamos supor que h está definida em todo o $\mathbb{R}^{2}$ conforme demonstra ser possível o Corolário 1.10 em Henry (2005).

Sejam $N_{\Omega}(p)$ a normal à $p$ em $\partial \Omega$ e $\tilde{N}_{\Omega}(p)=\left[D h^{-1}\right]_{h(p)} N_{\Omega_{h}}(h(p))$, ambas tomadas unitárias. Consideremos $V(\partial \Omega)$ e $\tilde{V}(\partial \Omega)$ vizinhanças de $\partial \Omega$ e,

$$
\begin{aligned}
\varphi: \partial \Omega \times(-\epsilon, \epsilon) & \rightarrow V(\partial \Omega) \\
(p, t) & \mapsto p+t N_{\Omega}(p)
\end{aligned}
$$

e,

$$
\begin{aligned}
\tilde{\varphi}: \partial \Omega \times(-\epsilon, \epsilon) & \rightarrow \tilde{V}(\partial \Omega) \\
(p, t) & \mapsto p+t \tilde{N}_{\Omega}(p) .
\end{aligned}
$$

De acordo com o Teorema 1.5 em Henry (2005), $\varphi$ é um difeomorfismo $C^{2}$ com

$$
\begin{aligned}
\varphi^{-1}: V(\partial \Omega) & \rightarrow \partial \Omega \times(-\epsilon, \epsilon) \\
x & \mapsto(\pi(x), t(x)),
\end{aligned}
$$


onde $\pi(x)=$ o ponto mais próximo a $x$ em $\partial \Omega$ e $t(x)= \pm \operatorname{dist}(x, \pi(x)), \pi \in C^{2}, t \in C^{3}$. Para $h$ próximo de $i_{\Omega}$, como $\varphi$ e $\tilde{\varphi}$ ficam $C^{2}$ próximas, $\tilde{\varphi}$ também é um difeomorfismo $C^{2}$. Vale complementar que no caso de $\Omega \subset \mathbb{R}^{2}$, existe uma transformação conforme $\rho$ que leva $\Omega$ no disco, neste $\varphi^{-1}$ pode ser descrita explicitamente.

Se $\psi_{h}=\tilde{\varphi} \circ \varphi^{-1}$, segue que,

$$
\begin{aligned}
\psi_{h}: V(\partial \Omega) & \rightarrow \tilde{V}(\partial \Omega) \\
x & \mapsto \pi(x)+t(x) \tilde{N}_{\Omega}(\pi(x)) .
\end{aligned}
$$

Observemos que $\psi_{h}$ é um difeomorfismo $C^{2},\left(\psi_{h}\right)_{\mid \partial \Omega}=i_{\partial \Omega}$ e, se $p \in \partial \Omega$,

$$
D \psi_{h}(p) \cdot N_{\Omega}(p)=\lim _{t \rightarrow 0} \frac{\psi_{h}\left(p+t N_{\Omega}(p)\right)-\psi_{h}(p)}{t}=\lim _{t \rightarrow 0} \frac{p+t \tilde{N}_{\Omega}(p)-p}{t}=\tilde{N}_{\Omega}(p) .
$$

Agora, tomemos uma extensão suave de $\psi_{h}, \tilde{\psi}_{h}$, para o interior de $\Omega$. Por exemplo, $\tilde{\psi}_{h}$ pode ser igual à identidade fora de uma vizinhança conveniente da fronteira que continuaremos a denotar por $V(\partial \Omega)$. Do Cálculo, para $\epsilon>0$, existe uma função suave $\theta: \mathbb{R} \rightarrow \mathbb{R}$ tal que $\theta(x)=1, x \in\left[-\frac{\epsilon}{2}, \frac{\epsilon}{2}\right]$ e $\theta(x)=0, x \leq \epsilon$ ou $x \geq \epsilon$. Assim, basta fazermos

$$
\tilde{\psi}(p)=\pi(p)+t(p)\left[\theta(t(p)) \tilde{N}_{\Omega}(\pi(p))+(1-\theta(t(p))) N_{\Omega}(\pi(p))\right]
$$

Definamos então $\tilde{h}=h \circ \tilde{\psi}_{h}: V(\partial \Omega) \cup(\Omega \backslash V(\partial \Omega)) \rightarrow \tilde{V}\left(\partial \Omega_{h}\right) \cup\left(\Omega_{h} \backslash \tilde{V}\left(\partial \Omega_{h}\right)\right)$. Temos que $\tilde{h}^{-1}=\tilde{\psi}_{h}^{-1} \circ h^{-1} \mathrm{e}$, se $x \in \partial \Omega, \tilde{h}(x)=h(x)$. Para $x \in \partial \Omega$,

$$
D \tilde{h}_{\tilde{h}(x)}^{-1}=D\left(\tilde{\psi}_{h}^{-1} \circ h^{-1}\right)_{h(x)}=D \psi_{h}^{-1}\left(h^{-1} h(x)\right) D h^{-1}(h(x))=D \psi_{h}^{-1}(x) D h^{-1}(h(x))
$$

e

$$
D \tilde{h}_{\tilde{h}(x)}^{-1} \cdot N_{\Omega_{\tilde{h}}}(\tilde{h}(x))=D \tilde{h}_{h(x)}^{-1} \cdot N_{\Omega_{h}}(h(x))=D \psi_{h}^{-1}(x) D h^{-1}(h(x)) N_{\Omega_{h}}(h(x))=N_{\Omega}(x),
$$

onde usamos a expressão (2.3), o fato de $\tilde{\psi}_{h}$ ser a identidade na fronteira de $\Omega$ e que $\tilde{h}(\Omega)=$ $h(\Omega)$. Isso significa que se $u \in H^{2}(\Omega)$ satisfaz $\nabla u(x) \cdot N_{\Omega}(x)=0, x \in \partial \Omega$, também vale que $\nabla u(x) \cdot D \tilde{h}_{\tilde{h}(x)}^{-1} \cdot N_{\Omega_{\tilde{h}}}(\tilde{h}(x))=0$ e, portanto, $D\left(-A_{i_{\Omega}}\right) \subset D\left(-A_{\tilde{h}}\right)$. Analogamente, $D\left(-A_{\tilde{h}}\right) \subset D\left(-A_{i_{\Omega}}\right)$.

Por fim, como $\psi_{h}(x)=\pi(x)+t(x)\left(D h^{-1}(h(x)) N_{\Omega_{h}}(h(x))\right)$, para $\left\|h-i_{\Omega}\right\|_{C^{3}\left(\Omega, \mathbb{R}^{2}\right)}$ pequeno, $\psi_{h}$ se aproxima da identidade na norma $C^{2}$ e, resulta que $\tilde{h}$ converge para $i_{\Omega}$ em $C^{2}\left(\Omega, \mathbb{R}^{2}\right)$.

Com base nesse importante resultado, no que segue, admitiremos que $\Omega$ tem fronteira regular de classe $C^{3}$ e $h \in \operatorname{Diff}^{3}(\Omega)$.

Lema 2.2.2 A família de operadores $\left\{-A_{h}\right\}$ satisfaz $\left\|\left(A_{h}-A_{i_{\Omega}}\right) u\right\|_{L^{2}(\Omega)} \leq \varepsilon(h)\left\|A_{i_{\Omega}} u\right\|_{L^{2}(\Omega)}+K(h) \|$ $u \|_{L^{2}(\Omega)} ; \forall u \in D\left(-A_{i_{\Omega}}\right), \operatorname{com} \lim _{h \rightarrow i_{\Omega}} \varepsilon(h)=0 e \lim _{h \rightarrow i_{\Omega}} K(h)=0$. 


\section{Demonstração:}

Escrevamos $h(x)=h\left(x_{1}, x_{2}\right)=\left(h_{1}(x), h_{2}(x)\right)=\left(y_{1}, y_{2}\right)=y, x \in \Omega$. Para $i=1,2$, temos que:

$$
\begin{aligned}
\left(h^{*} \frac{\partial}{\partial y_{i}} h^{*-1}(u)\right)(x) & =\frac{\partial}{\partial y_{i}}\left(u \circ h^{-1}\right)(h(x)) \\
& =\sum_{j=1}^{2} \frac{\partial u}{\partial x_{j}}\left(h^{-1}(y)\right) \frac{\partial h_{j}^{-1}}{\partial y_{i}}(y) \\
& =\sum_{j=1}^{2}\left[\left(\frac{\partial h}{\partial x}\right)^{-1}\right]_{j, i}(x) \frac{\partial u}{\partial x_{j}}(x) \\
& =\sum_{j=1}^{2} b_{i j}(x) \frac{\partial u}{\partial x_{j}}(x)
\end{aligned}
$$

onde $b_{i j}(x)$ é $i, j$ entrada da inversa transposta da matriz Jacobiana $\left[\frac{\partial h_{i}(x)}{\partial x_{j}}\right]_{i, j=1}^{2}$. Daí,

$$
\begin{aligned}
\left(h^{*} \frac{\partial^{2}}{\partial y_{i}^{2}} h^{*-1}(u)\right)(x) & =\sum_{k=1}^{2} b_{i k}(x)\left(\sum_{j=1}^{2} \frac{\partial b_{i j}}{\partial x_{k}}(x) \cdot \frac{\partial u}{\partial x_{j}}(x)+b_{i j}(x) \frac{\partial^{2} u}{\partial x_{k} \partial x_{j}}(x)\right) \\
& =\sum_{j, k=1}^{2} b_{i k}(x) b_{i j}(x) \frac{\partial^{2} u}{\partial x_{k} \partial x_{j}}(x)+\sum_{j, k=1}^{2} b_{i k}(x) \frac{\partial b_{i j}}{\partial x_{k}}(x) \frac{\partial u}{\partial x_{j}}(x) \\
& =\frac{\partial^{2} u}{\partial x_{i}^{2}}(x)+L_{i}(u)(x),
\end{aligned}
$$

sendo $L_{i}(u)(x)=\left(b_{i i}^{2}(x)-1\right) \frac{\partial^{2} u}{\partial x_{i}^{2}}+\sum_{j, k=1}^{2}\left(1-\delta_{i, j, k}\right) b_{i k}(x) b_{i j}(x) \frac{\partial^{2} u}{\partial x_{k} \partial x_{j}}(x)+\sum_{j, k=1}^{2} b_{i k}(x) \frac{\partial b_{i j}}{\partial x_{k}}(x) \frac{\partial u}{\partial x_{j}}(x)$, onde $\delta_{i, j, k}=1$ se $i=j=k$ e 0 caso contrário.

Logo,

$$
h^{*} \Delta_{h(\Omega)} h^{*-1}(u)=\Delta_{\Omega}(u)+L u
$$

com

$$
L u=\sum_{i=1}^{2} L_{i} u .
$$

Como $b_{j k} \rightarrow \delta_{j k}$ em $C^{1}(\bar{\Omega})$ quando $h \rightarrow i_{\Omega}$ em $C^{2}\left(\bar{\Omega}, \mathbb{R}^{2}\right)$, os coeficientes de L vão à zero quando $h \rightarrow i_{\Omega} \operatorname{em} C^{2}\left(\bar{\Omega}, \mathbb{R}^{2}\right)$. Por Agmon, Douglis e Nirenberg (1955),

$$
\|L u\|_{L^{2}(\Omega)} \leq \varepsilon(h)\left\|\Delta_{\Omega} u\right\|_{L^{2}(\Omega)}+K(h)\|u\|_{L^{2}(\Omega)},
$$

com $\varepsilon(h)$ e $K(h)$ convergindo a 0 à medida que $h \rightarrow i_{\Omega}$ em $C^{2}\left(\bar{\Omega}, \mathbb{R}^{2}\right)$, donde segue o desejado.

Aplicando então o Lema 3.1 em Pereira e Pereira (2007), obtemos que, para $h$ suficientemente próximo de $i_{\Omega},-A_{h}$ é setorial e podemos estabelecer um setor e uma constante $M$ comuns a tais operadores. 


\subsubsection{Setorialidade em $H^{-1}$}

Neste ponto, vamos estender o operador $A_{h}$ para um operador $A_{h,-1}: L^{2}(\Omega) \rightarrow H^{-2}(\Omega)$ usando dualidade e, através de interpolação, definimos o operador $A_{h,-1 / 2}: H^{1}(\Omega) \rightarrow H^{-1}(\Omega)$.

Se $u \in D\left(-A_{h}\right), \psi \in H^{1}(\Omega), v=u \circ h^{-1}$ e $J_{h}$ denota o determinante da matriz Jacobiana $\left[\frac{\partial h_{i}(x)}{\partial x_{j}}\right]_{i, j=1}^{2}$, encontramos, integrando por partes,

$$
\begin{aligned}
& <-A_{h} u, \psi>_{-1,1}=-\int_{\Omega}\left(h^{*} B_{h} h^{*-1} u\right)(x) \psi(x) d x+\int_{\Omega} a u(x) \psi(x) d x \\
& =-\int_{\Omega} B_{h}\left(u \circ h^{-1}\right)(h(x)) \psi(x) d x+a \int_{\Omega} u(x) \psi(x) d x \\
& =-\int_{\Omega_{h}} B_{h} v(y) \psi\left(h^{-1}(y)\right) \frac{1}{\left|J_{h}\left(h^{-1}(y)\right)\right|} d y+a \int_{\Omega_{h}} u\left(h^{-1}(y)\right) \psi\left(h^{-1}(y)\right) \frac{1}{\left|J_{h}\left(h^{-1}(y)\right)\right|} d y \\
& =\int_{\Omega_{h}} \nabla_{\Omega_{h}} v(y) \cdot \nabla_{\Omega_{h}} \psi\left(h^{-1}(y)\right) \frac{1}{\left|J_{h}\left(h^{-1}(y)\right)\right|} d y \\
& -\int_{\partial \Omega_{h}} \frac{\partial v}{\partial N_{\Omega_{h}}}(y) \psi\left(h^{-1}(y)\right) \frac{1}{\left|J_{h}\left(h^{-1}(y)\right)\right|} d \sigma(y)+a \int_{\Omega_{h}} u\left(h^{-1}(y)\right) \psi\left(h^{-1}(y)\right) \frac{1}{\left|J_{h}\left(h^{-1}(y)\right)\right|} d y \\
& =\int_{\Omega_{h}} \nabla_{\Omega_{h}} v(y) \cdot \nabla_{\Omega_{h}} \psi\left(h^{-1}(y)\right) \frac{1}{\left|J_{h}\left(h^{-1}(y)\right)\right|} d y \\
& +a \int_{\Omega_{h}} u\left(h^{-1}(y)\right) \psi\left(h^{-1}(y)\right) \frac{1}{\left|J_{h}\left(h^{-1}(y)\right)\right|} d y \\
& =\int_{\Omega} h^{*} \nabla_{\Omega_{h}} h^{*-1} u(x) \cdot h^{*} \nabla_{\Omega_{h}} h^{*-1} \frac{\psi}{\left|J_{h}\right|}(x)\left|J_{h}(x)\right| d x+a \int_{\Omega} u(x) \psi(x) d x .
\end{aligned}
$$

Como (2.4) está bem definida para $u \in H^{1}(\Omega)$, ela nos fornece a expressão de $-A_{h,-1 / 2}$ para qualquer $u$ em seu domínio. A seguir mostraremos que $-A_{h,-1 / 2}$ é setorial.

Lema 2.2.3 A família de operadores $\left\{-A_{h,-1 / 2}\right\}_{h \in D i f f{ }^{2}(\Omega)}$ verifica $\left\|\left(A_{h,-1 / 2}-A_{i_{\Omega},-1 / 2}\right) u\right\|_{H^{-1}(\Omega)} \leq \varepsilon(h) \|$ $A_{i_{\Omega},-1 / 2} u\left\|_{H^{-1}(\Omega)}+K(h)\right\| u \|_{H^{-1}(\Omega)}, \forall u \in D\left(-A_{i_{\Omega},-1 / 2}\right)$, com $\varepsilon(h)$ e $K(h)$ funções positivas tais que $\lim _{h \rightarrow i_{\Omega}} \varepsilon(h)=0$ e $\lim _{h \rightarrow i_{\Omega}} K(h)=0$.

\section{Demonstração:}

De fato, provaremos que,

$$
\left|<\left(A_{h,-1 / 2}-A_{i_{\Omega},-1 / 2}\right) u, \psi>_{-1,1}\right| \leq \varepsilon(h)\|u\|_{H^{1}(\Omega)}\|\psi\|_{H^{1}(\Omega)},
$$


para todo $u \in H^{1}(\Omega)$ e todo $\psi \in H^{1}(\Omega) \operatorname{com} \lim _{h \rightarrow i_{\Omega}} \varepsilon(h)=0$. Vejamos,

$$
\begin{aligned}
\left|<\left(A_{h,-1 / 2}-A_{i_{\Omega},-1 / 2}\right) u, \psi>_{-1,1}\right| & =\left|\int_{\Omega}\left(h^{*} \nabla_{\Omega_{h}} h^{*-1}-\nabla_{\Omega}\right) u \cdot h^{*} \nabla_{\Omega_{h}} h^{*-1}\left(\frac{\psi}{\left|J_{h}\right|}\right)\right| J_{h} \mid d x \\
& +\int_{\Omega} \nabla_{\Omega} u \cdot\left(h^{*} \nabla_{\Omega_{h}} h^{*-1}-\nabla_{\Omega}\right)\left(\frac{\psi}{\left|J_{h}\right|}\right)\left|J_{h}\right| d x \\
& +\int_{\Omega} \nabla_{\Omega} u \cdot \nabla_{\Omega}\left(\frac{\psi}{\left|J_{h}\right|}\right)\left|J_{h}\right| d x-\int_{\Omega} \nabla_{\Omega} u \cdot \nabla_{\Omega} \psi d x \mid \\
& =\left|\int_{\Omega}\left(h^{*} \nabla_{\Omega_{h}} h^{*-1}-\nabla_{\Omega}\right) u \cdot h^{*} \nabla_{\Omega_{h}} h^{*-1}\left(\frac{\psi}{\left|J_{h}\right|}\right)\right| J_{h} \mid d x \\
& +\int_{\Omega} \nabla_{\Omega} u \cdot\left(h^{*} \nabla_{\Omega_{h}} h^{*-1}-\nabla_{\Omega}\right)\left(\frac{\psi}{\left|J_{h}\right|}\right)\left|J_{h}\right| d x \\
& +\int_{\Omega} \psi \nabla_{\Omega} u \cdot \nabla_{\Omega}\left(\frac{1}{\left|J_{h}\right|}\right)\left|J_{h}\right| d x+\int_{\Omega} \nabla_{\Omega} u \cdot \nabla_{\Omega} \psi d x-\int_{\Omega} \nabla_{\Omega} u \cdot \nabla_{\Omega} \psi d x \mid \\
& =\int_{\Omega}\left|\left(h^{*} \nabla_{\Omega_{h}} h^{*-1}-\nabla_{\Omega}\right) u \cdot h^{*} \nabla_{\Omega_{h}} h^{*-1}\left(\frac{\psi}{\left|J_{h}\right|}\right)\right|\left|J_{h}\right| d x \\
& +\int_{\Omega}\left|\nabla_{\Omega} u \cdot\left(h^{*} \nabla_{\Omega_{h}} h^{*-1}-\nabla_{\Omega}\right)\left(\frac{\psi}{\left|J_{h}\right|}\right)\right|\left|J_{h}\right| d x \\
& +\int_{\Omega}\left|\psi \nabla_{\Omega} u \cdot \nabla_{\Omega}\left(\frac{1}{\left|J_{h}\right|}\right)\right|\left|J_{h}\right| d x .
\end{aligned}
$$

Conforme os cálculos feitos no Lema 2.2.2. $\left(h^{*} \frac{\partial}{\partial y_{i}} h^{*-1}(u)\right)(x)=\sum_{j=1}^{2} b_{i j}(x) \frac{\partial u}{\partial x_{j}}(x)$, sendo $b_{i j}(x)$ a $i, j$ entrada da inversa transposta da matriz Jacobiana $\left[\frac{\partial h_{i}(x)}{\partial x_{j}}\right]_{i, j=1}^{2}$. Assim, se $\delta_{i, j}=1$ se $i=j$ e 0 caso contrário,

$$
\left(h^{*} \nabla_{\Omega_{h}} h^{*-1}-\nabla_{\Omega}\right) u(x)=\left[\begin{array}{l}
\sum_{j=1}^{2}\left(b_{1 j}(x)-\delta_{1 j}\right) \frac{\partial u}{\partial x_{j}}(x) \\
\sum_{j=1}^{2}\left(b_{2 j}(x)-\delta_{2 j}\right) \frac{\partial u}{\partial x_{j}}(x)
\end{array}\right],
$$

o que nos permite estimar a primeira integral da desigualdade,

$$
\begin{aligned}
\int_{\Omega} & \left(h^{*} \nabla_{\Omega_{h}} h^{*-1}-\nabla_{\Omega}\right) u \cdot h^{*} \nabla_{\Omega_{h}} h^{*-1}\left(\frac{\psi}{\left|J_{h}\right|}\right)|| J_{h} \mid d x \\
\leq & \max _{x \in \bar{\Omega}}\left\{\left|J_{h}\right|\right\} \int_{\Omega}\left\{\sum_{i=1}^{2}\left[\sum_{j=1}^{2} b_{i j} \frac{\partial}{\partial x_{j}}\left(\frac{\psi}{\left|J_{h}\right|}\right)\right]^{2}\right\}^{\frac{1}{2}}\left\{\sum_{i=1}^{2}\left[\sum_{j=1}^{2}\left(b_{i j}-\delta_{i j}\right) \frac{\partial u}{\partial x_{j}}\right]^{2}\right\}^{\frac{1}{2}} d x \\
\leq & \max _{x \in \bar{\Omega}}\left\{\left|J_{h}\right|\right\}\left\{\int_{\Omega} \sum_{i=1}^{2}\left[\sum_{j=1}^{2} b_{i j} \frac{\partial}{\partial x_{j}}\left(\frac{\psi}{\left|J_{h}\right|}\right)\right]^{2} d x\right\}^{\frac{1}{2}}\left\{\int_{\Omega} \sum_{i=1}^{2}\left[\sum_{j=1}^{2}\left(b_{i j}-\delta_{i j}\right) \frac{\partial u}{\partial x_{j}}\right]^{2} d x\right\}^{\frac{1}{2}} \\
\leq & C_{0}(h)\left(\int_{\Omega} \nabla_{\Omega} \psi \cdot \nabla_{\Omega} \psi d x\right)^{\frac{1}{2}}\left(\int_{\Omega} \nabla_{\Omega} u \cdot \nabla_{\Omega} u d x\right)^{\frac{1}{2}} \\
& +C_{0}^{\prime}(h)\left(\int_{\Omega} \psi^{2} d x\right)^{\frac{1}{2}}\left(\int_{\Omega} \nabla_{\Omega} u \cdot \nabla_{\Omega} u d x\right)^{\frac{1}{2}},
\end{aligned}
$$

onde $C_{0}(h)$ e $C_{0}^{\prime}(h)$ são funções dependendo de $b_{i j}, \frac{\partial}{\partial x_{j}}\left(\frac{1}{\left|J_{h}\right|}\right)$ e que tendem a zero quando $h \rightarrow i_{\Omega}$ em $C^{2}\left(\Omega, \mathbb{R}^{2}\right)$. 
Analogamente, estimamos as outras integrais:

$$
\begin{aligned}
\int_{\Omega} & \left|\nabla_{\Omega} u \cdot\left(h^{*} \nabla_{\Omega_{h}} h^{*-1}-\nabla_{\Omega}\right)\left(\frac{\psi}{\left|J_{h}\right|}\right)\right|\left|J_{h}\right| d x \\
\leq & \max _{x \in \bar{\Omega}}\left\{\left|J_{h}\right|\right\}\left\{\int_{\Omega} \sum_{j=1}^{2}\left[\frac{\partial u}{\partial x_{j}}\right]^{2} d x\right\}^{\frac{1}{2}}\left\{\int_{\Omega} \sum_{i=1}^{2}\left[\sum_{j=1}^{2}\left(b_{i j}-\delta_{i j}\right) \frac{\partial}{\partial x_{j}}\left(\frac{\psi}{\left|J_{h}\right|}\right)\right]^{2} d x\right\}^{\frac{1}{2}} \\
\leq & C_{1}(h)\left(\int_{\Omega} \nabla_{\Omega} \psi \cdot \nabla_{\Omega} \psi d x\right)^{\frac{1}{2}}\left(\int_{\Omega} \nabla_{\Omega} u \cdot \nabla_{\Omega} u d x\right)^{\frac{1}{2}} \\
+ & C_{1}^{\prime}(h)\left(\int_{\Omega} \psi^{2} d x\right)^{\frac{1}{2}}\left(\int_{\Omega} \nabla_{\Omega} u \cdot \nabla_{\Omega} u d x\right)^{\frac{1}{2}}
\end{aligned}
$$

e,

$$
\begin{aligned}
\int_{\Omega} & \left|\psi \nabla_{\Omega} u \cdot \nabla_{\Omega}\left(\frac{1}{\left|J_{h}\right|}\right)\right|\left|J_{h}\right| d x \\
\leq & \max _{x \in \bar{\Omega}}\left\{\left|J_{h}\right|\right\} \int_{\Omega} \psi\left[\sum_{j=1}^{2}\left(\frac{\partial u}{\partial x_{j}}\right)^{2}\right]^{\frac{1}{2}}\left[\sum_{j=1}^{2}\left(\frac{\partial}{\partial x_{j}}\left(\frac{1}{\left|J_{h}\right|}\right)\right)^{2}\right]^{\frac{1}{2}} d x \\
\leq & C_{2}(h)\left(\int_{\Omega} \psi^{2} d x\right)^{\frac{1}{2}}\left(\int_{\Omega}\left|\nabla_{\Omega} u\right|^{2} d x\right)^{\frac{1}{2}}
\end{aligned}
$$

com $C_{1}(h), C_{1}^{\prime}(h)$, e $C_{2}(h) \rightarrow 0$ quando $h \rightarrow i_{\Omega}$ em $C^{2}\left(\Omega, \mathbb{R}^{2}\right)$. E o resultado segue.

A setorialidade então de $-A_{h,-1 / 2}$, bem como a determinação de um setor e uma constante $M$ comuns a tais operadores, segue novamente do Lema 3.1 em Pereira e Pereira (2007), desde que h esteja suficientemente próximo de $i_{\Omega}$ em $C^{2}$.

\subsection{O problema abstrato em uma escala de espaços de Banach}

Nesta seção, nosso objetivo é colocar o problema (2.2) em um espaço abstrato conveniente. Sabemos pela primeira parte do trabalho que os domínios $X_{h}^{\alpha}$ (respectivamente $X_{h,-1 / 2}^{\alpha}$ ), $\alpha \geq 0$, das potências fracionárias de $-A_{h}$ (respectivamente $-A_{h,-1 / 2}$ ) são espaços de Banach; $X_{h}^{0}=L^{2}(\Omega)\left(X_{h,-1 / 2}^{0}=H^{-1}(\Omega)\right), X_{h}^{1}=D\left(-A_{h}\right)\left(X_{h,-1 / 2}^{1}=D\left(-A_{h,-1 / 2}\right)\right), X_{h}^{\alpha}=H^{2 \alpha}(\Omega)$ quando $2 \alpha$ é um número inteiro, $X_{h}^{-\alpha}=\left(X_{h}^{\alpha}\right)^{\prime}$ para $\alpha \geq 0$, onde ' denota o espaço dual. Observemos também que $X_{h}^{\alpha-\frac{1}{2}}=X_{h,-1 / 2}^{\alpha}$ para $\frac{1}{2} \leq \alpha \leq 1$. Por abuso de notação, escreveremos ainda $X_{h}^{\alpha-\frac{1}{2}}$ em vez de $X_{h,-1 / 2}^{\alpha}$ se $0 \leq \alpha \leq \frac{1}{2}$. 
Teorema 2.3.1 Para $\frac{-1}{2} \leq \beta \leq 0$, definimos o operador $\left(-A_{h,-1 / 2}\right)_{\beta}: X_{h}^{\beta+1} \subset X_{h}^{\beta} \rightarrow X_{h^{\prime}}^{\beta}\left(-A_{h,-1 / 2}\right)_{\beta}:=$ $-\left.A_{h,-1 / 2}\right|_{X_{h}^{\beta+1}}$. Então, $\left(-A_{h,-1 / 2}\right)_{\beta}$ é setorial.

\section{Demonstração:}

Seja $\beta=-1 / 2+\delta$ com $0 \leq \delta \leq \frac{1}{2}$. Temos que $\left(-A_{h,-1 / 2}\right)_{\beta}=\left(-A_{h,-1 / 2}\right)^{-\delta}\left(-A_{h,-1 / 2}\right)\left(-A_{h,-1 / 2}\right)^{\delta}$. Agora, $\left(-A_{h,-1 / 2}\right)^{\delta}$ é uma isometria de $X_{h}^{\beta}\left(=X_{h,-1 / 2}^{\beta+\frac{1}{2}}\right)$ em $X_{h}^{\frac{-1}{2}}\left(=X_{h,-1 / 2}^{0}\right)$. Com efeito, se $x \in$ $X_{h,-1 / 2^{\prime}}^{\beta+\frac{1}{2}}$

$$
\left\|\left(-A_{h,-1 / 2}\right)^{\delta} x\right\|_{X_{h,-1 / 2}^{0}}=\left\|\left(-A_{h,-1 / 2}\right)^{\beta+\frac{1}{2}} x\right\|=\|x\|_{X_{h,-1 / 2}^{\beta+\frac{1}{2}}},
$$

donde segue o desejado.

A partir disso, podemos estabelecer (2.2) como um problema abstrato numa escala de espaços de Banach $\left\{X_{h}^{\beta} \mid \frac{-1}{2} \leq \beta \leq 0\right\}$,

$$
\left\{\begin{array}{l}
u_{t}+\left(-A_{h,-1 / 2}\right)_{\beta}=\left(H_{h,-1 / 2}\right)_{\beta} u, t>t_{0} \\
u\left(t_{0}\right)=u_{0} \in X_{h^{\prime}}^{\eta}
\end{array}\right.
$$

onde $\left(H_{h,-1 / 2}\right)_{\beta}=H(\cdot, h)=\left(F_{h,-1 / 2}\right)_{\beta}+\left(G_{h,-1 / 2}\right)_{\beta}: X_{h}^{\eta} \rightarrow X_{h^{\prime}}^{\beta} \operatorname{com} 0 \leq \eta \leq \beta+1$.

$\bullet\left(F_{h,-1 / 2}\right)_{\beta}=F(\cdot, h): X_{h}^{\eta} \rightarrow X_{h}^{\beta}$ é dada por:

$$
<F(u, h), \phi>_{\beta,-\beta}=\int_{\Omega} f(u) \phi d x, \forall \phi \in X_{h}^{-\beta} .
$$

$\bullet\left(G_{h,-1 / 2}\right)_{\beta}=G(\cdot, h): X_{h}^{\eta} \rightarrow X_{h}^{\beta}$ é definida por:

$$
<G(u, h), \phi>_{\beta,-\beta}=\int_{\partial \Omega} g(\gamma(u)) \gamma(\phi)\left|\frac{J \partial \Omega}{J h}\right| d \sigma(x), \forall \phi \in X_{h}^{-\beta},
$$

sendo $\gamma$ a função traço e $J_{\partial \Omega} h$ o determinante da matriz Jacobiana do difeomorfismo $h: \partial \Omega \rightarrow$ $\partial h(\Omega)$.

\subsection{Existência de soluções locais}

Mostramos a seguir, com algumas hipóteses adicionais sobre as funções $f$ e $g$, que o problema abstrato (2.5) possui solução local. 
Para provar que o problema está bem posto localmente, admitiremos as seguintes condições de regularidade e crescimento para $f$ e $g: \mathbb{R} \rightarrow \mathbb{R}$,

(i) $f \in C^{1}(\mathbb{R}, \mathbb{R})$ e existem números reais $\lambda_{1}>0$ e $L_{1}>0$ tais que:

$$
\left|f\left(u_{1}\right)-f\left(u_{2}\right)\right| \leq L_{1}\left(1+\left|u_{1}\right|^{\lambda_{1}}+\left|u_{2}\right|^{\lambda_{1}}\right)\left|u_{1}-u_{2}\right|, \forall u_{1}, u_{2} \in \mathbb{R} .
$$

(ii) $g \in C^{2}(\mathbb{R}, \mathbb{R})$ e existem números reais $\lambda_{2}>0$ e $L_{2}>0$ tais que:

$$
\left|g\left(u_{1}\right)-g\left(u_{2}\right)\right| \leq L_{2}\left(1+\left|u_{1}\right|^{\lambda_{2}}+\left|u_{2}\right|^{\lambda_{2}}\right)\left|u_{1}-u_{2}\right|, \forall u_{1}, u_{2} \in \mathbb{R} .
$$

Lema 2.4.1 Se $\frac{1}{2}-\frac{1}{2\left(\lambda_{1}+1\right)}<\eta<\beta+1$, então $X_{h}^{\eta} \subset L^{2\left(\lambda_{1}+1\right)}(\Omega)$.

\section{Demonstração:}

De fato, pelo Teorema 1.3.3. $X_{h}^{\eta} \subset W^{k, 2}(\Omega)$ quando $k<2 \eta$.

E, por uma das Desigualdades de Nirenberg - Gagliardo, item (a) da Proposição 1.3.1,॥ $u\left\|_{L^{2\left(\lambda_{1}+1\right)}(\Omega)} \leq C\right\| u \|_{W^{k, 2}(\Omega)}$ se $k \geq 1-\frac{1}{\left(\lambda_{1}+1\right)}$.

Logo, reunindo tais considerações, temos que $X_{h}^{\eta} \subset L^{2\left(\lambda_{1}+1\right)}(\Omega)$ desde que $\eta>\frac{1}{2}-\frac{1}{2\left(\lambda_{1}+1\right)}$.

É importante observar que uma das hipóteses do Teorema 1.3.3 é a de que $\Omega$ tem a propriedade da extensão $C^{m}$ e isso é verdadeiro em nosso contexto conforme o Teorema 1.9 em Henry (2005) e até mesmo para regiões cuja fronteira é apenas Lipschitziana como demonstra Stein (1970).

Lema 2.4.2 Suponha que $f$ satisfaz 2.6 e $\eta>\frac{1}{2}-\frac{1}{2\left(\lambda_{1}+1\right)}$. Então, a aplicação $\left(F_{h,-1 / 2}\right)_{\beta}=F: X_{h}^{\eta} \rightarrow X_{h}^{\beta}$ está bem definida e é limitada em conjuntos limitados de $X_{h}^{\eta}$.

\section{Demonstração:}

Sejam $u \in X_{h}^{\eta}$ e $\phi \in X_{h}^{-\beta}$

$$
\begin{aligned}
& \left|<F(u, h), \phi>_{\beta,-\beta}\right| \leq \int_{\Omega}|f(u)-f(0)||\phi| d x+\int_{\Omega}|f(0)||\phi| d x \\
& \leq L_{1} \int_{\Omega}|u||\phi| d x+L_{1} \int_{\Omega}|u|^{\lambda_{1}+1}|\phi| d x+\int_{\Omega}|f(0)||\phi| d x \\
& \leq L_{1}\|u\|_{L^{2}(\Omega)}\|\phi\|_{L^{2}(\Omega)}+L_{1}\left\|u^{\lambda_{1}+1}\right\|_{L^{2}(\Omega)}\|\phi\|_{L^{2}(\Omega)}+\|f(0)\|_{L^{2}(\Omega)}\|\phi\|_{L^{2}(\Omega)} \\
& \leq L_{1}\|u\|_{L^{2}(\Omega)}\|\phi\|_{L^{2}(\Omega)}+L_{1}\|u\|_{L^{2\left(\lambda_{1}+1\right)}(\Omega)}^{\lambda_{1}+1}\|\phi\|_{L^{2}(\Omega)}+\|f(0)\|_{L^{2}(\Omega)}\|\phi\|_{L^{2}(\Omega)} \\
& \leq L_{1} K_{1} K_{2}\|u\|_{X_{h}^{\eta}}\|\phi\|_{X_{h}^{-\beta}}+L_{1} K_{1} K_{3}^{\lambda_{1}+1}\|u\|_{X_{h}^{\eta}}^{\lambda_{1}+1}\|\phi\|_{X_{h}^{-\beta}}+K_{1}\|f(0)\|_{L^{2}(\Omega)}\|\phi\|_{X_{h}^{-\beta}} \text {, }
\end{aligned}
$$

onde $K_{1}, K_{2}$ e $K_{3}$ são as constantes de imersão de, respectivamente, $X_{h}^{-\beta} \subset L^{2}(\Omega), X_{h}^{\eta} \subset L^{2}(\Omega)$ e $X_{h}^{\eta} \subset$ $L^{2\left(\lambda_{1}+1\right)}(\Omega)$. As duas primeiras são imediatas uma vez que $0 \leq-\beta \leq \frac{1}{2}$ e $0 \leq \eta \leq \beta+1$ e a terceira, 
demonstramos no Lema 2.4.1.

Portanto, se $u \in X_{h}^{\eta}$ e $\phi \in X_{h}^{-\beta}$,

$$
\|F(u, h)\|_{X_{h}^{\beta}} \leq L_{1} K_{1} K_{2}\|u\|_{X_{h}^{\eta}}+L_{1} K_{1} K_{3}^{\lambda_{1}+1}\|u\|_{X_{h}^{\eta}}^{\lambda_{1}+1}+K_{1}\|f(0)\|_{L^{2}(\Omega)}
$$

o que nos mostra que $F$ é limitada em conjuntos limitados de $X_{h}^{\eta}$.

Lema 2.4.3 Suponha que estejam satisfeitas as condições do Lema 2.4.2 e sejam p e q conjugados com $\frac{1}{\lambda_{1}}<p<\infty$. Então, se $\eta>\max \left\{\frac{1}{2}-\frac{1}{2 p \lambda_{1}}, \frac{1}{2}-\frac{1}{2 q}\right\}$, a aplicação F é localmente Lipschitz contínua em u.

\section{Demonstração:}

Sejam $u_{1}, u_{2} \in X_{h}^{\eta}$ e $\phi \in X_{h}^{-\beta}$

$$
\begin{aligned}
\left|<F\left(u_{1}, h\right)-F\left(u_{2}, h\right), \phi>_{\beta,-\beta}\right| & \leq \int_{\Omega} L_{1}\left(1+\left|u_{1}\right|^{\lambda_{1}}+\left|u_{2}\right|^{\lambda_{1}}\right)\left|u_{1}-u_{2}\right||\phi| d x \\
& \leq L_{1}\left(\int_{\Omega}\left(1+\left|u_{1}\right|^{\lambda_{1}}+\left|u_{2}\right|^{\lambda_{1}}\right)^{2}\left|u_{1}-u_{2}\right|^{2} d x\right)^{\frac{1}{2}}\|\phi\|_{L^{2}(\Omega)} \\
& \leq L_{1}\left(\int_{\Omega}\left(1+\left|u_{1}\right|^{\lambda_{1}}+\left|u_{2}\right|^{\lambda_{1}}\right)^{2 p} d x\right)^{\frac{1}{2 p}}\left(\int_{\Omega}\left|u_{1}-u_{2}\right|^{2 q} d x\right)^{\frac{1}{2 q}}\|\phi\|_{L^{2}(\Omega)} \\
& \leq L_{1}\left(|\Omega|^{\frac{1}{2 p}}+\left\|u_{1}^{\lambda_{1}}\right\|_{L^{2 p}(\Omega)}+\left\|u_{2}^{\lambda_{1}}\right\|_{L^{2 p}(\Omega)}\right)\left\|u_{1}-u_{2}\right\|_{L^{2 q}(\Omega)}\|\phi\|_{L^{2}(\Omega)} \\
& \leq L_{1}\left(|\Omega|^{\frac{1}{2 p}}+\left\|u_{1}\right\|_{L^{2 p \lambda_{1}(\Omega)}}^{\lambda_{1}}+\left\|u_{2}\right\|_{L^{2 p \lambda_{1}(\Omega)}}^{\lambda_{1}}\right)\left\|u_{1}-u_{2}\right\|_{L^{2 q}(\Omega)}\|\phi\|_{L^{2}(\Omega)} \\
& \leq L_{1}\left(|\Omega|^{\frac{1}{2 p}}+K_{4}^{\lambda_{1}}\left\|u_{1}\right\|_{X_{h}^{\eta}}^{\lambda_{1}}+K_{4}^{\lambda_{1}}\left\|u_{2}\right\|_{X_{h}^{\eta}}^{\lambda_{1}}\right) K_{5}\left\|u_{1}-u_{2}\right\|_{X_{h}^{\eta}} K_{1}\|\phi\|_{X_{h}^{-\beta},}
\end{aligned}
$$

onde $K_{1}$ é a constante de imersão referente a $X_{h}^{-\beta} \subset L^{2}(\Omega)$ e $K_{4}$ e $K_{5}$ são obtidas pela inclusão de $X_{h}^{\eta} \subset L^{2 p \lambda_{1}}(\Omega)$ e $X_{h}^{\eta} \subset L^{2 q}(\Omega)$ respectivamente, usando o mesmo raciocínio que no Lema 2.4.1 dado que $\eta>\max \left\{\frac{1}{2}-\frac{1}{2 p \lambda_{1}}, \frac{1}{2}-\frac{1}{2 q}\right\}$ e $p>\frac{1}{\lambda_{1}}$.

Logo, se $u_{1}$ e $u_{2}$ pertencem a um subconjunto limitado $U$ de $X_{h}^{\eta}\|u\|_{X_{h}^{\eta}} \leq L, \forall u \in U$,

$$
\left\|F\left(u_{1}, h\right)-F\left(u_{2}, h\right)\right\|_{X_{h}^{\beta}} \leq L_{1}\left(|\Omega|^{\frac{1}{2 p}}+2 K_{4}^{\lambda_{1}} L^{\lambda_{1}}\right) K_{5} K_{1}\left\|u_{1}-u_{2}\right\|_{X_{h}^{\eta}}
$$

donde $F$ é localmente Lipschitziana em $u$.

Para as propriedades de regularidade de $\left(G_{h,-1 / 2}\right)_{\beta}=G$, precisaremos da seguinte função:

$$
\theta(x, h)=\left|\frac{J_{\partial \Omega} h}{J_{h}}(x)\right|, x \in \mathbb{R}^{2} .
$$


Provaremos que $\|\theta\|_{\infty}=\sup \left\{\mid \theta(x, h)\|x \in \partial \Omega\| h-,i_{\Omega} \|_{C^{2}\left(\Omega, \mathbb{R}^{2}\right)}<\epsilon_{0}\right\}$ é finita, para algum $\epsilon_{0}>0$ suficientemente pequeno fixado.

Notemos que para cada $h=h_{0}$ fixo, $\sup _{x \in \partial \Omega} \theta\left(x, h_{0}\right)$ é finito afinal, trata-se de uma função contínua em um compacto. E, devido à convergência de $h$ para $i_{\Omega}$ na norma $C^{2}\left(\Omega, \mathbb{R}^{2}\right)$, o denominador na expressão de $\theta, J_{h}$, converge para 1 uniformemente. Devemos então estudar $J_{\partial \Omega} h$.

Seguindo primeiramente uma abordagem intrínseca, chamemos $M=\partial \Omega$ e $N=h(\partial \Omega)$, ambos de dimensão $n-1$ (em nosso caso, de dimensão 1). Se $f$ é uma função em $N$ a $\mathbb{R}$,

$$
\int_{N} f(y) d \omega_{N}=\int_{M} f(h(x)) J_{\partial \Omega} h d \omega_{M}
$$

sendo $d \omega_{\{\}\}}$o elemento de volume em $M$ ou em $N$ e $J_{\partial \Omega} h$ representa a correção do fator de volume. Por outro lado,

$$
\int_{N} f(y) d \omega_{N}=\int_{M} f(h(x)) h^{*} d \omega_{N}
$$

Assim, se $p \in M$,

$$
\left(h^{*} d \omega_{N}\right)(p)=J_{\partial \Omega} h(p) d \omega_{M}(p) .
$$

Seja $\left\{e_{1}, \cdots, e_{n-1}\right\}$ uma base ortonormal de TpM, o espaço tangente a $p$ em $M$. Usando (2.8),

$$
\left(h^{*} d \omega_{N}\right)(p)\left(e_{1}, \cdots, e_{n-1}\right)=J_{\partial \Omega} h(p) d \omega_{M}(p)\left(e_{1}, \cdots, e_{n-1}\right)=J_{\partial \Omega} h(p) .
$$

Agora,

$$
\left(h^{*} d \omega_{N}\right)(p)\left(e_{1}, \cdots, e_{n-1}\right)=d \omega_{N}(h(p))\left(h_{*} e_{1}, \cdots, h_{*} e_{n-1}\right)=\operatorname{det}\left[\begin{array}{c}
D h_{p} e_{1} \\
\vdots \\
D h_{p} e_{n-1} \\
N_{q}
\end{array}\right],
$$

sendo $q=h(p)$ e $N_{q}$ o vetor normal a $q$ em $N$. Observemos que $N_{q}=\frac{\left(h_{*}^{-1}\right)^{t} N_{p}}{\left\|\left(h_{*}^{-1}\right)^{t} N_{p}\right\|}$, pois se $\left\{f_{1}, \cdots, f_{n-1}\right\}$ é uma base ortonormal de $T q N$, o espaço tangente a $q$ em $N$,

$$
<\left(h_{*}^{-1}\right)^{t} N_{p}, f_{i}>=<N_{p}, h_{*}^{-1} f_{i}>=0, \quad \forall i=1, \cdots, n-1,
$$

uma vez que $h$ leva um espaço tangente no outro. Daí,

$$
J_{\partial \Omega} h(p)=\operatorname{det}\left[\begin{array}{c}
D h_{p} e_{1} \\
\vdots \\
D h_{p} e_{n-1} \\
\frac{\left(h_{*}^{-1}\right)^{t} N_{p}}{\left\|\left(h_{*}^{-1}\right)^{t} N_{p}\right\|}
\end{array}\right]
$$

o que evidencia que $J_{\partial \Omega} h$ é limitado uniformemente em $h$ se $h \rightarrow i_{\Omega}$ em $C^{2}\left(\Omega, \mathbb{R}^{n}\right)$.

Trabalhando em coordenadas, sejam $M, N, p$, e $q$ como acima, $\phi$ e $\psi$ um sistema de vizinhanças 
locais em $p$ e $q$ respectivamente com $\phi(x)=p$ e $\psi(y)=q$. Se $\eta=\psi^{-1} \circ h \circ \phi$,

$$
\left(\psi^{-1} \circ h \circ \phi\right)^{*}(y) d y=\left(J_{\eta}\right)(x) d x .
$$

E, vale também que,

$$
\left(\psi^{-1} \circ h \circ \phi\right)^{*}(y) d y=\left(\phi^{*} \circ h^{*} \circ \psi^{-1 *}\right)(y) d y=J_{\phi} J_{\partial \Omega} h J_{\psi^{-1}} d x
$$

Como $\left\|h-i_{\Omega}\right\|_{C^{2}\left(\Omega, \mathbb{R}^{2}\right)} \rightarrow 0$, a grosso modo, $\phi$ e $\psi$ ficam arbitrariamente próximas e, $J_{\phi}$ e $J_{\psi}$, também, donde $J_{\partial \Omega} h \simeq J_{\eta}$ em $C^{2}$ uniformemente. Lembremos que a fronteira é compacta, sendo necessário finitas vizinhanças coordenadas para recobrí-la.

Lema 2.4.4 Suponha que $g$ satisfaz $2.7, \eta\rangle>\frac{1}{2}-\frac{1}{4\left(\lambda_{2}+1\right)} e \beta<-\frac{1}{4}$. Então a aplicação $\left(G_{h,-1 / 2}\right)_{\beta}=G$ : $X_{h}^{\eta} \rightarrow X_{h}^{\beta}$ está bem definida e é limitada em conjuntos limitados de $X_{h}^{\eta}$ para $\left\|h-i_{\Omega}\right\|_{C^{2}\left(\Omega, \mathbb{R}^{2}\right)}<\epsilon_{0}$.

\section{Demonstração:}

Sejam $u \in X_{h}^{\eta}$ e $\phi \in X_{h}^{-\beta}$

$$
\begin{aligned}
& \left|<G(u, h), \phi>_{\beta,-\beta}\right| \leq \int_{\partial \Omega}|g(\gamma(u))||\gamma(\phi)|\left|\frac{J_{\partial \Omega} h}{J h}\right| d \sigma(x) \\
& \leq\|\theta\|_{\infty}\left(\int_{\partial \Omega}|g(\gamma(u))-g(\gamma(0))||\gamma(\phi)| d \sigma(x)+\int_{\partial \Omega}|g(\gamma(0))||\gamma(\phi)| d \sigma(x)\right) \\
& \leq\|\theta\|_{\infty} L_{2} \int_{\partial \Omega}|\gamma(u)||\gamma(\phi)| d \sigma(x)+\|\theta\|_{\infty} L_{2} \int_{\partial \Omega}|\gamma(u)|^{\lambda_{2}+1}|\gamma(\phi)| d \sigma(x) \\
& +\|\theta\|_{\infty} \int_{\partial \Omega}|g(\gamma(0))||\gamma(\phi)| d \sigma(x) \\
& \leq\|\theta\|_{\infty} L_{2}\|\gamma(u)\|_{L^{2}(\partial \Omega)}\|\gamma(\phi)\|_{L^{2}(\partial \Omega)}+\|\theta\|_{\infty} L_{2}\left\|\gamma(u)^{\lambda_{2}+1}\right\|_{L^{2}(\partial \Omega)}\|\gamma(\phi)\|_{L^{2}(\partial \Omega)} \\
& +\|\theta\|_{\infty}\|g(\gamma(0))\|_{L^{2}(\partial \Omega)}\|\gamma(\phi)\|_{L^{2}(\partial \Omega)} \\
& \leq\|\theta\|_{\infty} L_{2}\left(\|\gamma(u)\|_{L^{2}(\partial \Omega)}+\|\gamma(u)\|_{L^{2\left(\lambda_{2}+1\right)}(\partial \Omega)}^{\lambda_{2}+1}\right)\|\gamma(\phi)\|_{L^{2}(\partial \Omega)} \\
& +\|\theta\|_{\infty}\|g(\gamma(0))\|_{L^{2}(\partial \Omega)}\|\gamma(\phi)\|_{L^{2}(\partial \Omega)} \\
& \leq L_{2}\|\theta\|_{\infty} \overline{K_{3}} \overline{K_{1}}\|u\|_{X_{h}^{\eta}}\|\phi\|_{X_{h}^{-\beta}}+L_{2}\|\theta\|_{\infty}{\overline{K_{2}}}^{\lambda_{2}+1} \overline{K_{1}}\|u\|_{X_{h}^{\eta}}\|\phi\|_{X_{h}^{-\beta}} \\
& +\|\theta\|_{\infty} \overline{K_{1}}\|g(\gamma(0))\|_{L^{2}(\partial \Omega)}\|\phi\|_{X_{h}^{-\beta}}
\end{aligned}
$$

onde as constantes $\overline{K_{1}}, \overline{K_{2}}, \overline{K_{3}}$ referem-se ao seguinte: se $s=2 \eta$, então $X_{h}^{\eta} \subset H^{s}(\Omega)$ e $\gamma$ : $H^{s}(\Omega) \rightarrow L^{\frac{1}{1-s}}(\partial \Omega)$ [Veja Necas (2012)], obtendo $\|\gamma(\phi)\|_{L^{2}(\partial \Omega)} \leq \overline{K_{1}}\|\phi\|_{X_{h}^{-\beta}}$ desde que $-\beta>\frac{1}{4}$, $\|\gamma(u)\|_{L^{2\left(\lambda_{2}+1\right)}(\partial \Omega)} \leq \overline{K_{2}}\|u\|_{X_{h}^{\eta}}$ para $\eta>\frac{1}{2}-\frac{1}{4\left(\lambda_{2}+1\right)}$ e $\|\gamma(u)\|_{L^{2}(\partial \Omega)} \leq \overline{K_{3}}\|u\|_{X_{h}^{\eta}} \operatorname{com} \eta>\frac{1}{4}$. E o resultado segue. 
Lema 2.4.5 Suponha que estejam satisfeitas as condições do Lema 2.4.4 e sejam p e q expoentes conjugados $\operatorname{com} \frac{1}{2 \lambda_{2}}<p<\infty$. Então, se $\eta>\max \left\{\frac{1}{2}-\frac{1}{4 p \lambda_{2}}, \frac{1}{2}-\frac{1}{4 q}\right\}$ e $\beta<-\frac{1}{4}$, a aplicação $G: X_{h}^{\eta} \rightarrow X_{h}^{\beta}$ é uniformemente contínua em $h$ para u em conjuntos limitados de $X_{h}^{\eta}$ e localmente Lipschitz contínua em $u$ para $\left\|h-i_{\Omega}\right\|_{C^{2}\left(\Omega, \mathbb{R}^{2}\right)}<\epsilon_{0}$.

\section{Demonstração:}

$$
\begin{aligned}
& \text { Sejam } u_{1}, u_{2} \in X_{h}^{\eta} \mathrm{e} \phi \in X_{h}^{-\beta}, \\
& \left|<G\left(u_{1}, h\right)-G\left(u_{2}, h\right), \phi>_{\beta,-\beta}\right| \\
& \leq \quad \int_{\partial \Omega}\left|g\left(\gamma\left(u_{1}\right)\right)-g\left(\gamma\left(u_{2}\right)\right)\right||\gamma(\phi)|\left|\frac{\partial_{\partial \Omega} h}{J h}\right| d \sigma(x) \\
& \leq\|\theta\|_{\infty} L_{2} \int_{\partial \Omega}\left(1+\left|\gamma\left(u_{1}\right)\right|^{\lambda_{2}}+\left|\gamma\left(u_{2}\right)\right|^{\lambda_{2}}\right)\left|\gamma\left(u_{1}\right)-\gamma\left(u_{2}\right)\right||\gamma(\phi)| d \sigma(x) \\
& \leq\|\theta\|_{\infty} L_{2}\left(\int_{\partial \Omega}\left(1+\left|\gamma\left(u_{1}\right)\right|^{\lambda_{2}}+\left|\gamma\left(u_{2}\right)\right|^{\lambda_{2}}\right)^{2}\left|\gamma\left(u_{1}\right)-\gamma\left(u_{2}\right)\right|^{2} d \sigma(x)\right)^{\frac{1}{2}}\|\gamma(\phi)\|_{L^{2}(\partial \Omega)} \\
& \leq\|\theta\|_{\infty} L_{2}\|\gamma(\phi)\|_{L^{2}(\partial \Omega)}\left(\int_{\partial \Omega}\left(1+\left|\gamma\left(u_{1}\right)\right|^{\lambda_{2}}+\left|\gamma\left(u_{2}\right)\right|^{\lambda_{2}}\right)^{2 p} d \sigma(x)\right)^{\frac{1}{2 p}}\left(\int_{\partial \Omega}\left|\gamma\left(u_{1}\right)-\gamma\left(u_{2}\right)\right|^{2 q} d \sigma(x)\right)^{\frac{1}{2 q}} \\
& \leq\|\theta\|_{\infty} L_{2}\|\gamma(\phi)\|_{L^{2}(\partial \Omega)}\left\|\gamma\left(u_{1}\right)-\gamma\left(u_{2}\right)\right\|_{L^{2 q}(\partial \Omega)}\left(|\partial \Omega|^{\frac{1}{2 p}}+\left\|\gamma\left(u_{1}\right)\right\|_{L^{2 p \lambda_{2}}(\partial \Omega)}^{\lambda_{2}}+\left\|\gamma\left(u_{2}\right)\right\|_{L^{2 p \lambda_{2}}(\partial \Omega)}^{\lambda_{2}}\right) \\
& \leq\|\theta\|_{\infty} L_{2} \overline{K_{1}}\|\phi\|_{X_{h}^{-\beta}} \overline{K_{5}}\left\|u_{1}-u_{2}\right\|_{X_{h}^{\eta}}\left(|\partial \Omega|^{\frac{1}{2 p}}+\bar{K}_{4}^{\lambda_{2}}\left\|u_{1}\right\|_{X_{h}^{\eta}}^{\lambda_{2}}+\bar{K}_{4}^{\lambda_{2}}\left\|u_{2}\right\|_{X_{h}^{\eta}}^{\lambda_{2}}\right),
\end{aligned}
$$

onde usamos um raciocínio semelhante ao do Lema 2.4.4 anterior para estabelecer as desigualdades: $\|\gamma(\phi)\|_{L^{2}(\partial \Omega)} \leq \overline{K_{1}}\|\phi\|_{X_{h}^{-\beta}}$ se $-\beta>\frac{1}{4},\|\gamma(u)\|_{L^{2 p \lambda_{2}(\partial \Omega)}} \leq \overline{K_{4}}\|u\|_{X_{h}^{\eta}}$ se $\eta>\frac{1}{2}-\frac{1}{4 p \lambda_{2}}$ e $\|\gamma(u)\|_{L^{2 q}(\partial \Omega)} \leq \overline{K_{5}}\|u\|_{X_{h}^{\eta}}$ desde que $\eta>\frac{1}{2}-\frac{1}{4 q}$.

Dessa forma, se $u_{1}, u_{2}$ estão em um conjunto limitado $U$ de $X_{h^{\prime}}^{\eta}\|u\|_{X_{h}^{\eta}} \leq L, \forall u \in U$, temos

$$
\left\|G\left(u_{1}, h\right)-G\left(u_{2}, h\right)\right\|_{X_{h}^{\beta}} \leq\|\theta\|_{\infty} L_{2} \overline{K_{1}}\left(|\partial \Omega|^{\frac{1}{2 p}}+2{\overline{K_{4}}}^{\lambda_{2}} L^{\lambda_{2}}\right) \overline{K_{5}}\left\|u_{1}-u_{2}\right\|_{X_{h}^{\eta}}
$$

com $\|\theta\|_{\infty}$ finito independente de $h$ para $\left\|h-i_{\Omega}\right\|_{C^{2}\left(\Omega, \mathbb{R}^{2}\right)}<\epsilon_{0}$. E segue que $G$ é localmente Lipschitz em $u$.

Agora, se $u \in X_{h^{\prime}}^{\eta} \phi \in X_{h}^{-\beta}$ e $h_{1}, h_{2}$ estão $\delta$ próximas de $i_{\Omega}, \delta<\epsilon_{0}$,

$$
\left|<G\left(u, h_{1}\right)-G\left(u, h_{2}\right), \phi>_{\beta,-\beta}\right| \leq\left\|\theta_{1}-\theta_{2}\right\|_{\infty} \int_{\partial \Omega}|g(\gamma(u))||\gamma(\phi)| d \sigma(x),
$$

com $\left\|\theta_{1}-\theta_{2}\right\|_{\infty}=\sup _{x}\left|\theta\left(x, h_{1}\right)-\theta\left(x, h_{2}\right)\right| \rightarrow 0$ quando $\left\|h_{1}-h_{2}\right\|_{C^{2}\left(\Omega, \mathbb{R}^{2}\right)} \rightarrow 0$. Então, desde que $u$ esteja em um conjunto limitado de $X_{h^{\prime}}^{\eta}$ com um raciocínio análogo ao que fizemos no Lema 2.4.4. temos $\left\|G\left(u, h_{1}\right)-G\left(u, h_{2}\right)\right\|_{X_{h}^{\beta}}<\left\|\theta_{1}-\theta_{2}\right\|_{\infty} C$, onde $C>0$ é uma constante. Daí, basta tomar $\delta$ adequado tal que $\left\|\theta_{1}-\theta_{2}\right\|_{\infty}<\frac{\epsilon}{C}$ e, vemos que $G$ é uniformemente contínua em $h$ para $u$ em conjuntos limitados de $X_{h}^{\eta}$ e $h$ suficientemente próximo de $i_{\Omega}$ em $C^{2}$. 
Teorema 2.4.6 Suponha que $f$ e $g$ satisfazem (2.6) e 2.7) respectivamente, sejam $\beta$ e $\eta$ como nos Lemas 2.4.2, 2.4.3, 2.4.4, 2.4.5, $\eta<\beta+1 e\left\|h-i_{\Omega}\right\|_{C^{2}\left(\Omega, \mathbb{R}^{2}\right)}<\epsilon_{0}$. Então, para qualquer $\left(t_{0}, u_{0}\right) \in \mathbb{R} \times X_{h^{\prime}}^{\eta} o$ problema (2.5) possui uma única solução $u\left(t, t_{0}, u_{0}, h\right)$ com valor inicial $u\left(t_{0}\right)=u_{0}$.

\section{Demonstração:}

Já sabemos que $\left(-A_{h,-1 / 2}\right)_{\beta}$ é um operador setorial em $X_{h}^{\beta}$ com domínio $X_{h}^{\beta+1}$. De acordo com os lemas citados, $\left(H_{h,-1 / 2}\right)_{\beta}$ está bem definida, é localmente Lipschitz contínua e limitada em limitados. Portanto, a afirmação segue do Teorema 1.4.2.

\subsection{Funcional de Lyapunov e existência de solução global}

Nesta seção, demonstramos que as soluções dadas pelo Teorema 2.4.6 estão definidas globalmente sob determinadas hipóteses em $f$ e em $g$.

Suponhamos que existem constantes $c_{0}, d_{0}, d_{0}^{\prime}$, com $d_{0}>d_{0}^{\prime}$, tais que,

$$
\lim _{|u| \rightarrow \infty} \sup \frac{f(u)}{u} \leq c_{0}
$$

e,

$$
\lim _{|u| \rightarrow \infty} \sup \frac{g(u)}{u} \leq d_{0}^{\prime} .
$$

E o primeiro autovalor $\mu_{1}$ do problema

$$
\left\{\begin{array}{l}
-\Delta u+\left(a-c_{0}\right) u=\mu u \text { em } \Omega \\
\frac{\partial u}{\partial N_{\Omega}}=d_{0} u \text { em } \partial \Omega
\end{array}\right.
$$

é positivo.

Observemos que tais hipóteses ainda são válidas para o operador perturbado $h^{*} \Delta_{\Omega_{h}} h^{*-1}$ com condição de fronteira perturbada $h^{*} \frac{\partial u}{\partial N_{\Omega_{h}}} h^{*-1}$ afinal, vimos no Lema 2.2.3 que,

$\left\|\left(A_{h,-1 / 2}-A_{i_{\Omega},-1 / 2}\right) u\right\|_{H^{-1}(\Omega)} \leq \varepsilon(h)\left\|A_{i_{\Omega},-1 / 2} u\right\|_{H^{-1}(\Omega)}+K(h)\|u\|_{H^{-1}(\Omega)}, \forall u \in D\left(-A_{i_{\Omega},-1 / 2}\right)$, com $\varepsilon(h)$ e $K(h)$ funções positivas tais que $\lim _{h \rightarrow i_{\Omega}} \varepsilon(h)=0$ e $\lim _{h \rightarrow i_{\Omega}} K(h)=0$ e os autovalores variam continuamente com $h$. 
Para provarmos existência global, trabalharemos no "espaço de energia"natural $H^{1}(\Omega)\left(\eta=\frac{1}{2}\right.$ e $\left.\beta<\frac{-1}{4}\right)$. Inicialmente, porém, é conveniente trabalharmos em $H^{1}\left(\Omega_{h}\right)$ e considerarmos o problema no domínio perturbado $\Omega_{h}$ em $Y_{h^{\prime}}^{\beta}$, sendo $Y_{h}^{\alpha}$, com $-\frac{1}{2} \leq \alpha \leq 1$, o espaço de potência fracionária para o operador $-\Delta_{\Omega_{h}}+a I$,

$$
\left\{\begin{array}{l}
v_{t}+A_{\beta} v=H_{\beta} v, t>t_{0} \\
v\left(t_{0}\right)=v_{0} \in H^{1}\left(\Omega_{h}\right)
\end{array}\right.
$$

onde $H_{\beta}=F_{\beta}+G_{\beta}: H^{1}\left(\Omega_{h}\right) \rightarrow Y_{h^{\prime}}^{\beta}$

- $F_{\beta}: H^{1}\left(\Omega_{h}\right) \rightarrow Y_{h}^{\beta}$ é dada por:

$$
<F(v), \psi>_{\beta,-\beta}=\int_{\Omega_{h}} f(v) \psi d y, \forall \psi \in Y_{h}^{-\beta} .
$$

- $G_{\beta}: H^{1}\left(\Omega_{h}\right) \rightarrow Y_{h}^{\beta}$ é definida por:

$$
<G(v), \psi>_{\beta,-\beta}=\int_{\partial \Omega_{h}} g(\gamma(v)) \gamma(\psi)\left|\frac{J \partial \Omega}{J h}\right| d \sigma(y), \forall \psi \in Y_{h}^{-\beta} .
$$

Sabe-se que $v$ é uma solução deste problema (2.12) se, e somente se, $u=v \circ h$ satisfaz (2.5) com $\eta=\frac{1}{2}$ de modo que a existência de solução local para (2.12) segue do Teorema 2.4.6.

Lema 2.5.1 Suponha que valem as hipóteses do Teorema 2.4.6 e $f$ e $g$ satisfazem as condições dissipativas 2.9) e 2.10) respectivamente. Seja $W_{h}: H^{1}\left(\Omega_{h}\right) \rightarrow \mathbb{R}$ definida por

$$
W_{h}(v)=\frac{1}{2} \int_{\Omega_{h}}|\nabla v|^{2} d x+\frac{a}{2} \int_{\Omega_{h}}|v|^{2} d x-\int_{\Omega_{h}} F(v) d x-\int_{\partial \Omega_{h}} G(\gamma(v)) d S,
$$

onde a é um número positivo, $F$ e $G: \mathbb{R} \rightarrow \mathbb{R}$ são as primitivas de $f$ e $g$ respectivamente. Então, para $h$ suficientemente próxima de $i_{\Omega}, W_{h}$ é um funcional de Lyapunov para 2.12 e existem constantes $K_{1}$ e $K_{2}$ tais que

$$
W_{h}(v) \leq K_{1}\|v\|_{H^{1}\left(\Omega_{h}\right)}^{2}+K_{2}, \forall v \in H^{1}\left(\Omega_{h}\right)
$$

\section{Demonstração:}

Pela expressão de $W_{h}$, vemos que trata-se de uma função contínua e, se $v$ é uma solução de 2.12 em $H^{1}\left(\Omega_{h}\right)$,

$$
\begin{aligned}
\frac{d}{d t} W_{h}(v(t)) & =\int_{\Omega_{h}} \nabla v \cdot \nabla v_{t} d x+a \int_{\Omega_{h}} v_{t} v d x-\int_{\Omega_{h}} v_{t} f(v) d x-\int_{\partial \Omega_{h}} g(\gamma(v)) v_{t} d S \\
& =-\int_{\Omega_{h}} v_{t} v_{t} d x \\
& =-\left\|v_{t}\right\|_{L^{2}\left(\Omega_{h}\right)^{\prime}}^{2}
\end{aligned}
$$


onde usamos a formulação fraca do problema. Logo, $W_{h}$ é decrescente ao longo das soluções de (2.12).

Além disso, se $v(t)$ é uma solução de (2.12) e $W_{h}(v(t))=W_{h}\left(v_{0}\right), \forall t \geq 0$, com $v_{0} \in H^{1}\left(\Omega_{h}\right)$, então

$$
\frac{d}{d t} W_{h}(v(t))=\frac{d}{d t} W_{h}\left(v_{0}\right)=0,
$$

o que significa que $\left\|v_{t}\right\|_{L^{2}\left(\Omega_{h}\right)}^{2}=0$ e $v$ é um equilíbrio de 2.12 .

Provamos assim que $W_{h}$ é uma função de Lyapunov para o fluxo gerado por (2.12). Resta as estimativas para $W_{h}$.

Por 2.9), existem $\varepsilon_{f}>0$ e $M\left(\varepsilon_{f}\right)>0$ tais que $\frac{f(s)}{s}-c_{0} \leq \varepsilon_{f}$ para $|s|>M\left(\varepsilon_{f}\right)$. E, como f é contínua e $|s| \leq M\left(\varepsilon_{f}\right)$ é compacto, vale que $f(s) \leq k_{f}$ para $|s| \leq M\left(\varepsilon_{f}\right)$, sendo $k_{f}$ uma constante. Consequentemente, se $s>0, f(s) \leq\left(\varepsilon_{f}+c_{0}\right) s+k_{f}$ e,

$$
\int_{\Omega_{h}} F(v) d x=\int_{\Omega_{h}}\left(\int_{0}^{v} f(s) d s\right) d x \leq \int_{\Omega_{h}}\left(\int_{0}^{v} \varepsilon_{f} s+c_{0} s+k_{f} d s\right) d x \leq \frac{c_{0}}{2} \int_{\Omega_{h}}|v|^{2} d x+k_{0} .
$$

Podemos repetir o mesmo raciocínio para $s<0$. E, por $(2.10)$, existem $\varepsilon_{g}>0$ e $N\left(\varepsilon_{g}\right)>0$ tais que $\frac{g(s)}{s}-d_{0}^{\prime} \leq \varepsilon_{g}$ para $|s|>N\left(\varepsilon_{g}\right)$. Analogamente,

$$
\int_{\partial \Omega_{h}} G(\gamma(v)) d S \leq \frac{d_{0}^{\prime}}{2} \int_{\partial \Omega_{h}}|\gamma(v)|^{2} d S+k_{0}^{\prime} .
$$

Daí,

$$
\begin{aligned}
W_{h}(v) & \leq \frac{1}{2} \int_{\Omega_{h}}|\nabla v|^{2} d x+\frac{a}{2} \int_{\Omega_{h}}|v|^{2} d x+\frac{\left|c_{0}\right|}{2} \int_{\Omega_{h}}|v|^{2} d x+\left|k_{0}\right|+\frac{\left|d_{0}^{\prime}\right|}{2} \int_{\partial \Omega_{h}}|\gamma(v)|^{2} d S+\left|k_{0}^{\prime}\right| \\
& \leq \frac{1}{2}\|\nabla v\|_{L^{2}\left(\Omega_{h}\right)}^{2}+\frac{a}{2}\|v\|_{L^{2}\left(\Omega_{h}\right)}^{2}+\frac{\left|c_{0}\right|}{2}\|v\|_{L^{2}\left(\Omega_{h}\right)}^{2}+\left|k_{0}\right|+\frac{\left|d_{0}^{\prime}\right|}{2}\|\gamma(v)\|_{L^{2}\left(\partial \Omega_{h}\right)}^{2}+\left|k_{0}^{\prime}\right| \\
& \leq \frac{1}{2}\|\nabla v\|_{L^{2}\left(\Omega_{h}\right)}^{2}+\frac{a+\left|c_{0}\right|}{2}\|v\|_{L^{2}\left(\Omega_{h}\right)}^{2}+\left|k_{0}\right|+\frac{\left|d_{0}^{\prime}\right|}{2}\|v\|_{L^{2}\left(\Omega_{h}\right)}^{2}+\left|k_{0}^{\prime}\right| \\
& \leq K_{1}\|v\|_{H^{1}\left(\Omega_{h}\right)}^{2}+K_{2}
\end{aligned}
$$

com $K_{1}$ e $K_{2}$ constantes dependendo essencialmente das não linearidades, o que prova (2.14).

Por outro lado,

$$
\begin{aligned}
W_{h}(v) & \geq \frac{1}{2} \int_{\Omega_{h}}|\nabla v|^{2} d x+\frac{a}{2} \int_{\Omega_{h}}|v|^{2} d x-\frac{c_{0}}{2} \int_{\Omega_{h}}|v|^{2} d x-k_{0}-\frac{d_{0}^{\prime}}{2} \int_{\partial \Omega_{h}}|\gamma(v)|^{2} d S-k_{0}^{\prime} \\
& \geq \frac{1}{2} \int_{\Omega_{h}}|\nabla v|^{2} d x+\frac{a-c_{0}}{2} \int_{\Omega_{h}}|v|^{2} d x-\frac{d_{0}^{\prime}}{2} \int_{\partial \Omega_{h}}|\gamma(v)|^{2} d S-\left(k_{0}+k_{0}^{\prime}\right) .
\end{aligned}
$$

Como $d_{0}>d_{0}^{\prime}$, podemos escolher $d_{0}^{\prime \prime} \neq 0$ tal que

$$
d_{0}>d_{0}^{\prime \prime}>d_{0}^{\prime} \text { e }\left(\frac{d_{0}}{d_{0}^{\prime \prime}}-1\right) \frac{a-c_{0}}{2}+\frac{\lambda_{0}}{2}>0,
$$


onde $\lambda_{0}$ é o primeiro autovalor de 2.11) e,

$$
\begin{aligned}
W_{h}(v) & \geq \frac{1}{2} \int_{\Omega_{h}}|\nabla v|^{2} d x+\frac{a-c_{0}}{2} \int_{\Omega_{h}}|v|^{2} d x-\frac{d_{0}^{\prime \prime}}{2} \int_{\partial \Omega_{h}}|\gamma(v)|^{2} d S-\left(k_{0}+k_{0}^{\prime}\right) \\
& =\frac{d_{0}^{\prime \prime}}{d_{0}}\left[\frac{d_{0}}{d_{0}^{\prime \prime}} \frac{1}{2} \int_{\Omega_{h}}|\nabla v|^{2} d x+\frac{d_{0}}{d_{0}^{\prime \prime}} \frac{\left(a-c_{0}\right)}{2} \int_{\Omega_{h}}|v|^{2} d x-\frac{d_{0}}{2} \int_{\partial \Omega_{h}}|\gamma(v)|^{2} d S-\frac{d_{0}}{d_{0}^{\prime \prime}}\left(k_{0}+k_{0}^{\prime}\right)\right] \\
& =\frac{d_{0}^{\prime \prime}}{d_{0}}\left[\left(\frac{d_{0}}{d_{0}^{\prime \prime}}-1\right) \frac{1}{2} \int_{\Omega_{h}}|\nabla v|^{2} d x+\frac{1}{2} \int_{\Omega_{h}}|\nabla v|^{2} d x+\left(\frac{d_{0}}{d_{0}^{\prime \prime}}-1\right) \frac{\left(a-c_{0}\right)}{2} \int_{\Omega_{h}}|v|^{2} d x\right] \\
& +\frac{d_{0}^{\prime \prime}}{d_{0}}\left[\frac{\left(a-c_{0}\right)}{2} \int_{\Omega_{h}}|v|^{2} d x-\frac{d_{0}}{2} \int_{\partial \Omega_{h}}|\gamma(v)|^{2} d S-\frac{d_{0}}{d_{0}^{\prime \prime}}\left(k_{0}+k_{0}^{\prime}\right)\right] \\
& \geq \frac{d_{0}^{\prime \prime}}{d_{0}}\left[\left(\frac{d_{0}}{d_{0}^{\prime \prime}}-1\right) \frac{1}{2} \int_{\Omega_{h}}|\nabla v|^{2} d x+\left(\left(\frac{d_{0}}{d_{0}^{\prime \prime}}-1\right) \frac{\left(a-c_{0}\right)}{2}+\frac{\lambda_{0}}{2}\right) \int_{\Omega_{h}}|v|^{2} d x-\frac{d_{0}}{d_{0}^{\prime \prime}}\left(k_{0}+k_{0}^{\prime}\right)\right] .
\end{aligned}
$$

Vale observar que, para obter a última desigualdade usamos que $\lambda_{0}=\inf _{u \in H^{1}\left(\Omega_{h}\right)} \frac{\left\langle-\Delta u+\left(a-c_{0}\right) u, u>\right.}{\|u\|_{L^{2}\left(\Omega_{h}\right)}}$. Assim, $W_{h}(v)$ é limitado inferiormente por uma constante positiva dependendo das não linearidades e do primeiro autovalor de 2.11 vezes $\|v\|_{H^{1}\left(\Omega_{h}\right)}^{2}$ mais uma constante que depende das não linearidades. Portanto, $\left|W_{h}(v)\right| \rightarrow \infty$ quando $\|v\|_{H^{1}\left(\Omega_{h}\right)} \rightarrow \infty$.

Lema 2.5.2 Para $W_{h}: H^{1}\left(\Omega_{h}\right) \rightarrow \mathbb{R}$ definido como em 2.13, existem constantes $K_{1}(h)$ e $K_{2}(h)$ tais que,

$$
K_{1}(h)|W(u)| \leq\left|W_{h}\left(u \circ h^{-1}\right)\right| \leq K_{2}(h)|W(u)|, \quad \forall u \in H^{1}(\Omega),
$$

com $K_{1}(h), K_{2}(h) \rightarrow 1$ quando $h \rightarrow i_{\Omega}$, ou seja, os funcionais de Lyapunov $W_{h}$ nas regiões perturbadas aproximam o funcional $W=W_{i_{\Omega}}$ quando $h \rightarrow i_{\Omega}$.

\section{Demonstração:}

Um caso particular foi provado no Lema 4.3 em Oliveira, Pereira e Pereira (2005), quando o funcional de Lyapunov consiste apenas da primeira e da terceira parcelas de $W_{h}$. Para os demais termos na expressão 2.13), por exemplo,

$$
\frac{a}{2} \int_{\Omega_{h}}|v|^{2} d y=\frac{a}{2} \int_{\Omega_{h}}\left|u \circ h^{-1}\right|^{2} d y=\frac{a}{2} \int_{\Omega}|u|^{2}\left|J_{h}\right| d x \leq \max _{x \in \bar{\Omega}}\left\{\left|J_{h}\right|\right\} \frac{a}{2} \int_{\Omega}|u|^{2} d x,
$$

$\operatorname{com}\left|J_{h}\right| \rightarrow 1$ à medida que $h \rightarrow i_{\Omega}$ em $C^{2}\left(\Omega, \mathbb{R}^{2}\right)$. E, para obter a limitação inferior, poderíamos usar analogamente $\min _{x \in \bar{\Omega}}\left\{\left|J_{h}\right|\right\}$.

Neste ponto, definamos $V_{h}: H^{1}(\Omega) \rightarrow \mathbb{R}$ por

$$
V_{h}(u)=W_{h}\left(u \circ h^{-1}\right)
$$


Lema 2.5.3 O funcional definido em 2.15) é um funcional de Lyapunov para (2.5) $\operatorname{com} \eta=\frac{1}{2} e-\frac{1}{2}<\beta<$ $-\frac{1}{4}$ e valem as seguintes estimativas:

(a) $K_{1}\|u\|_{H^{1}(\Omega)}^{2}-K_{2} \leq V_{h}(u) \leq K_{1}\|u\|_{H^{1}(\Omega)}^{2}+K_{2}$, para constantes $K_{1}, K_{2}$;

(b) $K_{1}(h)|V(u)| \leq\left|V_{h}(u)\right| \leq K_{2}(h)|V(u)|, \forall u \in H^{1}(\Omega)$ com $K_{1}(h), K_{2}(h) \rightarrow 1$ quando $h \rightarrow i_{\Omega}$.

\section{Demonstração:}

Tais propriedades seguem daquelas para $W_{h}$ e do fato de $h^{*-1}: H^{1}(\Omega) \rightarrow H^{1}\left(\Omega_{h}\right)$ ser um isomorfismo que leva soluções de (2.5) em soluções de (2.12).

Teorema 2.5.4 Sejam $\beta, \eta, f, g$, $h$ tais como no Teorema 2.4.6 e suponha que $f$ e g satisfazem as hipóteses dissipativas (2.9) e 2.10) respectivamente. Então, as soluções de (2.5) estão globalmente definidas.

\section{Demonstração:}

Consideremos primeiramente o caso $\eta=\frac{1}{2}$. Pelo Teorema 2.4.6, para cada $\left(t_{0}, u_{0}\right) \in \mathbb{R} \times H^{1}(\Omega)$, existe $T=T\left(t_{0}, u_{0}\right)$ tal que o problema (2.5) tem uma única solução $u$ em $\left(t_{0}, t_{0}+T\right) \operatorname{com} u\left(t_{0}\right)=u_{0}$. Sabemos também que $\left(H_{h,-1 / 2}\right)_{\beta}$ leva limitados de $X_{h}^{\eta}=H^{1}(\Omega)$ em conjuntos limitados de $X_{h}^{\beta}$.

Se $T<\infty$, pelo Teorema 3.3.4 em Henry (1981), existe uma sequência $t_{n} \rightarrow T^{-}$tal que $\|$ $u\left(t_{n}\right) \|_{H^{1}(\Omega)} \rightarrow \infty$ e, consequentemente, $\left|V_{h}\left(u\left(t_{n}\right)\right)\right| \rightarrow \infty$, usando (a) do Lema 2.5.3, o que é uma contradição com o fato de $V_{h}$ ser decrescente ao longo das órbitas.

Para os demais casos, se $\eta<\frac{1}{2}$, temos $X_{h}^{\frac{1}{2}} \subset X_{h}^{\eta}$ e dado $u_{0} \in X_{h^{\prime}}^{\eta}$ existe uma solução local $u(t), t_{0}<t<T$. Por regularidade, para $t_{0}<t^{\prime}<T, T_{h}\left(t^{\prime}\right) u_{0} \in X_{h}^{\frac{1}{2}}$ e tomando este como ponto inicial $u_{0}^{\prime}$, provamos que existe uma solução global passando por $u_{0}^{\prime}$. Segue da unicidade o desejado. Finalmente, se $\eta>\frac{1}{2}, X_{h}^{\eta} \subset X_{h}^{\frac{1}{2}}$ de modo que tomando $u_{0} \in X_{h}^{\eta}, u_{0}$ também pertence à $X_{h}^{\frac{1}{2}}$ e então, existe uma solução global passando por tal ponto, a qual coincide com a solução local em $X_{h}^{\eta}$ por unicidade.

\subsection{Existência de um atrator global}

Demonstramos nesta seção que $T_{h, \eta, \beta}\left(t, t_{0}, u\right)$, o fluxo gerado por (2.5), admite um atrator global começando pelo caso $\eta=\frac{1}{2}$. 
Lembramos que um semigrupo fortemente contínuo $T(t): X \rightarrow X, t \geq 0$, de classe $C^{r}$, com $r \geq 1$, é um sistema gradiente se,

(i) Cada órbita positiva limitada é pré-compacta.

(ii) Existe uma função de Lyapunov para $T(t)$, isto é, existe uma função contínua $V: X \rightarrow \mathbb{R}$ com as seguintes propriedades:

(1) $V(x)$ é limitada inferiormente;

(2) $V(x) \rightarrow \infty$ quando $|x| \rightarrow \infty$;

(3) $V(T(t) x)$ é decrescente em $t$ para cada $x \in X$;

(4) Se $x$ é tal que $T(t) x$ está definida para $t \in \mathbb{R}$ e $V(T(t) x)=V(x)$ para $t \in \mathbb{R}$, então $x$ é um ponto de equilíbrio.

Lema 2.6.1 O semigrupo não linear $T_{h, \eta, \beta}(t)$ gerado por (2.5) $\operatorname{com} \eta=\frac{1}{2} e-\frac{1}{2}<\beta<-\frac{1}{4}$ em $X_{h}^{\frac{1}{2}}=H^{1}(\Omega)$ é um sistema gradiente.

\section{Demonstração:}

Sabemos que $V_{h}$ definido em 2.15) é um funcional de Lyapunov para o semigrupo.

Além disso, $\left(H_{h,-1 / 2}\right)_{\beta}$ leva subconjuntos limitados de $H^{1}(\Omega)$ em limitados de $X_{h}^{\beta}$ e como podemos escrever $\left(-A_{h,-1 / 2}\right)_{\beta}=\left(-A_{h,-1 / 2}\right)^{-\delta}\left(-A_{h,-1 / 2}\right)\left(-A_{h,-1 / 2}\right)^{\delta}, \beta=-\frac{1}{2}+\delta, 0<\delta<\frac{1}{4}$, sendo $\left(-A_{h,-1 / 2}\right)^{\delta}$ uma isometria, segue que $\left(-A_{h,-1 / 2}\right)_{\beta}$ tem resolvente compacto. Assim, de acordo com o Teorema 1.4.3, as órbitas positivas limitadas são pré-compactas.

Logo, temos que $T_{h, \eta, \beta}(t)$ é um sistema gradiente.

Lema 2.6.2 Suponha que $f$ e $g$ satisfazem as condições do Teorema 2.4.6 e as hipóteses dissipativas (2.9) $e$ (2.10) respectivamente. Então, o conjunto dos pontos de equilíbrio do sistema gerado por (2.12) é uniformemente limitado em $h$ na norma $H^{1}\left(\Omega_{h}\right)$.

\section{Demonstração:}

Os equilíbrios de (2.12) são as soluções do problema 


$$
\left\{\begin{array}{l}
\Delta u(x)-a u(x)+f(u(x))=0, x \in \Omega_{h} \\
\frac{\partial u}{\partial N}(x)=g(u(x)), x \in \partial \Omega_{h} .
\end{array}\right.
$$

Multiplicando por $u$ e integrando, obtemos,

$$
\begin{aligned}
0 & =\int_{\Omega_{h}} u \Delta u d x-a \int_{\Omega_{h}}|u|^{2} d x+\int_{\Omega_{h}} u f(u) d x \\
& =-\int_{\Omega_{h}}|\nabla u|^{2} d x+\int_{\partial \Omega_{h}} u g(u) d S-a \int_{\Omega_{h}}|u|^{2} d x+\int_{\Omega_{h}} u f(u) d x .
\end{aligned}
$$

Por 2.9) e 2.10), para qualquer $\delta>0$, existem constantes $M_{f}$ e $M_{g}$ tais que $f(s) s \leq\left(\delta+c_{0}\right) s^{2}$ se $|s|>M_{f}$ e $g(s) s \leq\left(\delta+d_{0}^{\prime}\right) s^{2}$ se $|s|>M_{g}$. Escolhendo $|s|>M=\max \left\{M_{f}, M_{g}\right\}$, ambas as desigualdades valem simultaneamente. Além disso, existem constantes $C_{f}, C_{g} \operatorname{com} f(s) s \leq C_{f}$ e $g(s) s \leq C_{g}$ para $|s| \leq M$. Se fizermos $K_{\delta}=\max \left\{C_{f}, C_{g}\right\}$, então para todo $u \in \mathbb{R}$,

$$
f(u) u \leq\left(c_{0}+\delta\right) u^{2}+K_{\delta} \text { e } g(u) u \leq\left(d_{0}^{\prime}+\delta\right) u^{2}+K_{\delta}
$$

Aplicando isso em (2.16),

$$
\begin{aligned}
\int_{\Omega_{h}}|\nabla u|^{2} d x & \leq-a \int_{\Omega_{h}}|u|^{2} d x+\left(c_{0}+\delta\right) \int_{\Omega_{h}} u^{2} d x+K_{\delta}\left|\Omega_{h}\right|+\left(d_{0}^{\prime}+\delta\right) \int_{\partial \Omega_{h}} u^{2} d S+K_{\delta}\left|\partial \Omega_{h}\right| \\
& \leq-a \int_{\Omega_{h}}|u|^{2} d x+\left(c_{0}+\delta\right) \int_{\Omega_{h}} u^{2} d x+\left(d_{0}^{\prime}+\delta\right) \int_{\partial \Omega_{h}} u^{2} d S+K_{\delta}(|\Omega|+|\partial \Omega|) .(2.17)
\end{aligned}
$$

Como o primeiro autovalor $\lambda_{0}(h)$ de 2.11) é positivo, temos que

$$
\lambda_{0}(h)=\inf _{u \in H^{1}\left(\Omega_{h}\right)} \frac{<-\Delta u+\left(a-c_{0}\right) u, u>}{\|u\|_{L^{2}\left(\Omega_{h}\right)}^{2}} .
$$

Ao desenvolver essa expressão, encontramos

$$
\lambda_{0}(h) \int_{\Omega_{h}}|u|^{2} d x \leq \int_{\Omega_{h}}|\nabla u|^{2} d x-d_{0} \int_{\partial \Omega_{h}}|u|^{2} d S+\left(a-c_{0}\right) \int_{\Omega_{h}}|u|^{2} d x .
$$

Reunindo as desigualdades (2.17) e (2.18), obtemos

$$
\lambda_{0}(h) \int_{\Omega_{h}}|u|^{2} d x \leq \delta \int_{\Omega_{h}} u^{2} d x+\left(d_{0}^{\prime}-d_{0}+\delta\right) \int_{\partial \Omega_{h}} u^{2} d S+K_{\delta}(|\Omega|+|\partial \Omega|) .
$$

Agora, tomando $\delta<\min \left\{\lambda_{0}(h), d_{0}-d_{0}^{\prime}\right\}$ e estabelecendo $l_{\delta}=\min \left\{\lambda_{0}(h)-\delta,\left(d_{0}-d_{0}^{\prime}\right)-\delta\right\}$, segue de (2.19) que,

$$
\int_{\Omega_{h}} u^{2} d x+\int_{\partial \Omega_{h}} u^{2} d S \leq \frac{K_{\delta}(|\Omega|+|\partial \Omega|)}{l_{\delta}}
$$

Por fim, empregamos isso em (2.17),

$$
\begin{aligned}
\int_{\Omega_{h}}|\nabla u|^{2} d x & \leq\left(c_{0}+\delta\right) \int_{\Omega_{h}} u^{2} d x+\left(d_{0}^{\prime}+\delta\right) \int_{\partial \Omega_{h}} u^{2} d S+K_{\delta}(|\Omega|+|\partial \Omega|) \\
& \leq\left(c_{0}+d_{0}^{\prime}+2 \delta\right) \frac{K_{\delta}(|\Omega|+|\partial \Omega|)}{l_{\delta}}+K_{\delta}(|\Omega|+|\partial \Omega|)
\end{aligned}
$$


onde usamos que $a>0$ e supomos que $c_{0}+\delta$ e $d_{0}^{\prime}+\delta$ são ambos positivos. Caso sejam negativos, em (2.21), ficaria apenas a última parcela e se os sinais fossem contrários, manteríamos somente o positivo. O resultado vem de (2.20) e (2.21), observando-se que as constantes presentes nessas estimativas dependem basicamente de $\lambda_{0}(h)$, que é uma função contínua de $h$, sendo limitada para $h$ próxima de $i_{\Omega}$.

Corolário 2.6.3 Suponha que $f$ e g satisfazem as condições do Lema 2.6.2. O conjunto de equilíbrios $E_{h}$ do sistema gerado por (2.5) é uniformemente limitado em $h$ na norma $H^{1}(\Omega)$, para $\left\|h-i_{\Omega}\right\|_{C^{2}\left(\Omega, \mathbb{R}^{2}\right)}<\epsilon_{0}$.

\section{Demonstração:}

Como $u$ é um equilíbrio de (2.5) se, e somente se, $v=h^{*-1} u$ é um equilíbrio de 2.12, com $h^{*}$ um isomorfismo, o resultado segue do Lema 2.6.2.

Teorema 2.6.4 Suponha que $f$ e $g$ satisfazem as condições do Teorema 2.4.6 com $\eta=\frac{1}{2}$ e as hipóteses dissipativas 2.9p e 2.10 respectivamente. Então, para h suficientemente próxima de $i_{\Omega}$, ofluxo $T_{h, \frac{1}{2}, \beta}(t, u)=$ $T_{h}(t, u)$ gerado por (2.5) tem um atrator global em $X_{h}^{\frac{1}{2}}=H^{1}(\Omega)$.

\section{Demonstração:}

De acordo com o Teorema 3.8.5 em Hale (2000), basta provar que o semigrupo é assintoticamente compacto. Seja $B$ um conjunto não-vazio, fechado, limitado de $X_{h}^{\frac{1}{2}}=H^{1}(\Omega)$ com $T_{h}(t, u) B \subset B, \forall t \geq 0$.

Considere $J=\overline{T_{h}(1) B}$. Então, $J \subset B$. Pela propriedade de semigrupo, para $t>1, T_{h}(t) B=$ $T_{h}(1) T_{h}(t-1) B$ e $T_{h}(1) T_{h}(t-1) B \subset T_{h}(1) B \subset J$ donde $J$ atrai $B$ (de fato, absorve). Falta verificarmos que $J$ é compacto.

A idéia é usar o Teorema 1.2.18, pois se soubermos que $J$ é limitado em $X_{h}^{\frac{1}{2}+\epsilon}, \operatorname{com} \epsilon>0$, então, como a imersão $X_{h}^{\frac{1}{2}+\epsilon} \subset X_{h}^{\frac{1}{2}}=H^{1}(\Omega)$ é compacta, resulta que J é um pré-compacto de $H^{1}(\Omega)$. Somando-se a isso o fato de $J$ ser fechado, temos o desejado.

Sem perda de generalidade, suponhamos $\mathfrak{R}\left(\sigma\left(\left(-A_{h,-1 / 2}\right)_{\beta}\right)\right)>\omega>0$. Se $j=T_{h}(1) b, b \in B$ e 
$\|b\|_{X_{h}^{\frac{1}{2}}} \leq N$

$$
\begin{aligned}
\|j\|_{X_{h}^{\frac{1}{2}+\epsilon}} & \leq\left\|e^{-\left(-A_{h,-1 / 2}\right)_{\beta}} b\right\|_{X_{h}^{\frac{1}{2}+\epsilon}}+\left\|\int_{0}^{1} e^{-\left(-A_{h,-1 / 2}\right)_{\beta}(1-s)}\left(H_{h,-1 / 2}\right)_{\beta}\left(T_{h}(s)\right) d s\right\|_{X_{h}^{\frac{1}{2}+\epsilon}} \\
& \leq\left\|\left(\left(-A_{h,-1 / 2}\right)_{\beta}\right)^{\epsilon} e^{-\left(-A_{h,-1 / 2}\right)_{\beta}} b\right\|_{X_{h}^{\frac{1}{2}}}+\int_{0}^{1}\left\|\left(\left(-A_{h,-1 / 2}\right)_{\beta}\right)^{1-\epsilon} e^{-\left(-A_{h,-1 / 2}\right)_{\beta}(1-s)}\left(H_{h,-1 / 2}\right)_{\beta}\left(T_{h}(s)\right)\right\|_{X_{h}^{-\frac{1}{2}+2 \epsilon}} d s \\
& \leq C_{\epsilon} e^{-\omega}\|b\|_{X_{h}^{\frac{1}{2}}}+\int_{0}^{1} C_{1-\epsilon}(1-s)^{-(1-\epsilon)} e^{-\omega(1-s)}\left\|\left(H_{h,-1 / 2}\right)_{\beta}\left(T_{h}(s)\right)\right\|_{X_{h}^{-\frac{1}{2}+2 \epsilon}} d s \\
& \leq C_{\epsilon} e^{-\omega} N+C_{1-\epsilon} L \int_{0}^{1}(1-s)^{-(1-\epsilon)} e^{-\omega(1-s)} d s
\end{aligned}
$$

onde $L$ vem de $\left(H_{h,-1 / 2}\right)_{\beta}$ ser localmente Lipschitz e aplicamos o Teorema 1.2.9. A última expressão nos mostra que $T_{h}(1) B$ é limitado em $X_{h}^{\frac{1}{2}+\epsilon}$ e, portanto, seu fecho, J, também o é.

Teorema 2.6.5 Suponha que estejam satisfeitas as condições do Teorema 2.4.6 e as hipóteses dissipativas (2.9) e 2.10). Então, para $h$ suficientemente próxima de $i_{\Omega}$, o semigrupo $T_{h, \eta, \beta}(t, u)$ gerado por (2.5) tem um atrator global $\mathcal{A}_{h}$ em $X_{h}^{\eta}$. Além disso, $\mathcal{A}_{h}$ não depende de $\eta$ ou $\beta$.

\section{Demonstração:}

Suponhamos primeiro que $\eta=\eta_{0}<\frac{1}{2}$ e seja $B$ um limitado de $X_{h}^{\eta}$. Podemos admitir que $B=B_{R}$, a bola aberta de raio $R$ e centro na origem de $X_{h}^{\eta}$. Conforme vimos, existe $N$ tal que

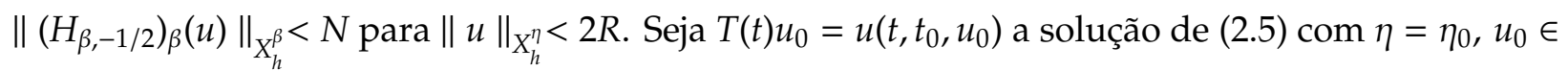
$B_{R}$ e seja $\omega$ tal que $\mathfrak{R}\left(\sigma\left(\left(-A_{h,-1 / 2}\right)_{\beta}\right)\right)>\omega>0$. Enquanto $\left\|u\left(t, t_{0}, u_{0}\right)\right\|_{X_{h}^{\eta \eta}}<2 R$, temos pela Fórmula de Variação das Constantes,

$$
\begin{aligned}
\left\|u\left(t, t_{0}, u_{0}\right)\right\|_{X_{h}^{\eta}} & \leq\left\|\left(\left(-A_{h,-1 / 2}\right)_{\beta}\right)^{\eta-\beta} e^{-\left(-A_{h,-1 / 2}\right)_{\beta}\left(t-t_{0}\right)} u_{0}\right\|_{X_{h}^{\beta}} \\
& +\int_{t_{0}}^{t}\left\|\left(\left(-A_{h,-1 / 2}\right)_{\beta}\right)^{\eta-\beta} e^{-\left(-A_{h,-1 / 2}\right)_{\beta}(t-s)}\left(H_{h,-1 / 2}\right)_{\beta} u(s)\right\|_{X_{h}^{\beta}} d s \\
& \leq C_{\eta-\beta}\left(t-t_{0}\right)^{\beta-\eta} e^{-\omega\left(t-t_{0}\right)}\left\|u_{0}\right\|_{X_{h}^{\beta}}+N C_{\eta-\beta} \int_{t_{0}}^{t}(t-s)^{(\beta-\eta)} e^{-\omega(t-s)} d s,
\end{aligned}
$$

onde usamos o Teorema 1.2.9. Consideremos $T=\sup \left\{t \geq t_{0} \mid u\left(s, t_{0}, u_{0}\right) \in B_{2 R}, \forall s \leq t\right\}$ e $\delta>t_{0}$ tal que o lado direito desta última expressão (2.22) seja menor $2 R, \forall t \in\left(t_{0}, \delta\right)$. Segue que $T \geq \delta$ e as soluções com condições iniciais na bola de raio $R$ em $X_{h}^{\eta}$ permanecem na bola de raio $2 R$ para 
$t_{0} \leq t \leq T$. Agora,

$$
\begin{aligned}
\left\|u\left(t, t_{0}, u_{0}\right)\right\|_{X_{h}^{\frac{1}{2}}} & \leq\left\|e^{-\left(-A_{h,-1 / 2}\right)_{\beta}\left(t-t_{0}\right)} u_{0}\right\|_{X_{h}^{\frac{1}{2}}}+\int_{t_{0}}^{t}\left\|e^{-\left(-A_{h,-1 / 2}\right)_{\beta}(t-s)}\left(H_{h,-1 / 2}\right)_{\beta} u(s)\right\|_{X_{h}^{\frac{1}{2}}} d s \\
& \leq\left\|\left(\left(-A_{h,-1 / 2}\right)_{\beta}\right)^{\frac{1}{2}-\eta} e^{-\left(-A_{h,-1 / 2}\right)_{\beta}\left(t-t_{0}\right)} u_{0}\right\|_{X_{h}^{\eta}} \\
& +\int_{t_{0}}^{t}\left\|\left(\left(-A_{h,-1 / 2}\right)_{\beta}\right)^{\frac{1}{2}-\beta} e^{-\left(-A_{h,-1 / 2}\right)_{\beta}(t-s)}\left(H_{h,-\frac{1}{2}}\right)_{\beta} u(s)\right\|_{X_{h}^{\beta}} d s \\
& \leq C_{\frac{1}{2}-\eta}\left(t-t_{0}\right)^{-\left(\frac{1}{2}-\eta\right)} e^{-\omega\left(t-t_{0}\right)}\left\|u_{0}\right\|_{X_{h}^{\eta}}+N C_{\frac{1}{2}-\beta} \int_{t_{0}}^{t}(t-s)^{-\left(\frac{1}{2}-\beta\right)} e^{-\omega(t-s)} d s,
\end{aligned}
$$

o que significa que $T(t) B_{R}$ está em um limitado de $X_{h}^{\frac{1}{2}}$ para $t_{0}<t \leq T$. Pelo Teorema 2.6.4, o atrator global de (2.5) $\operatorname{com} \eta=\frac{1}{2}$ atrai $T(t) B_{R}$ na norma $X_{h}^{\frac{1}{2}}, t_{0}<t<T$. Como $X_{h}^{\frac{1}{2}} \subset X_{h^{\prime}}^{\eta} \mathcal{A}_{h}$ atrai $B_{R}$ na norma de $X_{h}^{\eta}$. Uma vez que $\mathcal{A}_{h}$ é invariante, este deve ser o atrator de (2.5) $\operatorname{com} \eta=\eta_{0}$.

Suponhamos agora, $\frac{1}{2}<\eta=\eta_{0}<\beta+1$. Temos $X_{h}^{\eta} \subset X_{h}^{\frac{1}{2}}$ continuamente de acordo com o Teorema 1.2.18. Assim, um conjunto limitado $B$ de $X_{h}^{\eta}$ é também limitado em $X_{h}^{\frac{1}{2}}$, sendo atraído por $\mathcal{A}_{h}$ de (2.5) com $\eta=\frac{1}{2}$ em $X_{h}^{\frac{1}{2}}$ sob a ação de $T_{h, \frac{1}{2}, \beta}(t)=T_{\frac{1}{2}}(t)$, o qual coincide com o fluxo em $X_{h}^{\eta}$. Mostraremos a seguir que $T_{\frac{1}{2}}(t)$ é contínuo de $X_{h}^{\frac{1}{2}}$ em $X_{h}^{\eta}$ para $t>0$.

Sejam $u_{1}, u_{2} \in X_{h}^{\frac{1}{2}}$ e $u_{i}\left(t, t_{0}, u_{i}\right)=T_{\frac{1}{2}}(t) u_{i}, i=1,2$.

$$
\begin{aligned}
\left\|u\left(t, t_{0}, u_{1}\right)-u\left(t, t_{0}, u_{2}\right)\right\|_{X_{h}^{\eta}} & \leq\left\|e^{-\left(-A_{h,-1 / 2}\right)_{\beta}\left(t-t_{0}\right)}\left(u_{1}-u_{2}\right)\right\|_{X_{h}^{\eta}} \\
& +\int_{t_{0}}^{t}\left\|e^{-\left(-A_{h,-1 / 2}\right)_{\beta}(t-s)}\left(\left(H_{h,-1 / 2}\right)_{\beta}\left(u_{1}(s)\right)-\left(H_{h,-1 / 2}\right)_{\beta}\left(u_{2}(s)\right)\right)\right\|_{X_{h}^{\eta}} d s \\
& \leq\left\|\left(\left(-A_{h,-1 / 2}\right)_{\beta}\right)^{\eta-\frac{1}{2}} e^{-\left(-A_{h,-1 / 2}\right)_{\beta}\left(t-t_{0}\right)}\left(u_{1}-u_{2}\right)\right\|_{X_{h}^{\frac{1}{2}}} \\
& +\int_{t_{0}}^{t}\left\|\left(\left(-A_{h,-1 / 2}\right)_{\beta}\right)^{\eta-\beta} e^{-\left(-A_{h,-1 / 2}\right)_{\beta}(t-s)}\left(\left(H_{h,-1 / 2}\right)_{\beta}\left(u_{1}(s)\right)-\left(H_{h,-1 / 2}\right)_{\beta}\left(u_{2}(s)\right)\right)\right\|_{X_{h}^{\beta}} d s \\
& \leq C_{\eta-\frac{1}{2}}\left(t-t_{0}\right)^{\frac{1}{2}-\eta} e^{-\omega\left(t-t_{0}\right)}\left\|u_{1}-u_{2}\right\|_{X_{h}^{\frac{1}{2}}} \\
& +L_{\beta, \frac{1}{2}} C_{\eta-\beta} \int_{t_{0}}^{t}(t-s)^{\beta-\eta} e^{-\omega(t-s)}\left\|u_{1}(s)-u_{2}(s)\right\|_{X_{h}^{\frac{1}{2}}} d s,
\end{aligned}
$$

sendo $L_{\beta, \frac{1}{2}}$ a constante de Lipschitz local de $\left(H_{h,-1 / 2}\right)_{\beta}$ e as demais constantes vem do Teorema 1.2.9.

Aplicando a Desigualdade de Gronwall, Teorema 7.1.1 em Henry (1981), obtemos

$$
\begin{aligned}
\left\|u\left(t, t_{0}, u_{1}\right)-u\left(t, t_{0}, u_{2}\right)\right\|_{X_{h}^{\eta}} & \leq C_{\eta-\frac{1}{2}}\left(t-t_{0}\right)^{-\eta+\frac{1}{2}} e^{-\omega\left(t-t_{0}\right)}\left\|u_{1}-u_{2}\right\|_{X_{h}^{\frac{1}{2}}} \\
& +\gamma \int_{t_{0}}^{t} E_{\frac{3}{2}-\eta}^{\prime}(\gamma(t-s)) C_{\eta-\frac{1}{2}}\left(s-t_{0}\right)^{-\eta+\frac{1}{2}} e^{-\omega\left(s-t_{0}\right)}\left\|u_{1}-u_{2}\right\|_{X_{h}^{\frac{1}{2}}} d s,
\end{aligned}
$$

onde $\gamma=\left(L_{\beta, \frac{1}{2}} C_{\eta-\frac{1}{2}} \max _{t \leq s \leq t_{0}}\left\{e^{-\omega(t-s)}\right\} \Gamma\left(\frac{3}{2}-\eta\right)\right)^{\frac{1}{\frac{3}{2}-\eta}}$ e $E_{\frac{3}{2}-\eta}(z)=\sum_{n=0}^{\infty} \frac{z^{n\left(\frac{3}{2}-\eta\right)}}{\Gamma\left(n\left(\frac{3}{2}-\eta\right)+1\right)}$ e, segue a continuidade. 
Isso nos permite concluir que se $V_{\delta}$ é uma vizinhança de $\mathcal{A}_{h}$ em $X_{h}^{\frac{1}{2}}$ que contém $T_{\frac{1}{2}}(t)(B)=T_{\eta}(B)$, então $T_{\frac{1}{2}}(1) V_{\delta}$ é uma pequena vizinhança de $\mathcal{A}_{h}$ em $X_{h}^{\eta}$ que contém $T_{\frac{1}{2}}(t+1) B=T_{\eta}(t+1) B$. Como $\mathcal{A}_{h}=T_{\frac{1}{2}}(1) \mathcal{A}_{h} \in X_{h^{\prime}}^{\eta}$ este deve ser o atrator de $T_{\eta}(t)$.

\subsection{Limitação uniforme dos atratores}

Com base no que foi desenvolvido na Seção 4 do Capítulo 1, obtemos, nesta seção, uma limitação uniforme da família de atratores $\left\{\mathcal{A}_{h} \mid\left\|h-i_{\Omega}\right\|_{C^{3}\left(\Omega, \mathbb{R}^{2}\right)}<\epsilon_{0}\right\}$.

Teorema 2.7.1 Suponha que as hipóteses do Teorema 2.6.5 sejam válidas. Então a família de atratores $\left\{\mathcal{A}_{h} \mid\left\|h-i_{\Omega}\right\|_{C^{2}\left(\Omega, \mathbb{R}^{2}\right)}<\epsilon_{0}\right\}$ é limitada uniformemente em $H^{1}(\Omega)$.

\section{Demonstração:}

Pelo Corolário 2.6.3, para $h$ suficientemente próxima de $i_{\Omega}$, o conjunto de pontos de equilíbrio $E_{h}$ de (2.5) está em uma bola aberta $B_{R}$ de raio $R$ em $H^{1}(\Omega), R$ independente de $h$.

Se $u \in \mathcal{A}_{h}$, pelo Teorema 3.8.5 em Hale (2000), existe $t_{u}$ tal que $u=T\left(t_{u}\right) u_{0}$ para algum $u_{0} \in B_{R}$.

Seja $V_{h}$ o funcional de Lyapunov de (2.5) dado por 2.15). Pelo Lema 2.5.3, temos

$$
V_{h}\left(u_{0}\right) \leq K_{1}\left\|u_{0}\right\|_{H^{1}(\Omega)}^{2}+K_{2} \leq K_{1} R^{2}+K_{2}
$$

Segue que

$$
V_{h}(u) \leq V_{h}\left(T\left(t_{u}\right) u_{0}\right) \leq V_{h}\left(u_{0}\right) \leq K_{1} R^{2}+K_{2}
$$

E,

$$
\|u\|_{H^{1}(\Omega)}^{2} \leq \frac{1}{K_{1}}\left(V_{h}(u)+K_{2}\right) \leq \frac{1}{K_{1}}\left(K_{1} R^{2}+2 K_{2}\right), \quad \forall u \in \mathcal{A}_{h} .
$$

Assim, se $\left\|h-i_{\Omega}\right\|_{C^{2}\left(\Omega, \mathbb{R}^{2}\right)}<\epsilon_{0}$, tal limitação é válida $\forall u \in \cup_{\left\|h-i_{\Omega}\right\|_{\mathcal{C}^{2}\left(\Omega, \mathbb{R}^{2}\right)}}<\epsilon_{0} \mathcal{A}_{h}$.

A seguir, obteremos uma limitação da família de atratores $\left\{\mathcal{A}_{h} \mid\left\|h-i_{\Omega}\right\|_{C^{3}\left(\Omega, \mathbb{R}^{2}\right)}<\epsilon_{0}\right\}$ em $L_{\infty}(\Omega)$. Para tanto, moldaremos a abordagem descrita na Seção 4 do Capítulo 1 ao contexto com o qual estamos trabalhando.

O primeiro passo é, a partir de uma limitação de $\mathcal{A}_{h}$ em $X_{h}^{\alpha}$ para algum $0 \leq \alpha<1$, limitar $\mathcal{A}_{h}$ 
em um espaço mais regular $X_{h}^{\alpha^{\prime}}, \alpha<\alpha^{\prime}<1$, onde tais constantes devem ser independentes de $h$, com o auxílio da Proposição 1.4.4. Precisaremos do seguinte Lema:

Lema 2.7.2 Para qualquer $0 \leq \alpha \leq 1$, seja $\|\cdot\|_{h, \alpha}$ a norma em $X_{h,-1 / 2}^{\alpha}=X_{h}^{\alpha-\frac{1}{2}} e\|\cdot\|_{\alpha}$ a norma em $X_{i_{\Omega},-1 / 2}^{\alpha}=X_{i_{\Omega}}^{\alpha-\frac{1}{2}}$. Então, $\|u\|_{h, \alpha} \leq K_{1}(h)\|u\|_{\alpha} \leq K_{2}(h)\|u\|_{h, \alpha}, \operatorname{com} K_{1}(h), K_{2}(h) \rightarrow 1$ quando $h \rightarrow i_{\Omega}$ uniformemente em $\alpha$.

\section{Demonstração:}

É conhecido que $-A_{h,-1 / 2}$ é positivo e autoadjunto. O resultado vem do Lema 2.2.3 e do Teorema 1.4.5.

Teorema 2.7.3 Para $\mathcal{A}_{h}$ tal que $\left\|h-i_{\Omega}\right\|_{C^{2}\left(\Omega, \mathbb{R}^{2}\right)}<\epsilon_{0}$, temos que $\mathcal{A}_{h}$ é limitado em $X_{h}^{\alpha^{\prime}}$ com $\frac{1}{2}<\alpha^{\prime}<1$. Além disso, tal limitação é independente de $h$.

\section{Demonstração:}

Para a primeira afirmação, basta combinarmos o Teorema 2.7.1 com a Proposição 1.4.4.

Como queremos uma limitação de $\mathcal{A}_{h}$ em $X_{h}^{\alpha}$ independente de $h$, precisamos examinar detalhadamente a seguinte desigualdade análoga a (1.14),

$$
\begin{aligned}
\left\|u\left(t_{0}+1 ; t_{0}, u_{0}\right)\right\|_{X_{h}^{\alpha^{\prime}}} & \leq\left\|\left(\left(-A_{h,-1 / 2}\right)_{\beta}\right)^{\alpha^{\prime}} e^{-\left(-A_{h,-1 / 2}\right)_{\beta}\left(t_{0}+1-t_{0}\right)_{0}}\right\| \\
& +\int_{t_{0}}^{t_{0}+1}\left\|\left(\left(-A_{h,-1 / 2}\right)_{\beta}\right)^{\alpha^{\prime}} e^{-\left(-A_{h,-1 / 2}\right)_{\beta}\left(t_{0}+1-s\right)}\left(H_{h,-1 / 2}\right)_{\beta}\left(u\left(s ; t_{0}, u_{0}\right)\right)\right\| d s \\
& \leq\left\|\left(\left(-A_{h,-1 / 2}\right)_{\beta}\right)^{\alpha^{\prime}-\frac{1}{2}} e^{-\left(-A_{h,-1 / 2}\right)_{\beta}\left(t_{0}+1-t_{0}\right)}\right\|\left\|u_{0}\right\|_{X_{h}^{\frac{1}{2}}} \\
& +\int_{t_{0}}^{t_{0}+1}\left\|\left(\left(-A_{h,-1 / 2}\right)_{\beta}\right)^{\alpha^{\prime}-\beta} e^{-\left(-A_{h,-1 / 2}\right)_{\beta}\left(t_{0}+1-s\right)}\right\|\left\|\left(H_{h,-1 / 2}\right)_{\beta}\left(u\left(s ; t_{0}, u_{0}\right)\right)\right\|_{X_{h}^{\beta}} d s \\
& \leq C_{h, \alpha^{\prime}-\frac{1}{2}}(1)^{-\left(\alpha^{\prime}-\frac{1}{2}\right)} e^{-\omega}\left\|u_{0}\right\|_{X_{h}^{\frac{1}{2}}} \\
& +\int_{t_{0}}^{t_{0}+1} C_{h, \alpha^{\prime}-\beta}\left(t_{0}+1-s\right)^{-\left(\alpha^{\prime}-\beta\right)} e^{-\omega\left(t_{0}+1-s\right)}\left\|\left(H_{h,-1 / 2}\right)_{\beta}\left(u\left(s ; t_{0}, u_{0}\right)\right)\right\|_{X_{h}^{\beta}} d s \\
& \leq C_{h, \alpha^{\prime}-\frac{1}{2}}\left(\frac{1}{K_{1}}\left(K_{1} R^{2}+2 K_{2}\right)\right)^{\frac{1}{2}}+C_{h, \alpha^{\prime}-\beta}\left\|\left(H_{h,-1 / 2}\right)_{\beta}\left(u\left(s ; t_{0}, u_{0}\right)\right)\right\|_{X_{h}^{\beta},}
\end{aligned}
$$

onde supusemos que $\mathfrak{R}\left(\sigma\left(\left(-A_{h,-1 / 2}\right)_{\beta}\right)\right)>\omega>0,-\frac{1}{2}<\beta<-\frac{1}{4}, C_{h, \alpha^{\prime}-\frac{1}{2}}$ e $C_{h, \alpha^{\prime}-\beta}$ são as constantes do Teorema 1.2.9 e aplicamos o Teorema 2.7.1.

Pela primeira parte do trabalho, ambas $C_{h, \alpha^{\prime}-\frac{1}{2}}, C_{h, \alpha^{\prime}-\beta}$ dependem essencialmente da $\left(C_{2}\right)_{h}$ para 
o operador $\left(-A_{h,-1 / 2}\right)_{\beta}$, ou seja, se a família $\left\{\left(-A_{h,-1 / 2}\right)_{\beta} \mid\left\|h-i_{\Omega}\right\|_{C^{2}\left(\Omega, \mathbb{R}^{2}\right)}<\epsilon_{0}\right\}$ admitir um setor e uma constante $M$ comuns a todos os seus elementos, temos que tais constantes não dependerão de $h$ e isso segue do Lema 2.2.3.

Com relação a limitação para $\left\|u_{0}\right\|_{X_{h}^{\frac{1}{2}}}$ obtida do Teorema 2.7.1, observamos que as constantes na expressão (2.23) podem ser tomadas independentes de $h$, pois $K_{1}, K_{2}$ e $R$ envolvem basicamente as não linearidades, $\max _{x \in \bar{\Omega}}\left\{\left|J_{h}\right|\right\}$ e $\lambda_{0}(h)$.

E embora os Lemas 2.4.2 e 2.4.4 nos garantam que $\left(H_{h,-1 / 2}\right)_{\beta}$ leva o limitado $\cup_{\left\|h-i_{\Omega}\right\|_{C^{2}\left(\Omega, \mathbb{R}^{2}\right)}<\epsilon_{0}} \mathcal{A}_{h}$ de $X_{h}^{\frac{1}{2}}$ em um limitado de $X_{h^{\prime}}^{\beta}$ notamos que as constantes relativas a tal limitação dependem de $h$. Agora, se soubermos que as normas nos espaços $X_{h,-1 / 2}^{\alpha}$ e $X_{i_{\Omega},-1 / 2}^{\alpha}$ são equivalentes para $0 \leq \alpha \leq 1$, essa questão estará resolvida. É o que nos diz o Lema 2.7.2.

Neste ponto, temos que para $\left\|h-i_{\Omega}\right\|_{C^{2}\left(\Omega, \mathbb{R}^{2}\right)}<\epsilon_{0},\|x\|_{X_{h}^{\alpha^{\prime}}}<S, \forall x \in \mathcal{A}_{h}$, onde $S$ independe de $h$, $\frac{1}{2}<\alpha^{\prime}<1$. A próxima etapa então é comparar as normas em $X_{h}^{\alpha^{\prime}}$ e $X^{\alpha^{\prime}}$.

Lema 2.7.4 Para $0 \leq \alpha \leq 1$, denotemos por $\|\cdot\|_{h, \alpha}$ a norma em $X_{h}^{\alpha}$ e $\|\cdot\|_{\alpha}$, a norma em $X^{\alpha}$. Então, $\|u\|_{h, \alpha} \leq K_{1}(h)\|u\|_{\alpha} \leq K_{2}(h)\|u\|_{h, \alpha}, \operatorname{com} K_{1}(h), K_{2}(h) \rightarrow 1$ quando $h \rightarrow i_{\Omega}$ em $C^{3}\left(\Omega, \mathbb{R}^{2}\right)$ uniformemente em $\alpha$.

Demonstração:

Segue do Lema 2.2.2 e do Teorema 1.4.5.

Teorema 2.7.5 $A \cup_{\left\|h-i_{\Omega}\right\|_{\mathcal{C}^{3}\left(\Omega, \mathbb{R}^{2}\right)}<\epsilon_{0}} \mathcal{A}_{h}$ está contida em uma bola de $X^{\alpha^{\prime}}$.

\section{Demonstração:}

Se $\left\|h-i_{\Omega}\right\|_{C^{3}\left(\Omega, \mathbb{R}^{2}\right)}<\epsilon_{0}$, usando o Teorema 2.7.3 e o Lema 2.7.4, para todo $x \in \mathcal{A}_{h}$,

$$
\|x\|_{X^{\alpha^{\prime}}} \leq K(h) S
$$

com $K(h) \rightarrow 1$ quando $h \rightarrow i_{\Omega}$ e $S$ é uma constante independente de $h$ que advém da limitação da família em $X_{h}^{\alpha^{\prime}}$.

Notemos que no último teorema, precisamos da convergência de $h$ para $i_{\Omega}$ na norma $C^{3}$, pois essa hipótese é necessária no Lema 2.7.4. Finalmente, 
Teorema 2.7.6 A família de atratores $\left\{\mathcal{A}_{h} \mid\left\|h-i_{\Omega}\right\|_{C^{3}\left(\Omega, \mathbb{R}^{2}\right)}<\epsilon_{0}\right\}$ para o problema (2.5) está limitada em $L_{\infty}(\Omega)$.

\section{Demonstração:}

De acordo com o Teorema 1.3.3, $X^{\alpha^{\prime}} \subset C^{v}$ visto que $\alpha^{\prime}>\frac{1}{2}$, ou seja, $X^{\alpha^{\prime}} \subset L_{\infty}(\Omega)$. E a conclusão segue do Teorema 2.7.5. 


\section{Referências Bibliográficas}

[1] Agmon, S., Douglis, A., \& Nirenberg, L. (1955) Estimates near the boundary for solutions of elliptic partial differential equations satisfying general boundary conditions. Communications on Pure and Applied Mathemathics, Vol. XII, 623-727.

[2] Arrieta, J. M., Carvalho, A. N. de, \& Rodríguez-Bernal, A. (2000). Attractors of parabolic problems with nonlinear boundary conditions. Uniform bounds. Communications in Partial Differential Equations, 25: 1-2, 1-37.

[3] Barbosa, P.S., Pereira, A. L., \& Pereira, M. C. (2016). Continuity of attractors for a family of $C^{1}$ perturbations of the square. Annali di Matematica. doi: 10.1007/s10231-016-0620-5

[4] Brezis, H. (2011). Functional analysis, Sobolev spaces and partial differential equations (Universitext ). Springer.

[5] Carvalho, A. N. de. (2001). Equações parabólicas semilineares. Recuperado de http://conteudo.icmc.usp.br/pessoas/andcarva/sg.pdf.

[6] Carvalho, A. N de. (2012). Sistemas dinâmicos não lineares. Recuperado de http://conteudo.icmc.usp.br/pessoas/andcarva/SDNL2012.pdf.

[7] Faustino, S. D., \& Nosaki, G. L. D. V. (2016). Operadores setoriais e potências fracionárias. (Trabalho apresentado na aula de Análise Funcional e Operadores Lineares Aplicados - IMEUSP).

[8] Hale, J. K. (2000). Asymptotic behavior of dissipative systems (Mathematical Surveys and Monographs, Vol. 25). Providence, RI: American Mathematical Society. 
[9] Henry, D. B. (1981). Geometric theory of semilinear parabolic equations (Lecture Notes in Mathematics, Vol. 840). Springer-Verlag.

[10] Henry, D. B.(2005). Perturbation of the boundary in boundary-value problems of partial differential equations (London Mathematical Society Lecture Note Series, Vol. 318). New York, NY: Cambridge University Press.

[11] Necas, J. (2012). Direct methods in the theory of elliptic equations (Springer Monographs in Mathematics). Springer.

[12] Oliveira, L. A. F. de, Pereira, A. L., \& Pereira, M. C. (2005). Continuity of attractors for a reaction-diffusion problem with respect to variations of the domain. Electronic Journal of Differential Equations, 100, 1-18.

[13] Pazy, A. (1983). Semigroups of linear operators and applications to partial differential equations (Applied Mathematical Sciences, Vol. 44). New York, NY: Springer-Verlag.

[14] Pereira, A. L., \& Pereira, M. C. (2007). Continuity of attractors for a reaction-diffusion problem with nonlinear boundary conditions with respect to variations of the domain. Journal of Differential Equations, 239, 343-370.

[15] Schmudgen, K. (2012). Unbounded self-adjoint operators on Hilbert spaces (Graduate Texts in Mathematics). Springer.

[16] Stein, E. M. (1970). Singular integrals and differentiability properties of functions(Princeton Mathematical Series). Princeton, NJ: Princeton University Press.

[17] Yagi, A. (2010). Abstract parabolic evolution equations and their applications (Springer Monographs in Mathematics). Springer. 